University of Louisville

ThinkIR: The University of Louisville's Institutional Repository

8-2013

\title{
6-phosphofructo-2-kinase inhibition induces autophagy as a survival mechanism.
}

Alden Klarer

University of Louisville

Follow this and additional works at: https://ir.library.louisville.edu/etd

\section{Recommended Citation}

Klarer, Alden, "6-phosphofructo-2-kinase inhibition induces autophagy as a survival mechanism." (2013). Electronic Theses and Dissertations. Paper 760.

https://doi.org/10.18297/etd/760

This Doctoral Dissertation is brought to you for free and open access by ThinkIR: The University of Louisville's Institutional Repository. It has been accepted for inclusion in Electronic Theses and Dissertations by an authorized administrator of ThinkIR: The University of Louisville's Institutional Repository. This title appears here courtesy of the author, who has retained all other copyrights. For more information, please contact thinkir@louisville.edu. 


\title{
6-PHOSPHOFRUCTO-2-KINASE INHIBITION INDUCES AUTOPHAGY AS A SURVIVAL MECHANISM
}

\author{
By \\ Alden Klarer \\ B.S., University of Kentucky, 2004 \\ M.S., University of Louisville, 2011 \\ A Dissertation \\ Submitted to the Faculty of the \\ School of Medicine of the University of Louisville \\ In Partial Fulfillment of the Requirements \\ for the Degree of \\ Doctor of Philosophy \\ Department of Biochemistry and Molecular Biology \\ University of Louisville \\ Louisville, Kentucky
}

August 2013 



\title{
6-PHOSPHOFRUCTO-2-KINASE INHIBITION INDUCES AUTOPHAGY AS A SURVIVAL MECHANISM
}

\author{
By \\ Alden Klarer \\ B.S., University of Kentucky, 2004 \\ M.S., University of Louisville, 2011
}

A Dissertation Approved on

May 21, 2013

by the following Dissertation Committee:

Dr. Sucheta Telang

Dr. Steven Ellis

Dr. Barbara Clark

Dr. Yong Li

Dr. Bradford Hil 


\section{ACKNOWLEDGEMENTS}

It is a good thing I am not getting an Emmy Award because they would kick me off the podium by the time I got done thanking everyone. The first are my mentors Dr. Sucheta Telang and Dr. Steve Ellis. My path through graduate school, like most everything in my life, was far from traditional. Without Dr. Ellis to guide me through not only the administrative stepping stones, but the intellectual ones as well, I would have likely given up. Dr. Telang so kindly welcomed me into her lab and allowed me the freedom to experiment with ideas that, while not always successful, taught me how to become a better scientist. I am so grateful to have her as a mentor, a friend, and my personal neonatologist. My other committee members, Dr. Barbara Clark, Dr. Yong Li, and Dr. Brad Hill were essential in providing direction for my project and insight for my dissertation. I am grateful to the faculty and staff in the Department of Biochemistry and Molecular Biology for their contributions to my scientific training. I am also appreciative of the M.D./Ph.D. Program including Dr. Donald Miller, Dr. Binks Wattenburg, and Victoria King, for allowing me to pursue my combined interests in research and medicine. I would also like to thank everyone if the lab and all of the people that I have collaborated with over the past four years including; Amy Clem, Dr. Julie O'Neill, Dr. Yoannis Imbert-Fernandez, Dr. Brain Clem, Amber McPherson, Jennifer Clark, Miriam Reynolds, Dr. Abdullah 
Yalcin, Dr. John Eaton, Dr. Bob Mitchell, Dr. Chi Li, Dr. Erin Brock, Lilibeth Lanceta, Dr. Deanna Siow, Tom Burke, and Dr. Jay Stallons Of course I would have never come this far without my entire family including my grandparents, aunts, uncles and cousins, whose love and support were indispensable in shaping who I am. My parents instilled in me the value of having great pride in what you do. My dad showed me how to have the confidence to think independently, to set realistic goals, to embrace my wild-side, and the importance of starting off the day with a good breakfast even if it is spaghetti. My mom taught me how to be cautiously adventurous, letting me have the freedom to forge my own path while giving me the security of knowing that I would be loved regardless of my successes or failures. The significance of this support was exponentially magnified over the past year as she extended herself even further by forming the "Oma Loving and Learning Center for Calvin", for which I will never fully be able to repay her. My brother's passionate intensity and great talent continues to be an inspiration for me. I also want to thank Madeleine and Calvin for fueling my maturation to adulthood and helping me realize what is really important in life. Lastly, I am indebted to my husband, Jason. I am in perpetual admiration for his determination to make his work in research and his practice lead to significant clinical outcomes that lead to real improvements in people's lives. We have driven over some rough road at times but our lasting endurance is evidence of our love and commitment to each other's happiness. 


\title{
ABSTRACT
}

\section{6-PHOSPHOFRUCTO-2-KINASE INHIBITION INDUCES AUTOPHAGY AS A SURVIVAL MECHANISM}

\author{
Alden Klarer
}

May 21, 2013

Altered metabolism has long been recognized as a defining characteristic of tumor cells. The increased glycolytic phenotype, an observation credited to Otto Warburg in 1956, is almost universal for cancer cells, making metabolic enzymes attractive targets for cancer therapy. However, anti-metabolic drugs, thus far, have not lived up to expectations as stand-alone agents to treat this disease. Unlike glycolytic enzymes that directly catabolize glucose to pyruvate, the family of 6-phosphofructo-2-kinase/fructose-2,6-bisphosphatases (PFKFBs) control the conversion of fructose-6-phosphate to and from fructose-2,6bisphosphate, a key regulator of the glycolytic enzyme phosphofructokinase-1 (PFK-1). One isoform, PFKFB3, has been shown to be highly expressed by human cancer cells and a specific PFKFB3 small molecule inhibitor, (3-(3- 
pyridinyl)-1-(4-pyridinyl)-2-propen-1-one [3PO] is currently being developed for clinical use. However, the effectiveness of current chemotherapeutics is limited by the development of drug resistance, which is especially problematic for antimetabolic drugs where the starvation state activates a variety of survival mechanisms within the cell that contribute to resistance. One such mechanism used by all cells to survive nutrient-poor conditions is the activation of autophagy, the process of cellular self-catabolism. Autophagic induction allows for the continued generation of biosynthetic intermediates that can be used for energy generation and critical metabolic processes while also ensuring prompt disposal of damaged and malfunctioning organelles before they cause cellular harm. We hypothesized that the functional starvation induced by inhibition of PFKFB3 in tumor cells might induce autophagy as a pro-survival mechanism and that the combination of drugs targeting PFKFB3 with pharmacologic inhibitors of autophagy could increase anti-tumor effects. This hypothesis has been tested and our data reveal that knockdown or inhibition of PFKFB3 results in autophagic induction. This induction appears to serve as a survival mechanism as evidenced by furthered cell death upon the addition of inhibitors of autophagy. 


\section{TABLE OF CONTENTS}

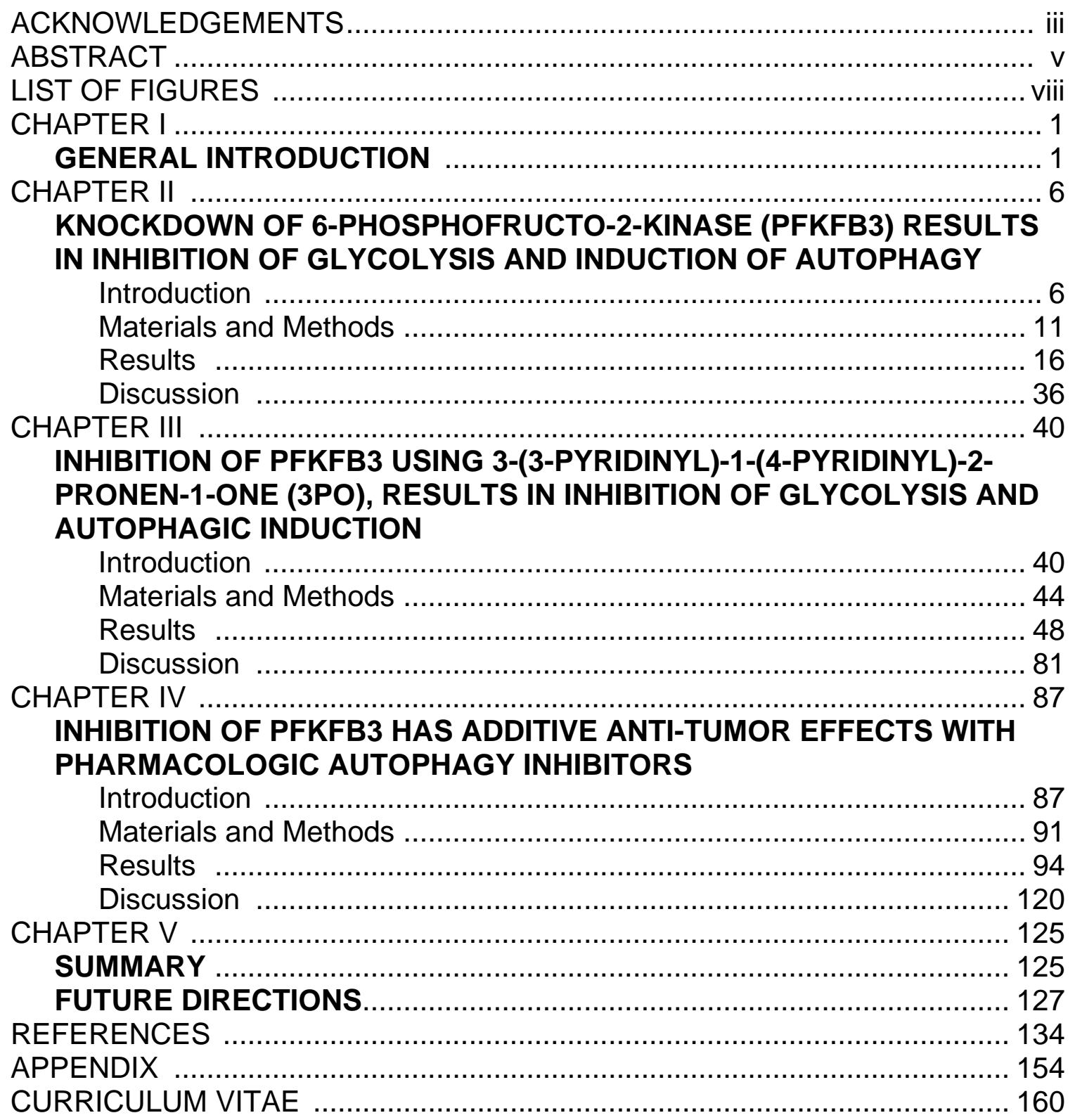




\section{LIST OF FIGURES}

PAGE

Figure 1: Treatment of cells with siRNA directed against PFKFB3 mRNA results in decreased steady-state levels of PFKFB3 protein 17

Figure 2: Knockdown of PFKFB3 reduces fructose 2,6 bisphosphate (F2,6BP) levels 19

Figure 3: $2-\left[1-{ }^{14} \mathrm{C}\right]-$ deoxy-D-glucose uptake is reduced by PFKFB3 knockdown 21

Figure 4: Protein markers of autophagy, LC3-II and p62, are altered after knockdown of PFKFB3

Figure 5: Acridine orange immunofluorescence is increased by PFKFB3 knockdown

Figure 6: Autophagic structures are visible by electron microscopy after knockdown of PFKFB3 29

Figure 7: Protein markers downstream of mTOR are decreased by PFKFB3 knockdown 32

Figure 8: PFKFB3 knockdown reduces ATP levels 34

Figure 9. 3-(3-pyridinyl)-1-(4-pyridinyl)-2-propen-1-one (3PO), was designed to inhibit PFKFB3 activity 42

Figure 10: Small molecule inhibition of PFKFB3 reduces fructose 2,6,bisphosphate (F2,6BP) levels 49

Figure 11: A small molecule inhibitor of PFKFB3, 3PO, reduces $2-\left[1-{ }^{14} \mathrm{C}\right]-$ deoxy-D-glucose uptake.... 51

Figure 12: Markers of autophagy, LC3-II and p62, are altered by $3 \mathrm{PO}$ 54

Figure 13: $3 \mathrm{PO}$ increases acridine orange immunofluorescence 56 
Figure 14: Inhibition of PFKFB3 results in autophagic structures visualized by electron microscopy

Figure 15: $3 \mathrm{PO}$ treatment results in decreased expression of downstream mTOR effectors 61

Figure 16: ATP levels are decreased by treatment with 3PO ........................63 63

Figure 17: 3PO treatment causes a reduction in glutathione ......................66

Figure 18: Reactive oxygen species are increased by treatment with $3 \mathrm{PO}$....68

Figure 19: $\mathrm{N}$-acetylcysteine prevents $3 \mathrm{PO}$ induced reduction in glutathione levels

Figure 20: Reactive oxygen species production by $3 \mathrm{PO}$ is rescued by $\mathrm{N}$ acetylcysteine ….................................................................................. 73

Figure 21: LC3-II induction by 3PO is blocked by $\mathrm{N}$-acetylcysteine ................ 75

Figure 22: 3PO-induced acridine orange immunofluorescence is blocked by $\mathrm{N}$ acetylcysteine .................................................................................. 77

Figure 23: $\mathrm{N}$-acetylcysteine rescues cells from death after treatment with 3PO79

Figure 24: Synergistic increase in cell death when $3 \mathrm{PO}$ is combined with the autophagy inhibitor chloroquine 96

Figure 25: The autophagy inhibitor, 3-methyladenine, increased cell death when combined with $3 \mathrm{PO}$ 98

Figure 26. The combination of Spautin-1 and 3PO increased cell death ...... 100

Figure 27. The 3PO derivative PF158 causes increased cell death when combined with chloroquine 102

Figure 28. Cell death by PF-15, a derivative of $3 \mathrm{PO}$, is increased when combined with chloroquine 104

Figure 29. Cell death due to knockdown of PFKFB3 is increased when combined with the autophagy inhibitor chloroquine 106

Figure 30: Autophagic protein marker LC3-II is increased in Lewis lung carcinoma cells exposed to $3 \mathrm{PO}$ 109 
Figure 31: Lewis lung carcinoma cells treated with 3PO have increased acridine

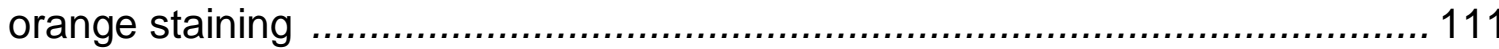

Figure 32. The combination of $3 \mathrm{PO}$ and chloroquine has synergistic effects on cell death in Lewis lung carcinoma cells 113

Figure 33: Combination therapy using 3PO and chloroquine reduces tumor growth is a Lewis lung carcinoma tumor model in vivo 116

Figure 34: Immunohistochemistry of excised tumors from 3PO + chloroquine treated animals have increased cleaved-caspase 3 staining..... 118

Figure 35: Inhibition of glycolysis causes induction of autophagy for survival 


\section{CHAPTER I}

\section{GENERAL INTRODUCTION}

\section{Cancer cells have altered cellular metabolism.}

The metabolic alterations characteristic of tumor cells have now reached hallmark status [1]. Increased glucose uptake and utilization, first described by Warburg in 1956, provides biosynthetic precursors for these rapidly dividing cells and also may be a means by which cancer cells compensate for reduced ATP production [2, 3]. This counterintuitive replacement of a more efficient system, oxidative respiration, that generates $36 \mathrm{~mol}$ of ATP per mol of glucose, with glycolysis which only produces 2 ATP, to generate increased energy needed by highly proliferative tumor cells has been rationalized by the rapid rates at which glucose can be metabolized through glycolysis [4]. There are a number of theories pertaining to why tumor cells take up and utilize more glucose than normal cells. Some have proposed that mitochondrial defects result in the preferential use of glycolysis; however, support for this view is limited [5-7]. Others speculate that hypoxia encountered within the tumor microenvironment due to outgrowing its blood supply, results in the shift to glycolysis [8]. Whether due to hypoxia, or to other causes, it is clear that changes in glucose uptake or glycolytic flux can result from changes in the 
expression or activity of a number of key proteins involved in the glycolytic pathway including glucose transporter-1, hexokinase, pyruvate kinase, and a family of enzymes involved in the regulation of glycolysis, the PFKFBs [9-16].

\section{PFKFB3 can regulate glycolysis by controlling levels of F2,6BP}

Bifunctional 6-phosphofructo-2-kinase/fructose-2,6-bisphosphatases (PFKFBs) regulate glycolytic flux by controlling the steady state concentration of fructose 2,6 bisphosphate (F2,6BP), a potent allosteric regulator of PFK-1[17]. The PFKFB family consists of 4 isoforms of which PFKFB3 is of particular interest in tumor cells [18]. PFKFB3 mRNA and protein are increased in tumors when compared to normal tissue $[19,20]$. This increase is mediated in part through repeating AUUUA elements in the 3'-UTR of its mRNA which are present in the mRNAs of several proto-oncogenes and have been shown to increase translational activity [21]. Additionally, PFKFB3 has a kinase: phosphatase ratio of $740: 1$, due to the lack of regulatory residues present on other isoforms [22]. The result is a considerable increase in $\mathrm{F} 2,6 \mathrm{BP}$ and subsequent upregulation of glycolysis following increased PFKFB3 expression [23-25]. Knockdown of PFKFB3 using siRNA decreases glycolysis, cell proliferation and inhibits anchorage-independent growth, making this enzyme a promising target for anticancer therapy [26]. Despite the development of numerous anti-metabolic agents to treat cancer, these drugs have not seen the clinical success anticipated based on their pre-clinical profiles and their presumed tumor-specific activity. [27-32]. Interestingly, in a clinical trial testing 2-deoxyglucose, a competitive 
inhibitor of glucose metabolism [33] in prostate cancer patients, an autophagic resistance, indicated by decreased p62 expression, was observed in the majority of the patients treated [34]. Recently, the combination of autophagy inhibitors with compounds that induce metabolic stress has drawn great attention and chloroquine, a drug that has been shown to inhibit autophagy, is now being used alone and in combination with other chemotherapeutics in human cancer trials [35-37]

\section{Anti-metabolic targeting can mimic a low nutrient environment and lead to activation of autophagy}

Cells in low nutrient environments, such as those with reduced amino acids or glucose, activate the cellular self-digestion process of autophagy [3840]. While this process occurs at a basal level within cells playing a complementary role with the proteasome to help clear larger and more abundant material, the induction of autophagy can be triggered by stressful stimuli such as hypoxia or nutrient deprivation. Under these conditions, autophagy is a means by which cells are able to degrade cellular components to provide biosynthetic precursors which can be used for anabolic processes and energy production [4144]. Targeting cancer cell metabolism is a promising strategy for anti-tumor therapy due to selectivity for the more metabolically active tumor cells. However, as with many other chemotherapeutic agents, cancer cells have developed resistance mechanisms in response to treatment with anti-metabolic drugs. Amongst these resistance mechanisms, the induction of autophagy may play an 
especially critical role in conferring resistance to anti-metabolic drugs since they induce states that mimic low nutrient environments that can stimulate autophagy. For example, 2-deoxy-glucose has been shown to induce autophagy both in vitro and in vivo as part of a phase I clinical trial for prostate cancer [34, 45, 46].

\section{Autophagy may serve to protect cells from metabolic stress}

Although autophagy has been implicated as either a mechanism for adaptive cell survival or for cell death, the process can serve either function depending on the stimulus, the timing, and the degree of activation. There are numerous examples of the pro-survival role of autophagy in cells. Potentially hazardous damaged mitochondria are routinely degraded by autophagic selfdigestion [47, 48]. Amino acids can stimulate the TCA cycle by increasing capacity through anaplerotic reactions that produce compounds like $\alpha$ ketoglutarate. Amino acids and fatty acids also produce acetyl-CoA which, as a substrate for the TCA cycle, is converted to carbon dioxide and water fueling cellular energetic processes [41-44]. Autophagy has also been shown to promote cellular survival in tumor cells treated with chemotherapeutic agents, e.g. etoposide, temozolomide, trastuzumab and imatinib [49-52]. Due to this demonstrated function of autophagy in protecting cells experiencing stress, it is reasonable to postulate that this process may protect cells that are confronted with the starvation condition created by metabolic inhibitors. 


\section{Co-targeting PFKFB3 and the autophagic pathway may result in increased anti-cancer effects}

Molecular modeling has allowed for the development of novel small molecule inhibitors that are able to target PFKFB3 enzyme activity. One such inhibitor, 3-(3-pyridinyl)-1-(4-pyridinyl)-2-propen-1-one (3PO), has been shown to suppress glycolysis flux to lactate, decrease glucose uptake and attenuate the proliferation of human cancer cell lines in vitro [53]. Similar inhibition was also observed in vivo, and, although tumor growth was decreased by treatment with 3PO, it was not completely suppressed [53]. We postulated that the metabolic stress caused by PFKFB3 inhibition might activate autophagy as a survival pathway that could confer resistance to $3 \mathrm{PO}$. Chloroquine (CQ), an anti-malarial agent that is relatively well tolerated and has been used in humans since the 1940's has been shown to inhibit autophagy and potentiate cancer cell death and is now being added to a number of other drugs as a part of several human cancer clinical trials [54-59]. We hypothesized that the combination of the PFKFB3 inhibitor 3PO with the autophagy inhibitor CQ might lead to a significant improvement in anti-cancer effect of $3 \mathrm{PO}$ in vitro and that this combination might also increase efficacy of 3PO as an anti-tumor agent in vivo which could have major implications for clinical trials using PFKFB3 inhibitors. 


\section{CHAPTER II}

\section{KNOCKDOWN OF 6-PHOSPHOFRUCTO-2-KINASE (PFKFB3) RESULTS IN INHIBITION OF GLYCOLYSIS AND INDUCTION OF AUTOPHAGY}

\section{Introduction}

Tumor cell dependence on increased glucose uptake and glycolysis allows for the increased production of ATP, nucleic acids, amino acids and fatty acids required for proliferation [60]. Glycolytic flux is controlled through the irreversible, rate-limiting enzyme, PFK-1. This enzyme is allosterically inhibited by ATP, which reduces flux when cellular ATP production exceeds utilization [61]. When energy reserves are reduced, AMP activates PFK-1. However, fructose 2,6 bisphosphate (F2,6BP), is also a potent activator of PFK-1 [17]. The family of 6-phosphofructo-2-kinase/fructose-2,6-biphosphatases (PFKFBs) is responsible for establishing the steady state levels of cellular F2,6BP. 
The PFKFB family consists of four members, (PFKFB1-4), which act to regulate the concentration of F2,6BP in cells [62-64]. The PFKFB3 isoform, with a kinase: phosphatase ratio of $740: 1$, due to the absence critical residues required for regulating kinase and phosphatase activity [65] has been shown to be expressed at higher levels in tumor cells [20]. This upregulation is mediated in part through an AUUUA-rich region in the 3' untranslated region of its mRNA which controls mRNA stability and translational activity $[23,65,66]$. The PFKFB3 isoform also contains a hypoxia responsive element making it a direct transcriptional target of HIF-1 $\alpha$ [22, 67-69]. Finally, PFKFB3 is activated by mitogenic signals and is stabilized after the loss of PTEN [23, 70-75]. These data indicate that the PFKFB3 isoform may play a role in the increased glycolytic phenotype of tumor cells relative to normal cells, which has led to the interest in targeting PFKFB3 as an anti-cancer strategy. Additionally, previous studies have shown that knockdown of PFKFB3 reduces glycolytic flux and has antiproliferative effects in tumors cells [26].

The interest in PFKFB3 as an anti-tumor target has resulted in the development of a number of small molecule inhibitors against the enzyme [53, 76] of which some are in the process of entering Phase I clinical trials as anticancer therapeutics.

Chemotherapies, even those with extremely promising results in vitro, have long been plagued by resistance when administered to cancer patients. Amongst the large number of resistance mechanisms, autophagy has been shown to be activated by a large number of chemotherapeutic agents as a pro- 
survival pathway and is now being studied as a potential target for both single and combination cancer therapy trials $[50,52,77,78]$.

Autophagy, a term first coined by Christian de Duve in 1963 is the evolutionarily conserved process of cellular self-catabolism [79]. Of the three types of autophagy, macroautophagy, microautophagy, and chaperone-mediated autophagy, macroautophagy is the most well-characterized and is referred to as autophagy in this dissertation. Autophagy is normally active at low levels in most cells and functions to remove long-lived proteins and organelles. This process has also been observed to protect cells by removing intracellular pathogens, potentially harmful damaged mitochondria and in generating metabolic substrates during nutrient deprivation [41-44, 80]. The process of autophagy involves the initial formation of a double-membraned vesicle, thought to originate from de novo synthesis as well as sources of cellular membrane such as the plasma membrane, the Golgi complex, endoplasmic reticulum or the mitochondrial membrane [81-87]. As maturation of this membrane continues, LC3-I is modified by the addition of phosphatidylethanolamine to form LC3-II which is incorporated into the growing membrane as a scaffolding protein [88]. Cargo is recruited to this forming autophagosome by p62, an adapter molecule with ubiquitin-binding domains that recognize poly-ubiquitinated organelles, or proteins [89]. Ultimately, autophagosomes fuse with lysosomes where cargo is degraded [89]. Autophagy normally functions at basal levels in most cells can be induced by a variety of stressful stimuli such as hypoxia or glucose deprivation, where it is presumed to promote survival [90]. 
Regulation of autophagy is mainly controlled through inhibition by the mammalian target of rapamycin (mTOR) pathway. In response to rapamycin, a compound isolated from soil-dwelling bacterial and originally used as an antimicrobial agent, mTOR activity is suppressed [91]. However, mTOR is now known to be regulated by other stimuli, especially signals associated with cellular energy status. When nutrients are plentiful, mTOR phosphorylates and activates ribosomal S6 kinases resulting in increased protein translation [92, 93]. At the same time, mTOR blocks autophagy by inhibition of a critical component in the autophagic pathway, Ulk1[94]. Upon nutrient deprivation, such as that experienced by glucose withdrawal, the AMP:ATP ratio is altered and AMP, and more recently revealed, ADP, binds to cellular energy sensor AMP-activated protein kinase (AMPK) causing a conformational change that favors the activation via phosphorylation at Thr 172 by upstream kinase LKB1 [95-98]. Upon phosphorylation, AMPK can inhibit mTOR activity by phosphorylation of TSC2 which suppresses Rheb, an mTOR activator [99, 100]. AMPK can also directly phosphorylate ULK1 to induce autophagy $[99,101,102]$. In this way, nutrient deprivation can signal both the inhibition of protein translation and the activation of the catabolic process of autophagy [103].

Previous studies have shown that loss of PFKFB3 expression or inhibition of its enzyme activity result in decreased glucose uptake, effectively starving the cells. Cellular responses to metabolic stress during starvation are varied and depend on numerous factors including but not limited to; cell type, the proliferative state of the cell and the extent and time of starvation. The induction 
of autophagy is one response mechanism that has been shown to increase cell survival during times of metabolic stress by allowing for the production of nutrients and sustained metabolism [41-44].

Based on the known metabolic effects of PFKFB3 targeting, we sought to determine the effects that knockdown of PFKFB3 would have on the activation of autophagy. In the current chapter, we find that knockdown of PFKFB3 results in an increase in autophagy and that this increase is likely mediated through the mTOR signaling pathway. 


\section{Materials and Methods}

\section{Cell Culture}

Human colorectal carcinoma cells (HCT116) obtained from American Type Culture Collection (Manassas, VA) and were cultured with McCoy's 5A medium (Gibco, Grand Island, NY) supplemented with 10\% calf serum and $50 \mathrm{ug} / \mathrm{mL}$ gentamicin. Cells were incubated at $37^{\circ} \mathrm{C}$ with $5 \% \mathrm{CO} 2$.

\section{Transfection}

HCT-116 cells were plated at 100,000 cells/well of a 6 -well dish in $2.5 \mathrm{ml}$ complete medium. $24 \mathrm{~h}$ after seeding, cells were transfected with either control siRNA (Stealth Negative Control Medium GC Duplex), or PFKFB3 siRNA (PFKFB3HSS107860; 5' UUCAUCAGGUAGUACACGCGGC 3', both from Invitrogen, Grand Island, NY). OptiMEM (Invitrogen, Grand Island, NY) with 1\% Lipofectamine RNAiMAX (Invitrogen, Grand Island, NY) was incubated at RT for

5 minutes. siRNA was added to the Lipofectamine mixture and incubated for 20 minutes at room temperature. The mixture was added to a single well of the 6well plate for a total volume of $3 \mathrm{ml}$ and a final siRNA concentration of $10 \mathrm{nM}$. Cells were incubated at $37^{\circ} \mathrm{C}$ for 48 hours before harvest. Samples in which 
bafilomycin A1 was used were treated with $1 \mathrm{nM}$ bafilomycin A1 (Sigma, St. Louis, MO) for 24 hours prior to harvest.

\section{Protein Extraction}

Cells were washed with PBS then lifted in 0.25\% Trypsin (Gibco, Grand Island, NY) and pelleted by centrifugation. Pellets were lysed in protein lysis buffer (Thermo, Rockford, IL) supplemented with protease and phosphatase inhibitors (Sigma, St. Louis, MO). Samples were homogenized by passing repeatedly through a $28 \quad 1 / 2$ gauge needle and then incubated on ice for 20 minutes before centrifugation at $2,000 \mathrm{~g}$ for 5 minutes at $4{ }^{\circ} \mathrm{C}$ and collection of supernatants. Protein concentration was determined using bicinchoninic acid assay (Thermo, Rockford, IL).

\section{Immunoblotting}

Equal amounts of protein were added to loading buffer (BioRad, Hercules, CA) containing $10 \mathrm{ul} / \mathrm{ml} \beta$ mercaptoethanol and heated to $98^{\circ} \mathrm{C}$ for 5 minutes and then loaded onto a 4-12\% gradient SDS-polyacrylamide gel (BioRad, Hercules, CA) and run for 60 minutes at 130 volts. Protein was transferred to a nitrocellulose membrane over 1 hour at $400 \mathrm{~mA}$ and then blocked in 5\% non-fat milk for 1 hour before incubation with primary antibodies. Antibodies against LC3, p62, pAMPK, AMPK, p-p70S6K, p70S6K, pS6, S6 (Cell Signaling, Danvers, MA), PFKFB3, Tel2 (Protein Tech, Chicago, IL), $\beta$ actin (Sigma, St. Louis, MO) were diluted 1:1000 and were incubated overnight at $4{ }^{\circ} \mathrm{C}$, with the exception of 
p62 and $\beta$ actin $A b$ that were incubated at room temperature for 1 hour. Membranes were washed for 30 minutes in Tris-Buffered Saline with Tween-20 (50 mM Tris- $\mathrm{HCl}, \mathrm{pH} 7.4,150 \mathrm{mM} \mathrm{NaCl}, 0.1 \%$ Tween 20) before addition of secondary antibodies (anti-mouse or anti-rabbit, diluted 1:10,000 in TBS-T, (Sigma, St. Louis, MO). ECL Western Blotting Detection Kit (Amersham/GE Pittsburgh, PA) was used to develop membranes. Quantitative densitometry was performed using Image $\mathrm{J}(\mathrm{NIH})$.

\section{F2,6BP Assay}

Intracellular fructose 2,6 bisphosphate (F2,6BP) levels were determined using a method previously described [104]. Briefly, HCT-116 cells were harvested 48 hours after transfection and centrifuged at $200 \times \mathrm{g}$. The pellets were resuspended in $50 \mathrm{mM}$ Tris acetate $(\mathrm{pH} 8.0)$ and $100 \mathrm{mM} \mathrm{NaOH}$ then incubated at $80{ }^{\circ} \mathrm{C}$ for five minutes and then placed on ice. Extracts were neutralized to $\mathrm{pH} 7.2$ with $1 \mathrm{M}$ acetic acid and $1 \mathrm{M}$ Hepes and then incubated at $25{ }^{\circ} \mathrm{C}$ for $2 \mathrm{~min}$ in $50 \mathrm{mM}$ Tris, $2 \mathrm{mM}$ Mg2+, $1 \mathrm{mM}$ F6P, 0.15mM NAD, 10u/l PPidependent PFK1, $0.45 \mathrm{kU} / \mathrm{l}$ aldolase, $5 \mathrm{kU} / \mathrm{l}$ triosephosphate isomerase, and 1.7 $\mathrm{kl} / \mathrm{l}$ glycerol-3-phosphate dehydrogenase. $0.5 \mathrm{mM}$ pyrophosphate was added and the rate of change in absorbance $(O D=339 \mathrm{~nm})$ per min over five minutes was determined. A calibration curve using 0.1 to 1 pmol of F2,6BP (Sigma, St. Louis, $\mathrm{MO}$ ) was used to calculate $\mathrm{F} 2,6 \mathrm{BP}$ which was then normalized to total protein. 


\section{Glucose Uptake Assay}

HCT-116 cells were plated at 100,000 cells in each well of a 6 -well dish. Cells were transfected with either control siRNA or siRNA directed against PFKFB3. 48 hours post-transfection, cells were washed with PBS and media was replaced with glucose-free RPMI 1640 for 30 minutes. 2-[1- $\left.{ }^{14} \mathrm{C}\right]$-deoxy-Dglucose (Perkin Elmer, Waltham, MA) was added for 30 minutes. Cells were then washed three times with ice-cold RPMI 1640 containing no glucose. Cells were lysed with $0.1 \%$ SDS. Scintillation counts (counts $/ \mathrm{min}$ ) were measured on a portion of lysate and normalized to protein concentration using the remainder of the lysate. Data are represented as mean \pm SD from duplicate samples.

\section{Acridine Orange Immunofluorescence}

After 48 hours of transfection, HCT-116 cells were washed with PBS and then stained with $0.01 \mathrm{mg} / \mathrm{ml}$ acridine orange in PBS for 15 minutes at $37{ }^{\circ} \mathrm{C}$. Cells were washed twice with PBS then harvested for study by microscopy or flow cytometry. For immunofluorescent examination and imaging, cells were viewed using an EVOSfl fluorescent microscope (AMG, Grand Island, NY). Acridine orange was visualized using an overlay of GFP and RFP filters. For flow cytometry, green (510-530nm) and red (650nm) fluorescence emission from 10,000 cells illuminated with blue $(488 \mathrm{~nm})$ excitation light was measured (BD FACSCalibur, San Jose, CA). FlowJo software (TREE STAR Inc, San Carlos, CA) was used to calculate the red: green fluorescence ratio. 


\section{Electron Microscopy}

HCT-116 cells were prepared for electron microscopy 48 hours posttransfection. Cells were washed twice with PBS and fixed in cold glutaraldehyde ( $3 \%$ in $0.1 \mathrm{M}$ cacodylate buffer, $\mathrm{pH} 7.4$ ) for 30 minutes. Samples were post fixed in $\mathrm{OsO} 4$ and $100 \mathrm{~nm}$ sections were taken and stained with uranyl/lead citrate and viewed using a transmission electron microscope (Phillips CM12). Methodology and identification of autophagic structures was based on established criteria and previous studies [105-107].

\section{$\underline{\text { ATP Determination }}$}

ATP levels were determined using a bioluminescence assay (Invitrogen, Grand Island, NY) following established protocols from suppliers. Briefly, cells were lysed on cultured plates using a passive lysis buffer and snap frozen in liquid nitrogen then thawed at $37^{\circ} \mathrm{C}$ and spun at $1200 \times \mathrm{g}$ for 30 seconds and $4^{\circ} \mathrm{C}$ to clear the lysates. Lysate was added to a prepared reaction solution containing reaction buffer, DTT, d-luciferin and firefly luciferase and luminescence was read using a luminometer (Biotek, Winooski, VT). ATP was determined based on a standard curve using 1-500nM ATP and was calculated relative to protein concentration. 


\section{Results}

\section{Knockdown of PFKFB3 results in decreased glycolytic flux}

In order to reduce PFKFB3 levels, a siRNA directed against PFKFB3 was transfected into human colorectal carcinoma cells (HCT-116). Protein expression, assessed by Western blotting, was inhibited by $>90 \%$ at 48 hours by the PFKFB3-specific siRNA relative to cells transfected with the negative control ( $p$ value $<0.05)$ (Figure 1). After confirmation of siRNA-mediated knockdown, the product of the enzyme, fructose 2,6 bisphosphate $(\mathrm{F} 2,6 \mathrm{BP})$ was measured using an enzyme-coupled assay. Knockdown of PFKFB3 resulted in a $60 \%$ reduction in $\mathrm{F} 2,6 \mathrm{BP}$ at 48 hours after transfection ( $p$ value $<0.05$ ) (Figure 2). Blocking the production of F2,6BP leads to reduced activity of PFK-1 [23]. Glucose uptake was used as an indirect measure of PFK-1 activity as loss of PFK-1 activity results in the intracellular accumulation of glucose and its derivatives reducing further glucose uptake. To measure glucose uptake 2-[1${ }^{14} \mathrm{C}$ ]-deoxy-D-glucose (2DG) was added to cells transfected with either a negative control siRNA or a siRNA directed against PFKFB3. 2DG uptake was reduced by more than $50 \%$ ( $p$ value $<0.05$ ) after 48 hours in cells with reduced PFKFB3 expression relative to the negative control (Figure 3). 
Figure 1. Treatment of cells with siRNA directed against PFKFB3 mRNA results in decreased steady-state levels of PFKFB3 protein. HCT-116 cells were transfected with either a control siRNA (ctrl) or 10nM of a siRNA directed against PFKFB3 (PFKFB3). Total protein was harvested 48 hours posttransfection and protein levels relative to $\beta$ actin were determined by Western blotting. Densitometry data are presented as the mean fold change \pm SD from three experiments. $(p$ value $<0.05)$ 
Figure 1

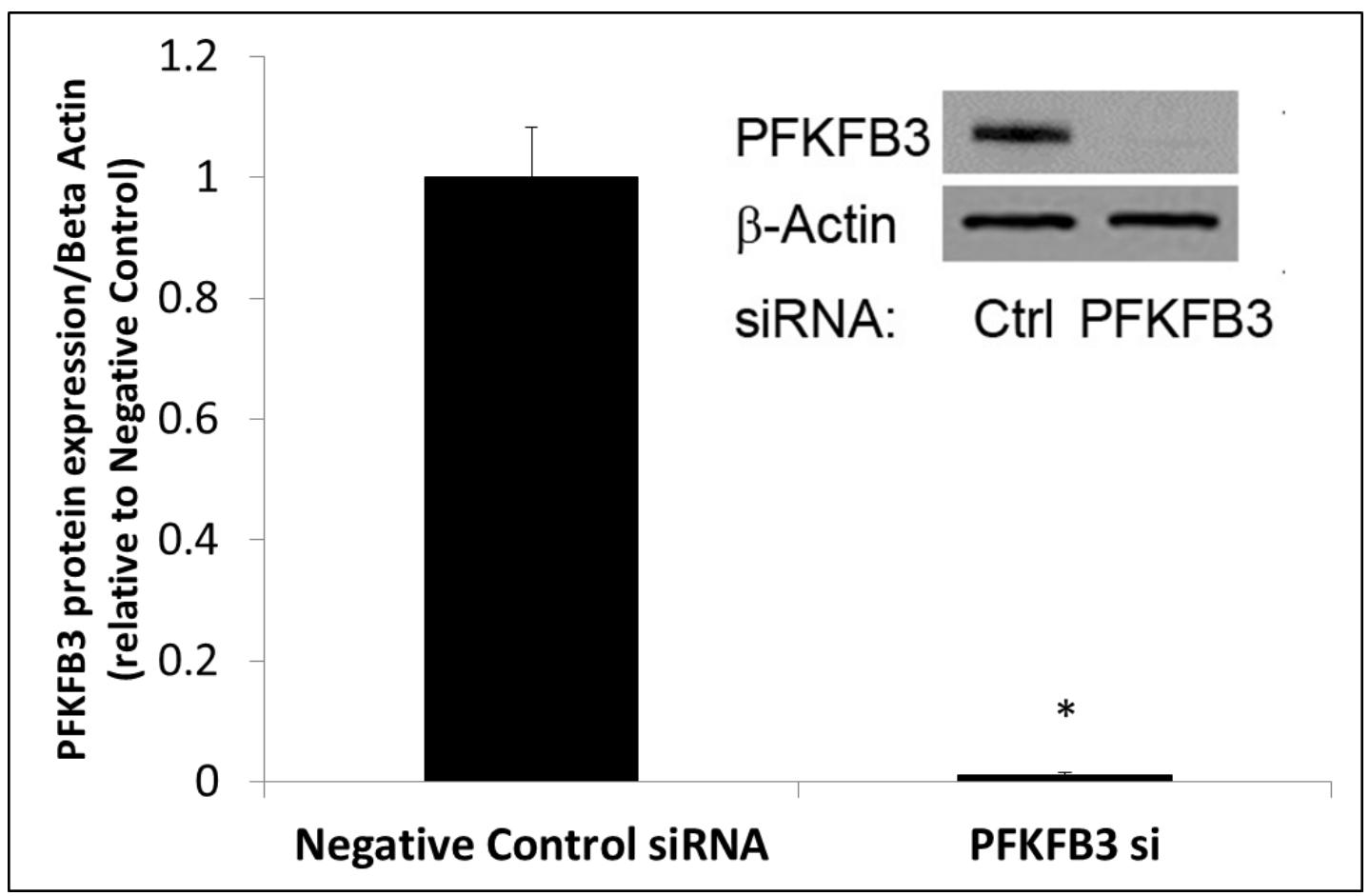


Figure 2. Knockdown of PFKFB3 reduces fructose 2,6 bisphosphate (F2,6BP) levels. HCT-116 cells were transfected with either a control siRNA (ctrl) or 10nM of a siRNA directed against PFKFB3 (PFKFB3). Fructose 2,6 bisphosphate levels were determined using an enzyme-coupled assay. Data are presented as the mean $\mathrm{pmol} / \mathrm{mg}$ protein \pm SD from three experiments. ( $p$ value $<$ 0.05). 
Figure 2.

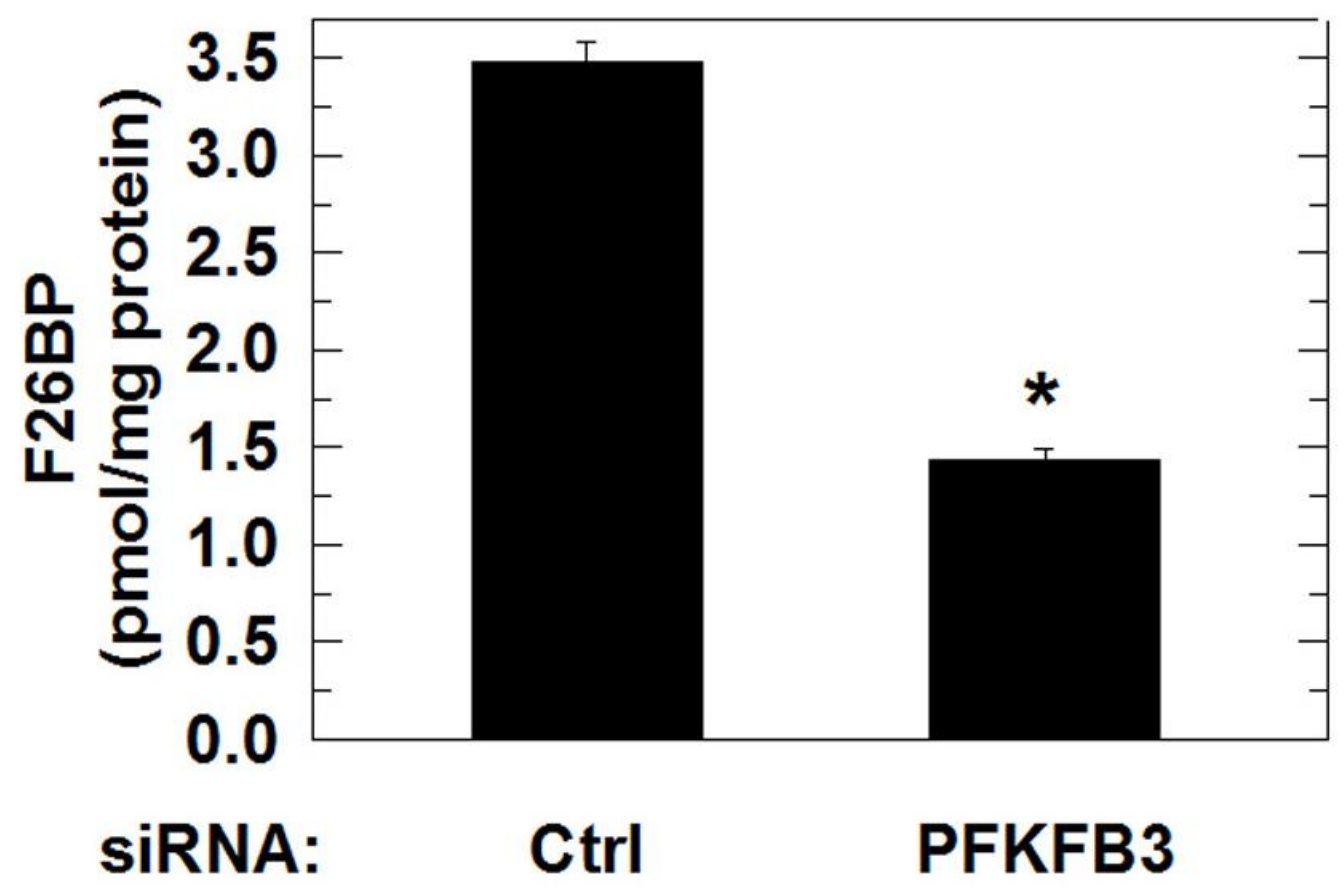


Figure 3. 2-[1- $\left.-{ }^{14} \mathrm{C}\right]$-deoxy-D-glucose uptake is reduced by PFKFB3 knockdown. 48 hours after transfection with either control (ctrl) or PFKFB3specific siRNA (PFKFB3), glucose uptake was measured using $2-\left[1-{ }^{14} \mathrm{C}\right]-$ deoxyD-glucose. Data are presented as the mean counts \pm SD from three experiments. $(p$ value $<0.05)$ 
Figure 3.

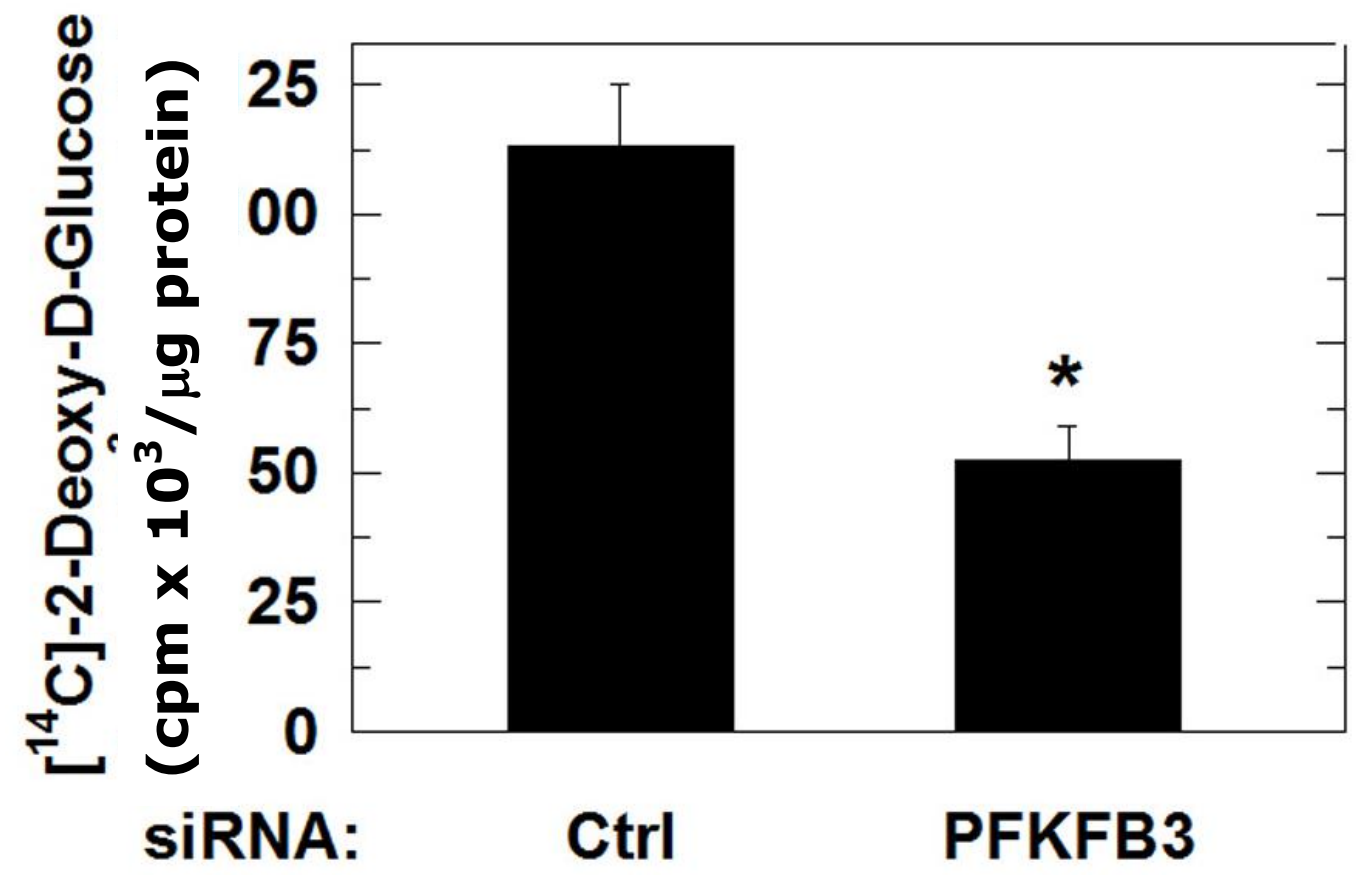




\section{PFKFB3 knockdown results in activation of autophagy}

Glucose starvation is known to increase autophagy in a number of model systems. Thus we sought to determine if the decrease in glucose uptake due to knockdown of PFKFB3 similarly increased autophagy in our model system. Four measures of autophagy were used including protein markers LC3-II and p62, acridine orange immunofluorescence, and electron microscopy. Knockdown of PFKFB3 resulted in an 8-fold increase in LC3-II protein levels relative to the negative control ( $p$ value $<0.05$ ). Addition of bafilomycin $\mathrm{A} 1$, a vacuolar type $\mathrm{H}_{+-}$ ATPase that inhibits lysosomal function, was used to block LC3-II degradation

and resulted in a further increase in LC3-II, indicating autophagic flux is increased rather than merely a block in LC3-II degradation (Figure 4) [108, 109]. As an additional measure of autophagy, protein levels of p62, a ubiquitin-binding scaffold protein that plays a role in targeting of cargo to autophagosomes, where it is also degraded, was measured. Knockdown of PFKFB3 resulted in a $~ 90 \%$ decrease ( $p$ value $<0.05)$ in p62 protein levels (Figure 4) [110]. 
Figure 4. Protein markers of autophagy, LC3-II and p62, are altered after knockdown of PFKFB3. 48 hours after transfection with either control (ctrl) or a siRNA directed against PFKFB3 (PFKFB3), LC3-II protein levels were determined using Western blotting. Treatment with 1nM bafilomycin A1 (Baf A1) was used to determine if LC3-II levels were a result of increased autophagic flux or impaired degradation. Quantitative densitometry was performed to assess relative protein levels. LC3-II and p62 levels are expressed as the mean fold change \pm SD from three experiments relative to $\beta$ actin and control. ( $p$ value $<$ 0.05) 
Figure 4.

A.
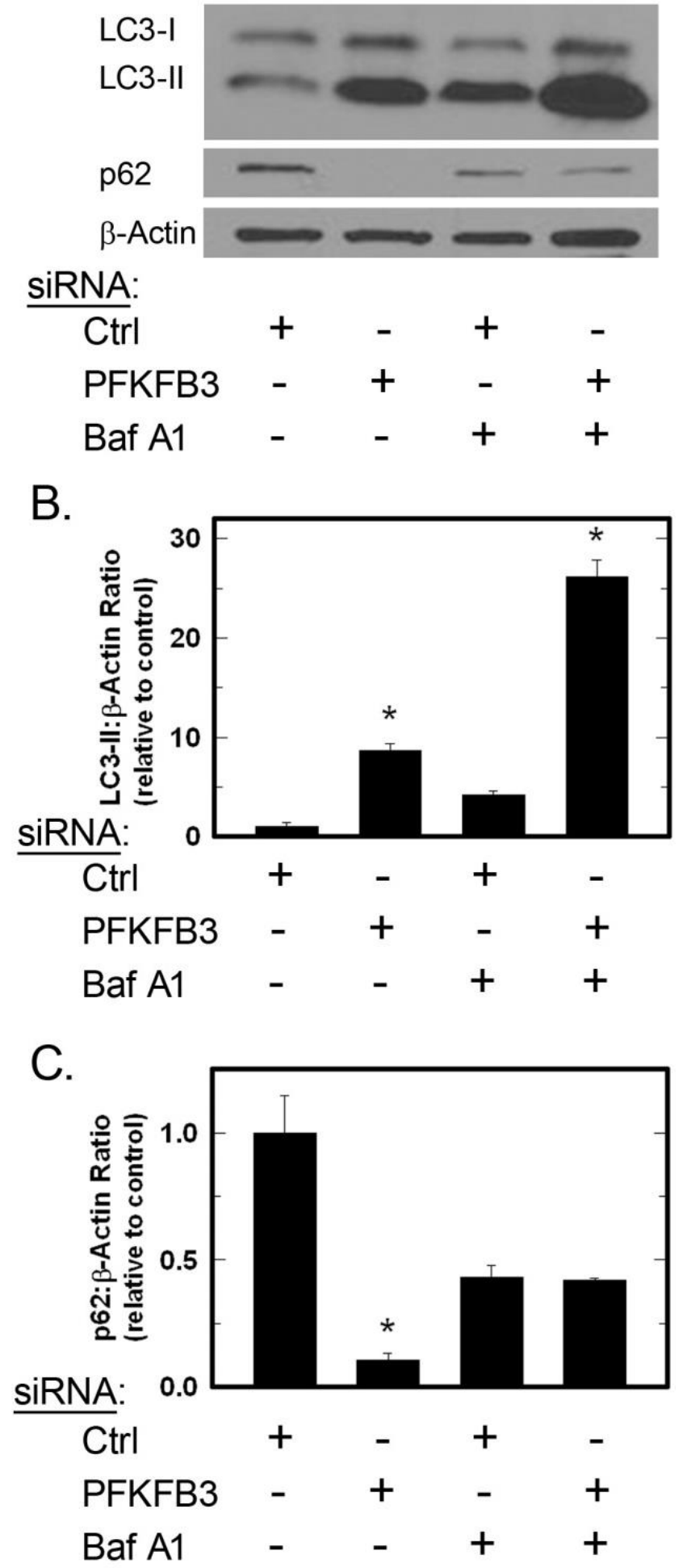
As a second measure of autophagy, acridine orange immunofluorescence was used. Acridine orange, a cell-permeable fluorescent dye, becomes protonated and trapped in acidic compartments such as lysosomes and, upon excitation (488 $\mathrm{nM})$, emits a red light $(650 \mathrm{nM})$. Acridine orange immunofluorescence was assessed by both fluorescent microscopy and by flow cytometry. HCT-116 cells transfected with PFKFB3 siRNA had a significantly higher emission of red light (650) when viewed by fluorescent microscopy (Figure $5 A)$. Acridine orange fluorescence was quantified by flow cytometry. PFKFB3 knockdown resulted in a shift in FL-3 (red) fluorescence, indicating that the PFKFB3-siRNA transfected cells had a larger quantity of acidic compartments ( $p$ value $<0.05$ ), characteristic of cells with increased autophagic activity (Figure $5 B)$.

Another methodology commonly used to study autophagy is electron microscopy. HCT-116 cells were transfected with PFKFB3 siRNA or a negative control siRNA and 48 hours post-transfection, were collected and prepared for electron microscopy. Sections were studied using a transmission electron microscope (Phillips CM12). An increased number of intracellular structures including double-membrane bound vesicles, consistent with autophagosomes were visualized in cells transfected with PFKFB3 siRNA [111]. Representative images of these cells and structures were collected (Figure 6). 
Figure 5. Acridine orange immunofluorescence is increased by PFKFB3 knockdown. After 48 hours of transfection with either control (ctrl) or PFKFB3specific siRNA, cells were stained with acridine orange and observed by fluorescent microscopy. Representative images were taken. Cells were then collected by flow cytometry to measure the relative content of acidic compartments. Data are presented as the mean percentage of cells with high acridine orange staining \pm SD. $(p$ value $<0.05)$ 
Figure 5.

A.

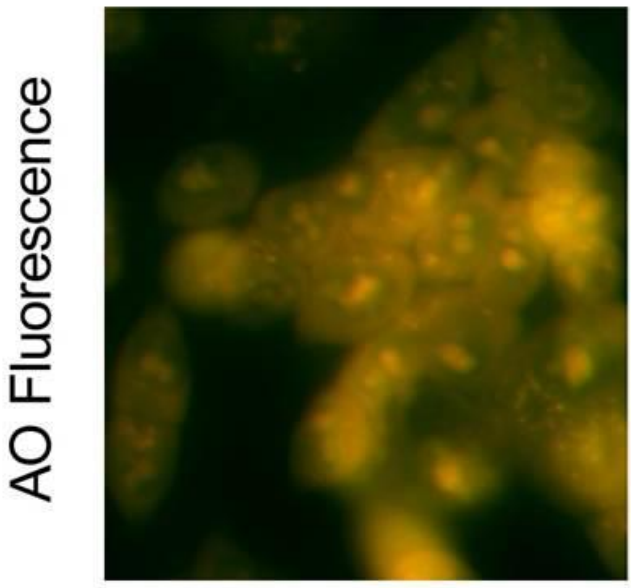

SiRNA:

Ctrl
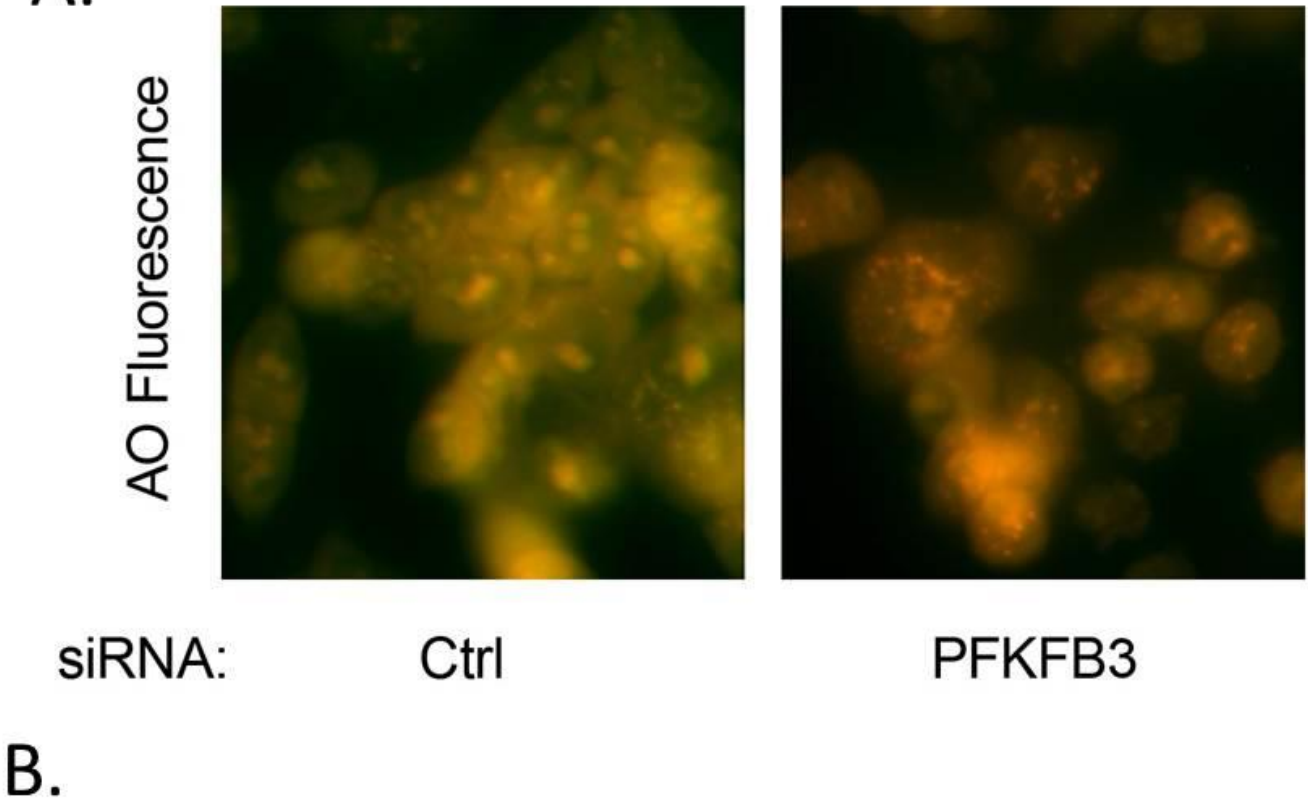

PFKFB3

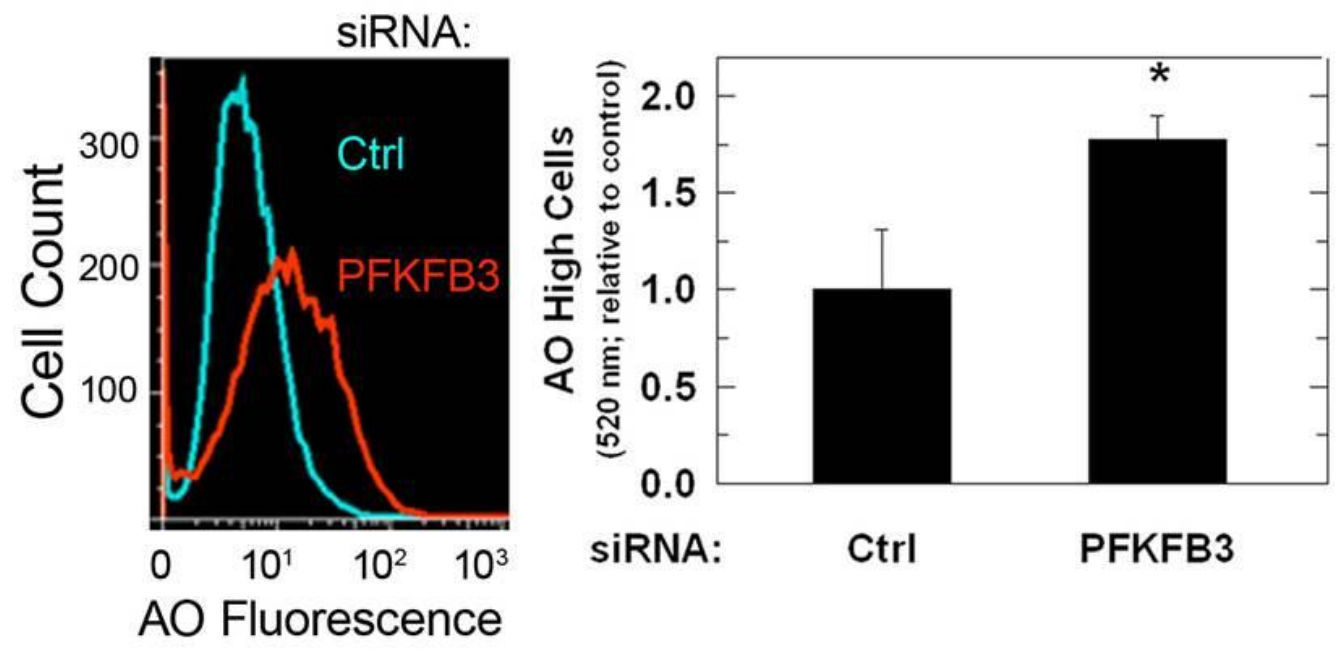


Figure 6. Autophagic structures are visible by electron microscopy after knockdown of PFKFB3. 48 hours after transfection, HCT-116 were harvested and fixed for electron microscopy. Microscopic examination of the cells treated with PFKFB3 siRNA contained intracellular structures consistent with autophagosomes (arrow). 
Figure 6.
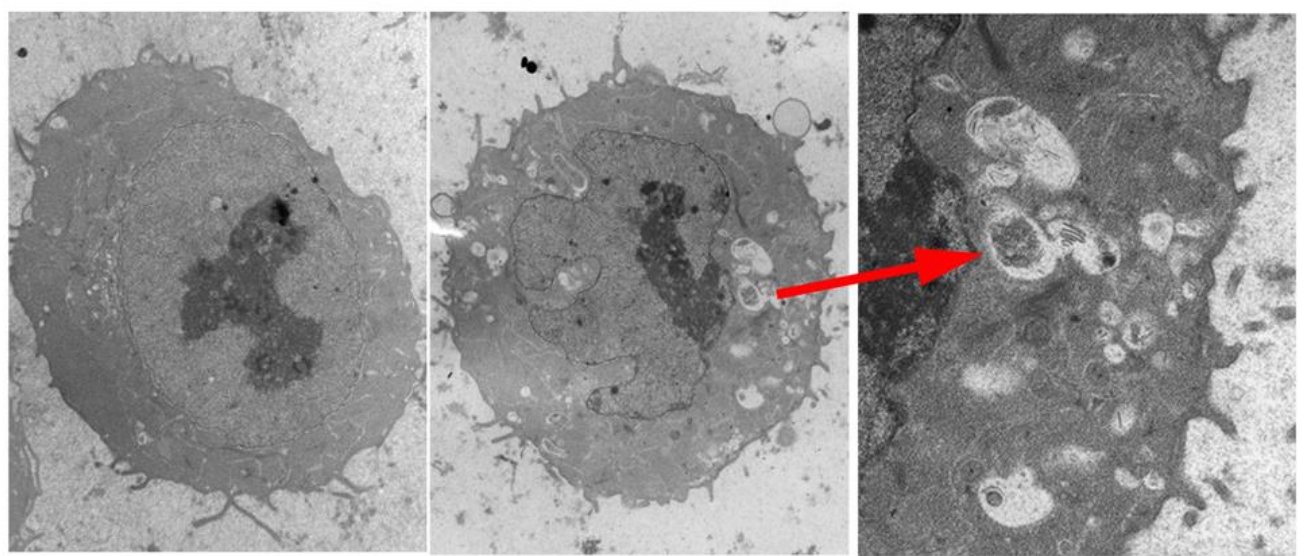

siRNA: $\quad$ Ctrl (4900X)

PFKFB3 (4900X) PFKFB3 (14000X) 


\section{Autophagy due to PFKFB3 knockdown may result from mTOR inhibition}

Protein collected from HCT-116 cells transfected with either a negative control siRNA or a PFKFB3-specific siRNA for 48 hours was used for immunoblotting to measure levels of proteins involved in the mTOR pathway. Relative to total AMPK, pAMPK was increased after 48 hours of transfection with PFKFB3 siRNA. AMPK activation is known to suppress mTOR activity which can be measured using downstream mTOR effectors p70 S6 kinase (p70S6K) and ribosomal protein S6 (S6). The phosphorylated forms of both p70S6K and S6 were decreased by PFKFB3 knockdown, relative to their total expression level (Figure 7) indicating suppression of the mTOR pathway. mTOR inhibition resulting from AMPK activation can occur when the intracellular ATP is reduced relative to AMP [112]. ATP levels after PFKFB3 knockdown were determined using a bioluminescence assay. 48 hours post-transfection, PFKFB3 knockdown resulted in a $\sim 30 \%$ decrease in ATP ( $p$ value $<0.05$ ) (Figure 8 ). Taken together, these data indicate that suppression of glucose uptake may result in decreased ATP, increased AMPK activation, mTOR pathway inhibition and resultant activation of autophagy. 
Figure 7. Protein markers downstream of mTOR are decreased by PFKFB3 knockdown. After 48 hours of transfection with either a control or a PFKFB3specific siRNA, protein expression of both phosphorylated and total levels of downstream mTOR targets, p70S6K and ribosomal protein S6 (S6) were measured using Western blotting. Activating phosphorylation of a well-studied upstream inhibitor of mTOR activity, AMPK, was also measured. Quantitative densitometry is reported as phosphorylated relative to total protein for AMPK, p70S6K and S6 and relative to $\beta$ actin for LC3. All are relative to control. Data are presented as the mean fold change \pm SD of three experiments. ( $p$ value $<$ 0.05) 
Figure 7.
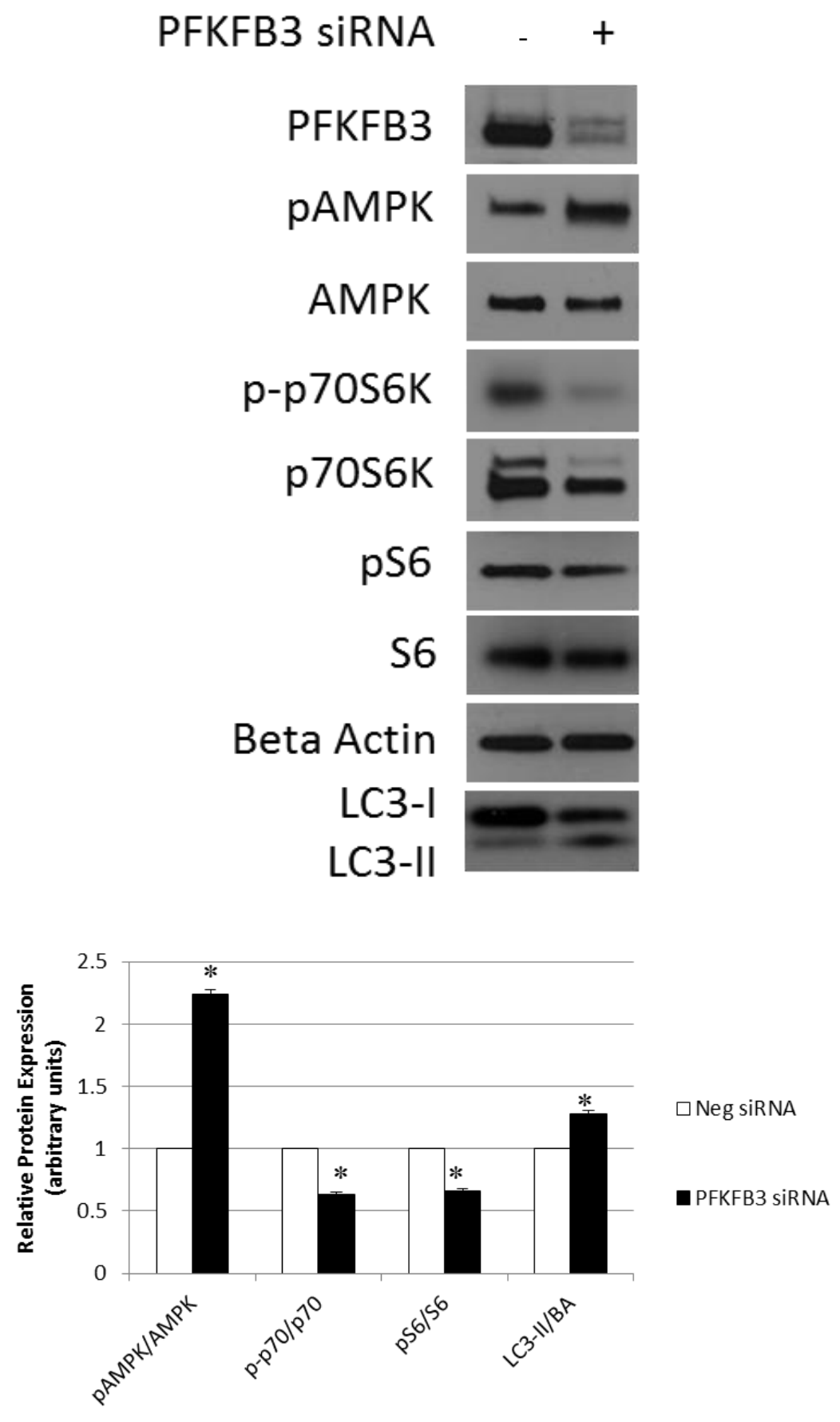
Figure 8. PFKFB3 knockdown reduces ATP levels. HCT-116 cells were transfected with either a control or a PFKFB3-specific siRNA. 48 hours after transfection cells were harvested for ATP determination. ATP was measured using a bioluminescence assay and data are presented as the mean \pm SD of ATP relative to control from three experiments. $(p$ value $<0.05)$ 
Figure 8.

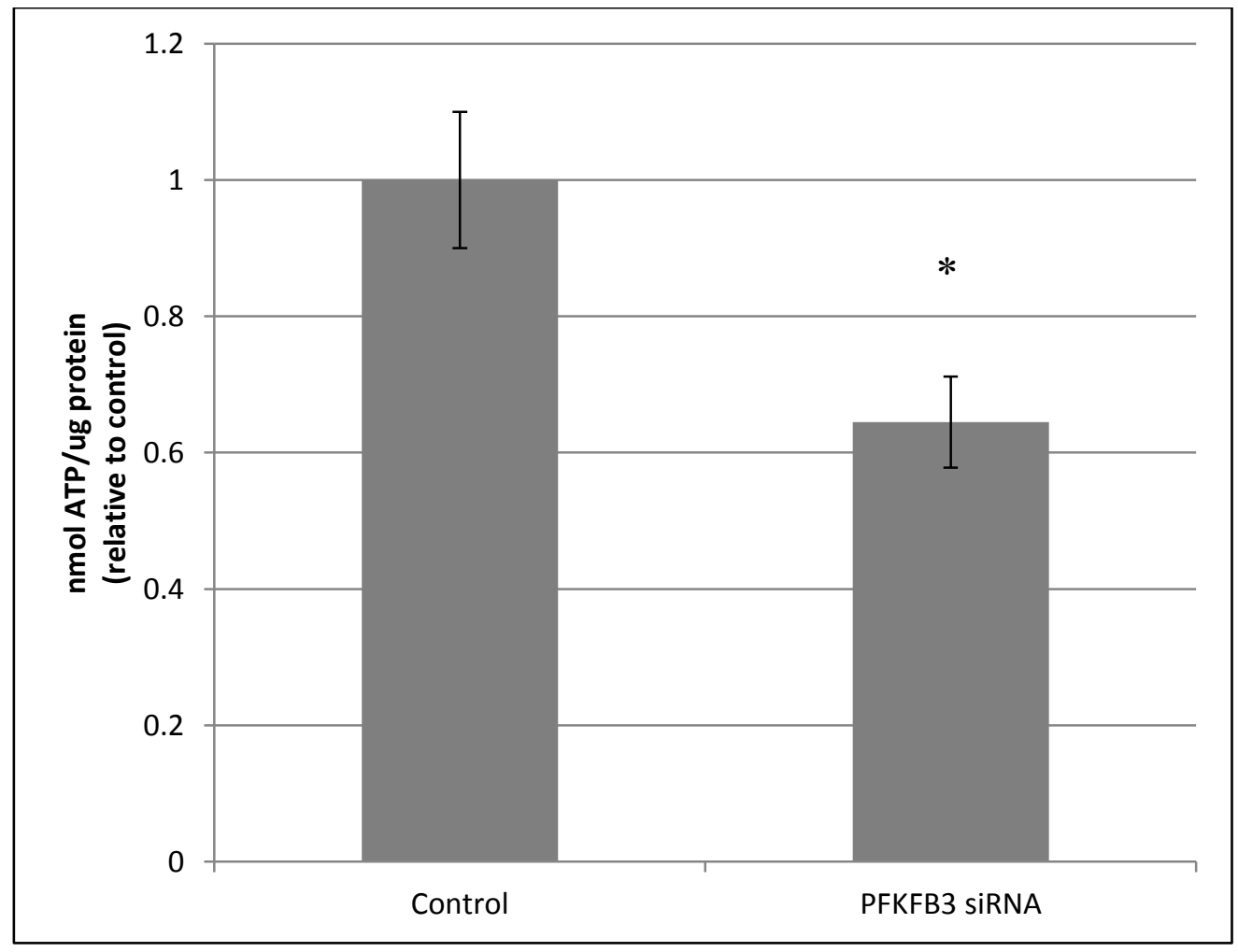




\section{Discussion}

Members of the 6-phosphofructo-2-kinase/fructose-2,6-biphosphatase (PFKFB) family have been shown to be highly expressed in neoplastic relative to normal tissue $[19,20]$. This increase in expression occurs concurrently with increased glucose uptake and utilization by tumors. For these reasons, the family of PFKFBs has become an attractive target for anti-cancer drug development. The PFKFB3 isoform is of particular interest in this pursuit due to the considerable imbalance of this enzyme's kinase to phosphatase activity. Increased activity of PFKFB3 results in the production of a large amount of F2,6BP, resulting in PFK-1 activation and increased glycolytic flux [18, 20, 26]. Inhibition of PFKFB3 has been shown to reduce glucose uptake in vitro and in vivo. Although the precise mechanism for decreased glucose uptake is not well defined, suppression of PFK-1 activity caused by decreased F2,6BP is expected to increase F6P which is in equilibrium with G6P, a potent inhibitor of hexokinase and, thus, of glucose uptake [113]. However, tumor cells are quite heterogeneous as a result of ongoing genetic alteration such that even cells within a single tumor can have distinctly different responses to a stimulus. For this reason it is important to validate each model system used when studying the 
effects of genetic manipulation of a target. To evaluate the effect of PFKFB3 knockdown, a siRNA approach was used in a transformed cell line derived from a human colon adenocarcinoma (HCT-116). Knockdown of PFKFB3 in this cell line resulted in a substantial reduction in PFKFB3 protein expression. At the same time there was also a decrease in glucose uptake and in fructose 2,6 bisphosphate production as would be expected with reduced PFKFB3 expression.

The metabolic stress caused by reduced glucose availability results in a number of cellular defense mechanisms critical to survive transitory periods of starvation. These include the suppression of energy-requiring processes by reducing the expression of biosynthetic genes, inhibiting the activity of translational machinery and halting the cell cycle [114-116]. At the same time, catabolic processes, such as autophagy, are used to recycle intracellular components in order to provide metabolic substrates which can then be used to generate energy as well as to remove potentially harmful intracellular material such as damaged mitochondria [41, 44, 117-119]. To determine if the reduced glucose uptake due to knockdown of PFKFB3 caused cells to activate this catabolic process, autophagy markers were assessed.

Knockdown of PFKFB3 in this model system resulted in changes in protein markers involved in the autophagic process. There was an increase in the lipidated form of the autophagosomal membrane protein LC3 and a decrease in protein levels of cargo protein p62 in cells transfected with PFKFB3 siRNA. Microtubule-associate protein 1 light chain 3-II (LC3-II) is a component of the 
autophagosomal membrane. LC3 is cleaved to LC3-I which liberates a Cterminal glycine that allows the conjugation to phosphatidylethanolamine (PE) whereupon it can target the autophagosomal membrane. Although counterintuitive, the heavier LC3-II migrates faster than LC3-I due to its hydrophobicity, and is seen as the lower band in Western blotting (Figure 4). [88, 120]. Increased LC3-II can indicate either increased autophagic synthesis or reduced autophagic degradation. The addition of bafilomycin A1, an inhibitor of the vacuolar type $\mathrm{H}_{+}-\mathrm{ATPase}$, allows for the determination of autophagic flux by inhibiting lysosomal acidification and blocking degradation of LC3-II [88, 121, 122]. The further increase in LC3-II protein observed in the presence of bafilomycin A1 after knockdown of PFKFB3 indicates that knockdown is inducing autophagy rather than merely causing a block in LC3-II degradation. PFKFB3 knockdown also resulted in decreased p62 protein levels. p62 is an autophagy cargo receptor protein that contains an LC3-interacting region that targets it and its cargo to the autophagosome. In autophagy competent cells, this cargo protein is degraded along with autophagosomal contents resulting in decreased total levels [123].

Additionally, knockdown of PFKFB3 resulted in cells with a higher volume of acidic compartments as measured using acridine orange staining, consistent with increased autophagy. When visualized by electron microscopy, PFKFB3 knockdown also resulted in the appearance of autophagosomal structures. Taken together, these data support the induction of autophagy due to knockdown of PFKFB3. 
Based on these observations, it appears that cells respond to PFKFB3 knockdown by increasing autophagic processing. Protein expression suggests that this induction is via the energy sensing AMPK/mTOR pathway based on an increased amount of phosphorylated AMPK and a decrease in the phosphorylated forms of downstream mTOR effectors p70 S6 kinase and ribosomal protein S6. However, because gene silencing is not currently a widely used cancer therapy technique, the significance of these studies in the setting of human disease cannot be determined using knockdown methodologies alone. To increase its relevance most targeting is aimed at the identification of compounds with anti-tumor efficacy. The interest in PFKFB3 as an anti-cancer target has resulted in the identification and validation of a number of compounds that inhibit enzyme function. In order to determine if these observations made using genetic knockdown might result in meaningful advances in tumor therapy, further studies were conducted using a PFKFB3 small molecule inhibitor that had previously been shown to inhibit PFKFB3 enzyme function. 


\section{CHAPTER III}

\section{INHIBITION OF PFKFB3 USING 3-(3-PYRIDINYL)-1-(4-PYRIDINYL) 2-PROPEN-1-ONE (3PO), RESULTS IN AUTOPHAGIC INDUCTION}

\section{Introduction}

The interest in PFKFB3 as a drug target for cancer treatment has now resulted in the identification of small molecule inhibitors of enzyme activity [53, 76]. Molecular modeling allowed for the computational screening of a large number compounds with the potential to bind the fructose-6-phosphate binding pocket of PFKFB3. Further validation of selected compounds resulted in the identification of a compound with dose-dependent inhibition of PFKFB3, termed 3-(3-pyridinyl)-1-(4-pyridinyl)-2-propen-1-one (3PO) (Figure 9). This compound was shown to suppress glucose uptake and F2,6BP production and, importantly, seemed to be cancer-specific due to the selective suppression of growth of transformed cells relative to untransformed cells [53]. Using this inhibitor, we were also able to show reduced glucose uptake and F2,6BP in our model system. 
Since a reduction in PFKFB3 activity results in decreased cellular glucose uptake, and glucose starvation is an established stimulus for increased autophagic flux, it was hypothesized that starvation due to PFKFB3 inhibition would increase autophagic flux. In the following results section, treatment of transformed cells with $3 \mathrm{PO}$ is shown to result in increased autophagy. Interestingly, the mechanism by which autophagy is induced following drug treatment does not appear to be the same as that observed using siRNA mediated knockdown. Although PFKFB3 inhibition using 3PO results in decreased ATP, this change does not occur until long after the effects on metabolism. The very early effects of $3 \mathrm{PO}$ on glucose uptake and F2,6BP relative to that with siRNA-mediated knockdown pointed to another source for autophagic induction, oxidative stress. 
Figure 9. 3-(3-pyridinyl)-1-(4-pyridinyl)-2-propen-1-one (3PO), was designed to inhibit PFKFB3 activity. Computational modeling led to the identification of a small molecule inhibitor of PFKFB3 termed -(3-pyridinyl)-1-(4-pyridinyl)-2-propen1-one (3PO) (Figure 9A). This compound can be seen within the PFKFB3 binding pocket (Figure 9B) [53]. 
Figure 9.

A.

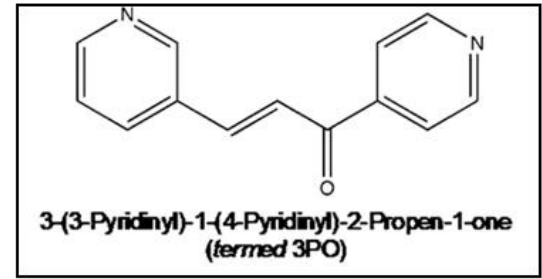

B.
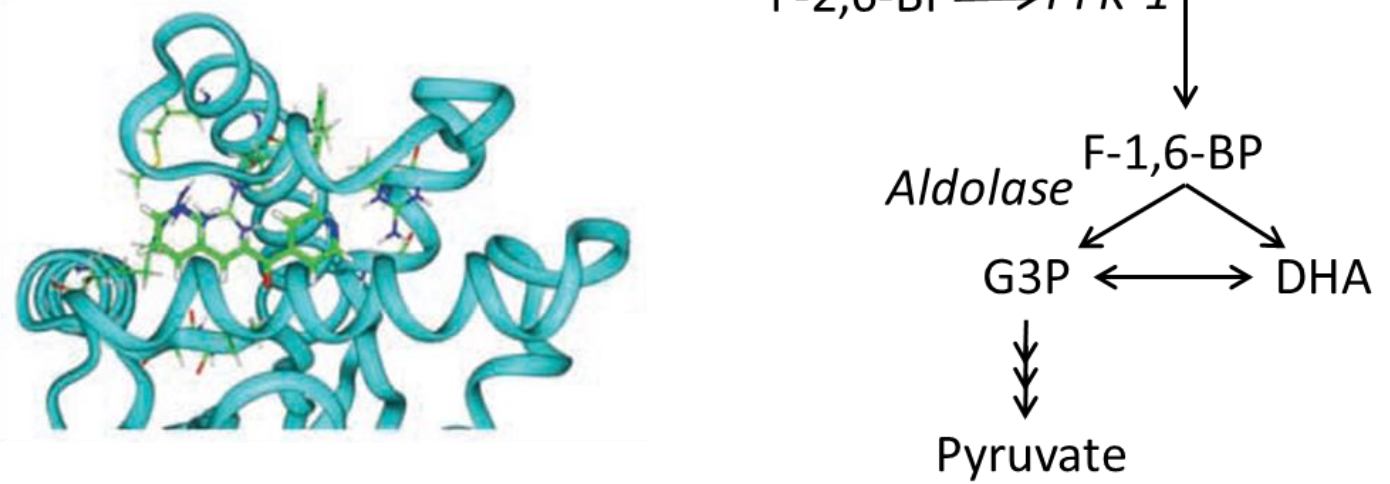


\section{Materials and Methods}

\section{Cell Culture}

Cells were cultured as described in Chapter II.

\section{Drug Treatment}

3-(3-pyridinyl)-1-(4-pyridinyl)-2-propen-1-one (3PO) (Advanced Cancer Therapeutics). Samples in which bafilomycin A1 was used were treated with 1nM bafilomycin A1 (Sigma, St. Louis, MO) 24 hours prior to harvest.

\section{Protein Extraction and Immunoblotting}

Protein extraction and Western blotting were performed as described in Chapter II.

\section{$\underline{\text { F2,6BP Assay }}$}

Intracellular fructose 2,6 bisphosphate (F2,6BP) levels were measured after two or eight hours of drug treatment as described in Chapter II. 


\section{Glucose Uptake Assay}

Glucose uptake after two or eight hours of treatment was measured using $2-\left[1-{ }^{14} \mathrm{C}\right]$-deoxy-D-glucose as described in Chapter II.

\section{Acridine Orange Immunofluorescence}

Acridine orange staining and detection was determined as described in Chapter II.

\section{Electron Microscopy}

HCT-116 cells were prepared for electron microscopy 24 hours after drug treatment as described in Chapter II.

\section{$\underline{\text { ATP Determination }}$}

ATP levels were determined after 6,12 or 24 hours of treatment as described in Chapter II.

\section{Glutathione Measurement}

GSH-Glo Glutathione Assay (Promega) was used to measure total glutathione levels following manufacturer's protocol. Briefly, cells were lifted and washed in PBS. 10,000 cells from each sample were added each well of an opaque 96-well plate. Cells were lysed with passive lysis buffer containing luciferin-NT $( \pm$ NEM 
for the measurement of GSSG). Luciferin generation reagent containing DTT, glutathione-S-transferase and glutathione reaction buffer was added and the plate was incubated at room temperature for 30 minutes. Luciferin detection reagent was added and plate was read using a luminescence reader after 15 minute incubation at room temperature. Glutathione was calculated based on a standard curve generated using 0.25 to $16 \mu \mathrm{M} \mathrm{GSH}$.

\section{Reactive Oxygen Species Flow Cytometry}

1nM 2',7' -dichlorofluorescein diacetate (DCFDA) (Molecular Probes) was diluted in 1X PBS containing magnesium and calcium (Gibco) and added to washed cells and incubated at $37^{\circ} \mathrm{C}$ for 30 minutes before being analyzed by a flow cytometry (BD FACSCalibur, San Jose, CA). Data was analyzed using FlowJo software (TREE STAR Inc, San Carlos, CA). Results were calculated as average of triplicate samples \pm SD.

\section{Flow Cytometry for Cell Death}

Cells were stained with annexin-V labeled with FITC and propidium iodide following manufacturer's protocol (BD Biosciences). Briefly, cells were lifted and pelleted by centrifugation at 2,500 rpm for 5 minutes. Cell pellets were washed with 1X PBS and 100,000 cells were pelleted by centrifugation at 2,500 rpm for 5 minutes. Pellets were resuspended in $1 \mathrm{X}$ Binding Buffer and Annexin-V/FITC and/or Propidium lodide was added and cells were incubated in the dark at room temperature for 10 minutes. $1 \mathrm{X}$ Binding Buffer was added to increase volume 
and 10,000 events were counted for each sample using the appropriate filters for FITC and PI detection (BD FACSCalibur, San Jose, CA). Data was analyzed using FlowJo software (TREE STAR Inc, San Carlos, CA). Results were calculated as average of triplicate samples \pm SD. 


\section{Results}

\section{Small molecule inhibition of PFKFB3 decreases glucose uptake}

A small molecule designed to target the fructose-6-phosphate binding site of the PFKFB3 enzyme has previously been shown to suppress glycolysis, inhibit recombinant PFKFB3 activity, and decrease glucose uptake and fructose 2,6,bisphosphate (Figure 9). This inhibitor, 3-(3-pyridinyl)-1-(4-pyridinyl)-2propen-1-one (3PO), has also been used as an anti-tumor agent in vivo. To validate this small molecule in our model system HCT-116 cells were treated and fructose 2,6 bisphosphate levels and glucose uptake were measured. HCT-116 cells were treated with either vehicle alone or $10 \mu \mathrm{M} 3 \mathrm{PO}$. Fructose 2,6 bisphosphate was measured using an enzyme-coupled reaction and a sharp $\sim 50 \%$ drop in fructose 2,6 bisphosphate was noted only 2 hours after drug treatment (Figure 10). 3PO-treated cells were also assessed for glucose uptake using 2-[1- $\left.{ }^{14} \mathrm{C}\right]$-deoxy-D-glucose. Relative to vehicle treated cells, $\sim 35 \%$ reduced glucose uptake was observed in 3PO treated cells as early as two hours after drug treatment (Figure 11). 
Figure 10. Small molecule inhibition of PFKFB3 reduces fructose 2,6,bisphosphate (F2,6BP) levels. To determine the activity of $3 \mathrm{PO}$ in the current model system, HCT-116 cells were treated with $10 \mu \mathrm{M} 3 \mathrm{PO}$ and cells were harvested to measure fructose 2,6 bisphosphate using an enzyme coupled assay. Data are presented as the mean $\mathrm{pmol} / \mathrm{mg}$ protein $\pm \mathrm{SD}$ from three experiments. $(p$ value $<0.05)$ 
Figure 10.

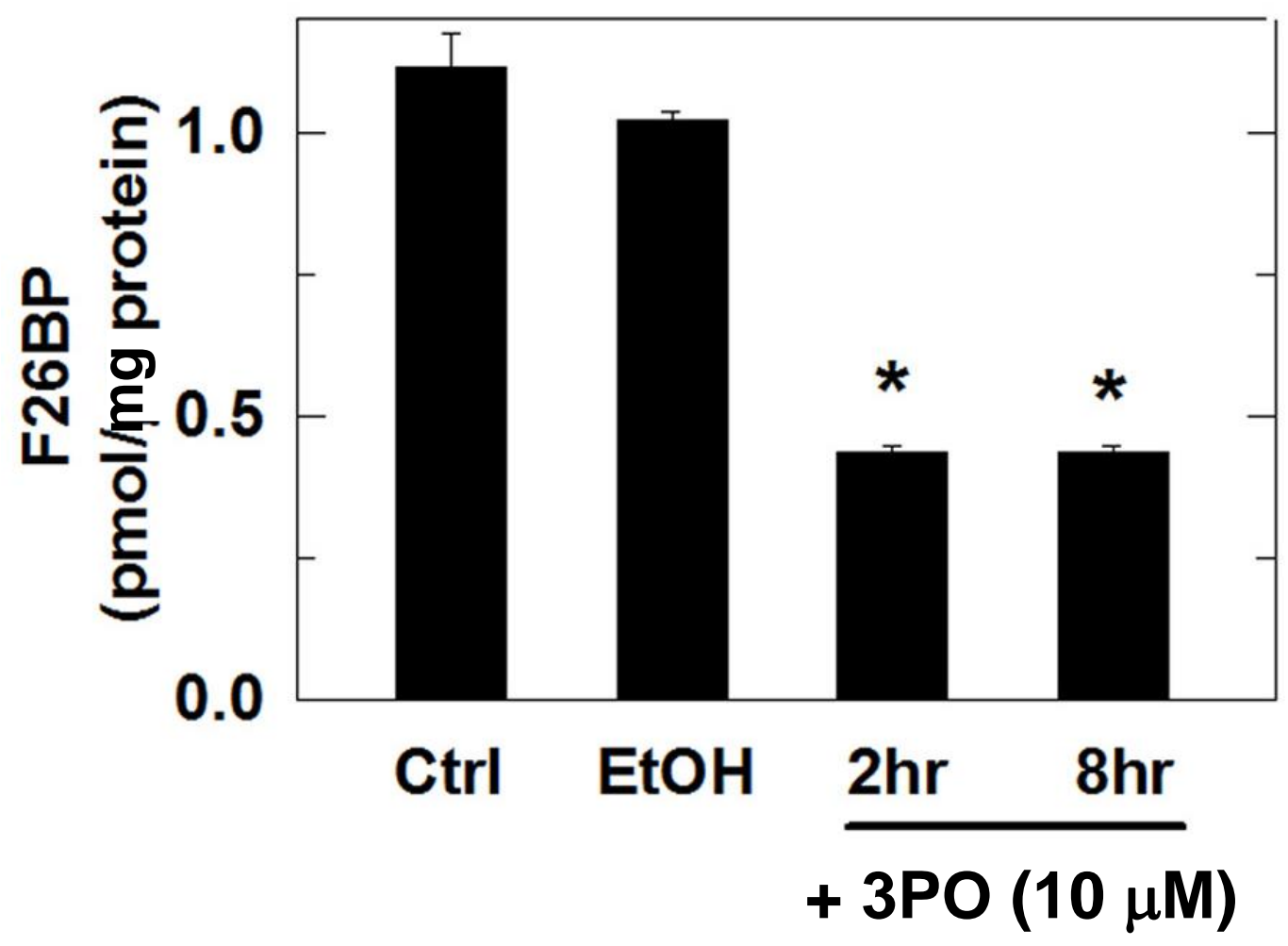


Figure 11. A small molecule inhibitor of PFKFB3, 3PO, reduces 2-[1- $\left.{ }^{14} \mathrm{C}\right]-$ deoxy-D-glucose uptake. $2-\left[1-{ }^{14} \mathrm{C}\right]-$ deoxy-D-glucose uptake was measured in HCT-116 cells treated with either vehicle or $10 \mu \mathrm{M}$ 3PO. Data are presented as the mean counts \pm SD from three experiments. $(p$ value $<0.05)$ 
Figure 11.

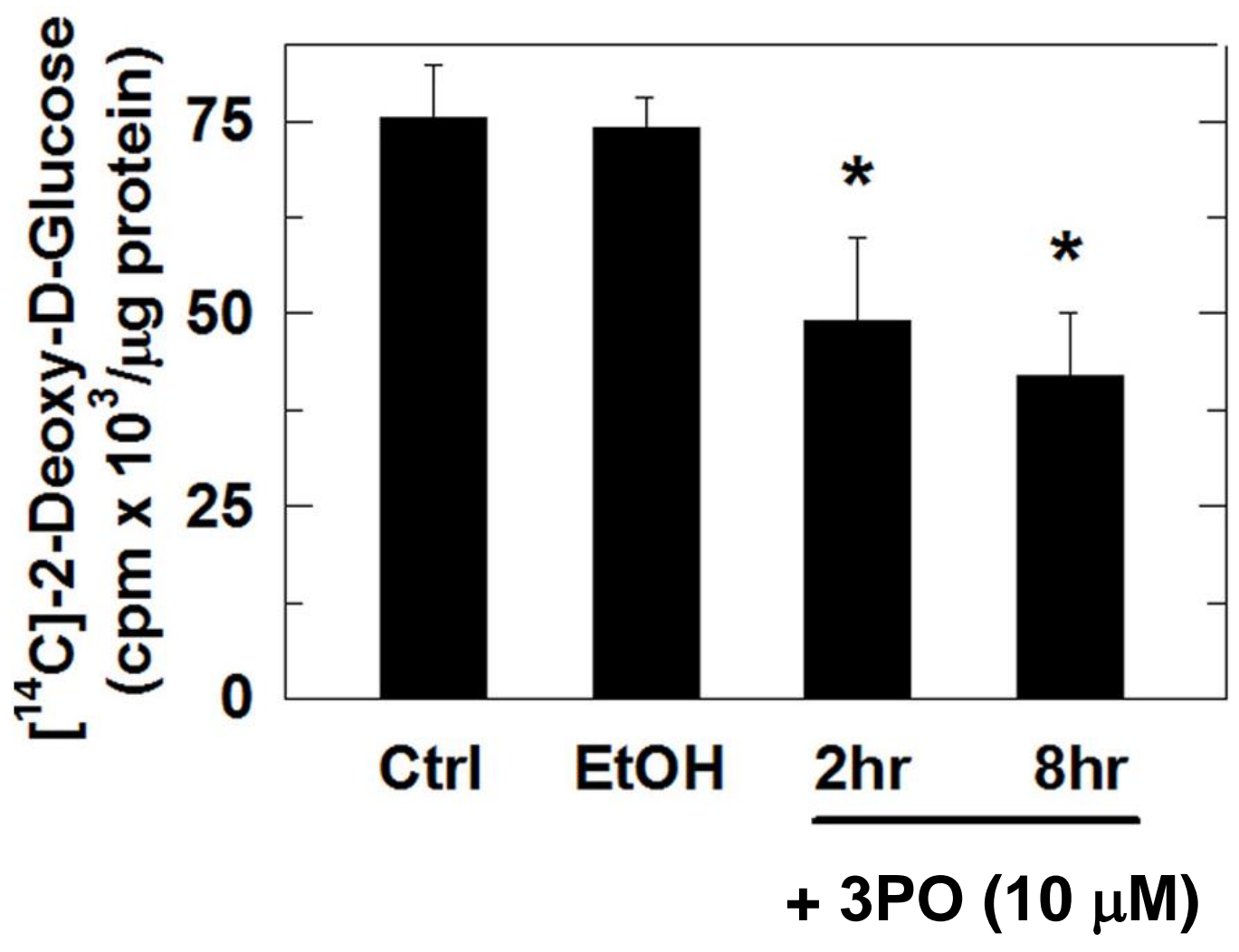




\section{Inhibition of PFKFB3 induces autophagy}

Multiple markers of autophagy were measured following small molecule inhibition of PFKFB3. Treatment of HCT-116 cells with 7.5 , 10, or $15 \mu \mathrm{M} 3 \mathrm{PO}$ for 24 hours led to the increased expression of protein marker LC3-II in a dose dependent manner. Like PFKFB3 knockdown, the LC3-II induction by 3PO is likely due to increased synthesis rather than a blockade of protein degradation as indicated by the further increase in LC3-II upon addition of bafilomycin A1 (Figure 12). 3PO also resulted in a decreased amount of p62 (Figure 12).

Treatment with $7.5,10$, or $15 \mu \mathrm{M} 3 \mathrm{PO}$ for 24 hours increased acridine orange immunofluorescence which was visualized by fluorescent microscopy (Figure 13) and quantified using flow cytometry (Figure 13). This induction of fluorescence was also noted to occur in a dose dependent manner.

HCT-116 cells treated with $10 \mu \mathrm{M} 3 \mathrm{PO}$ were examined by electron microscopy. After 24 hours of treatment, cells exposed to 3PO were noted to have numerous intracellular structures consistent with autophagosomes (Figure 14). 
Figure 12. Markers of autophagy, LC3-II and p62, are altered by 3PO. HCT116 cells were treated with either vehicle, or $7.5,10$, or $15 \mu \mathrm{M} 3 \mathrm{PO}$ for 24 hours. Addition of bafilomycin A1 (Baf A1) was used to determine if the changes in LC3II were the result of increased synthesis or impaired degradation. Quantitative densitometry was conducted and protein expression was determined relative to $\beta$ actin and to control. (LC3-II quantitation is relative to control + bafilomycin due to the absence of a visible band in the control sample). Data are presented as the mean fold change \pm SD from three experiments. $(p$ value $<0.05)$ 
Figure 12.
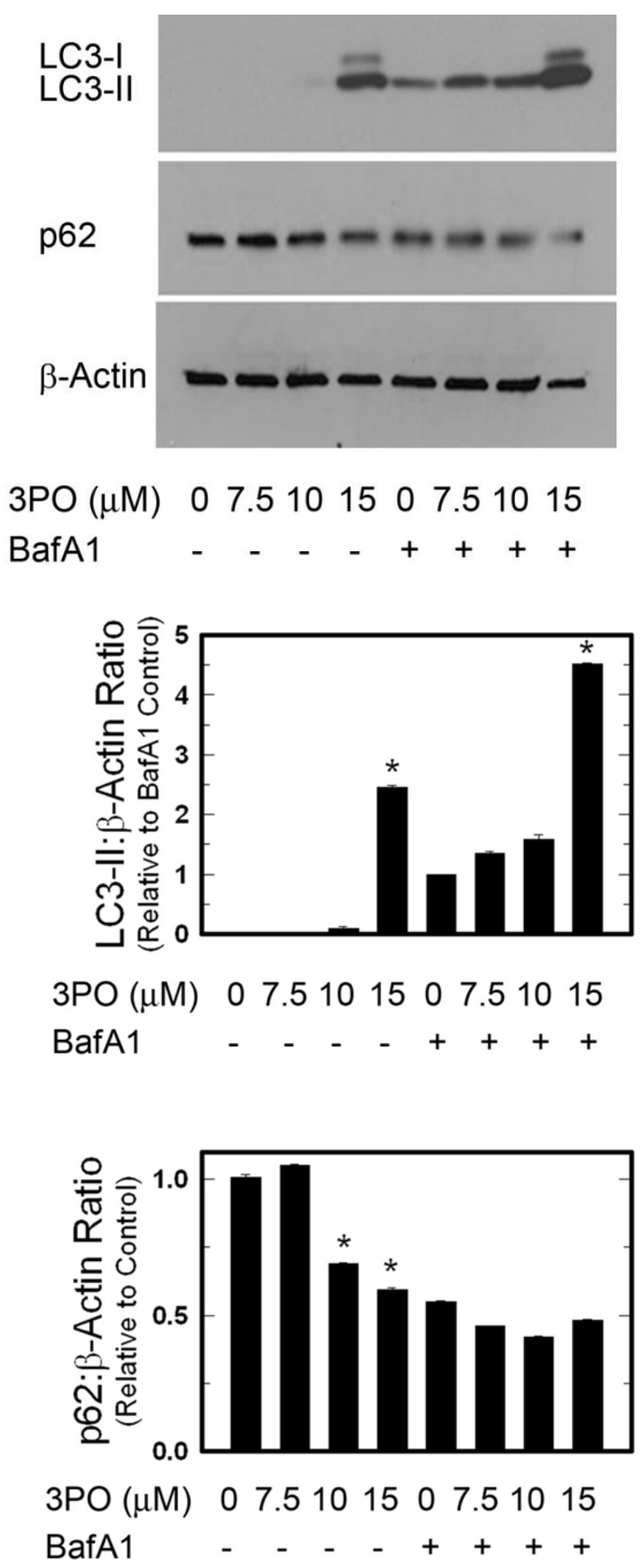
Figure 13: 3PO increases acridine orange immunofluorescence. $\mathrm{HCT}$ 116 cells were treated with either vehicle or $7.5,10$, or $15 \mu \mathrm{M} 3 \mathrm{PO}$. 24 hours after treatment, cells were stained with $1 \mathrm{ug} / \mathrm{ml}$ acridine orange for 15 minutes and then viewed using a fluorescent microscope. Representative images were captured (Figure 12A). Cells were then harvested for flow cytometry. Gating was used to quantitate the number of cells with a high $A O$ fluorescence and expressed relative to vehicle (Figure 12B, C). Data are presented as the mean relative percentages \pm SD from three experiments. $(p$ value $<0.05)$ 
Figure 13.
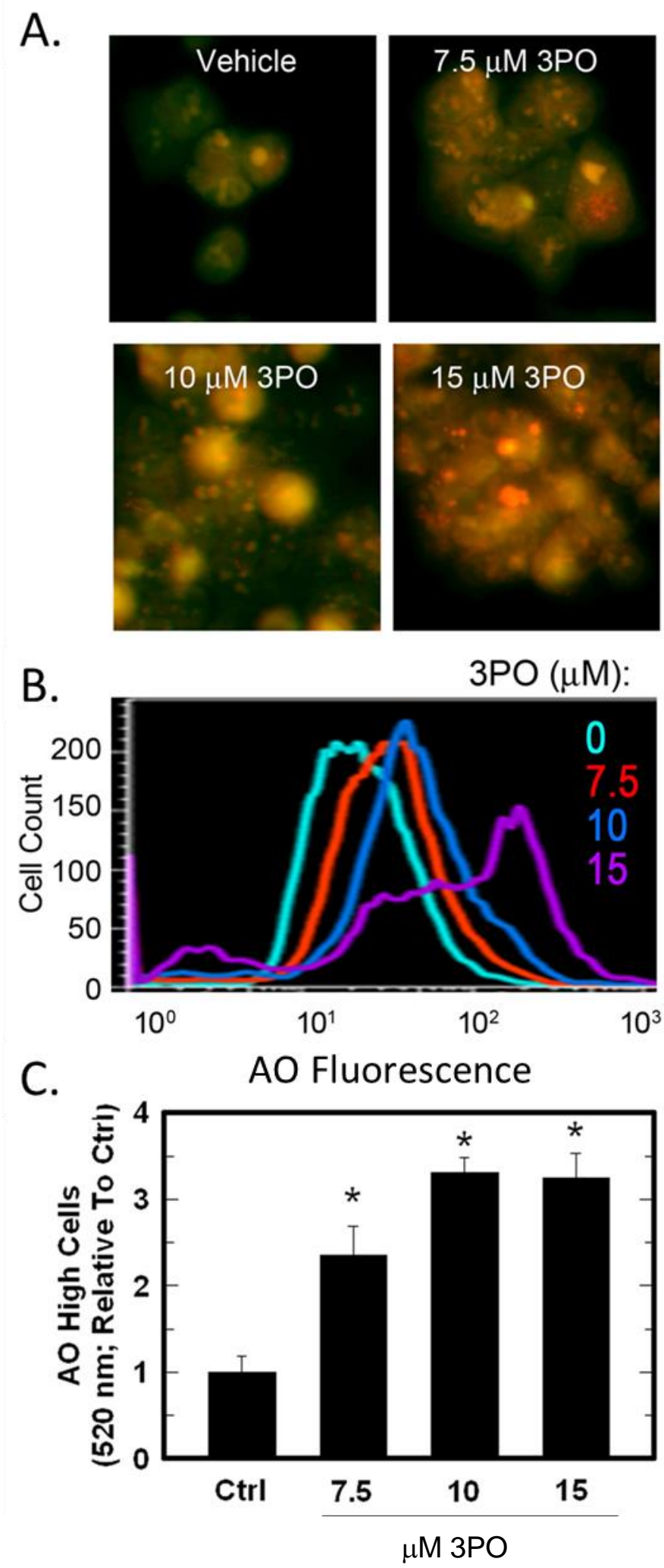
Figure 14: Inhibition of PFKFB3 results in autophagic structures visualized by electron microscopy. HCT-116 cells treated with $10 \mu \mathrm{M} 3 \mathrm{PO}$ were harvested after 24 hours and prepared for electron microscopy. Representative images were captured. Autophagic structures were seen in cells exposed to 3PO (arrow). 
Figure 14.

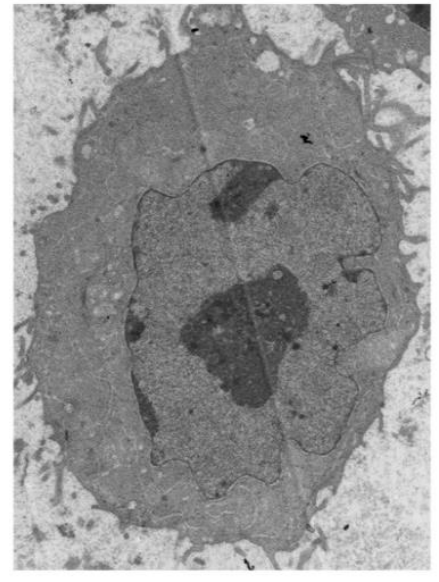

Ctrl

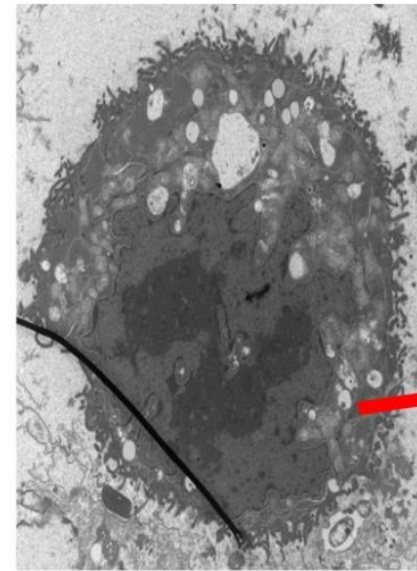

+3PO (3800X)

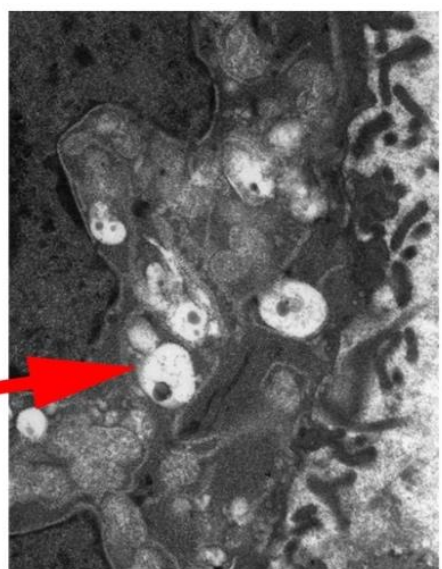

+3PO (14000X) 


\section{mTOR inhibition following 3PO treatment does not correlate with AMPK}

Protein lysates prepared from HCT-116 cells treated with $3 \mathrm{PO}(10 \mu \mathrm{M})$ were examined for protein expression and phosphorylation of downstream mTOR effectors p70S6K and S6. The levels of phosphorylated p70S6K and S6 were decreased after treatment with $3 \mathrm{PO}$, indicating mTOR inhibition similar to that seen with knockdown of PFKFB3. However, unlike the knockdown, 3PO did not result in the activation of AMPK (Figure 15). The lack of AMPK activation was appropriate, however, because ATP was not found to decrease until 24 hours after treatment (Figure 16). 
Figure 15: 3PO treatment results in decreased expression of downstream mTOR effectors. HCT-116 cells were treated with $10 \mu \mathrm{M} 3 \mathrm{PO}$ and harvested at specified times for protein lysates. Immunoblotting was performed using antibodies to detect pAMPK, AMPK, p-p70S6K, p70S6K, pS6, S6, $\beta$ actin and LC3. Quantitative densitometry was used to determine protein expression and is expressed as phosphorylated relative to total levels for AMPK, p70S6K and S6 and relative to $\beta$ actin for LC3-II. All values are expressed as fold change relative to control and are presented as the mean fold change \pm SD of three experiments. $(p$ value $<0.05)$ 
Figure 15.
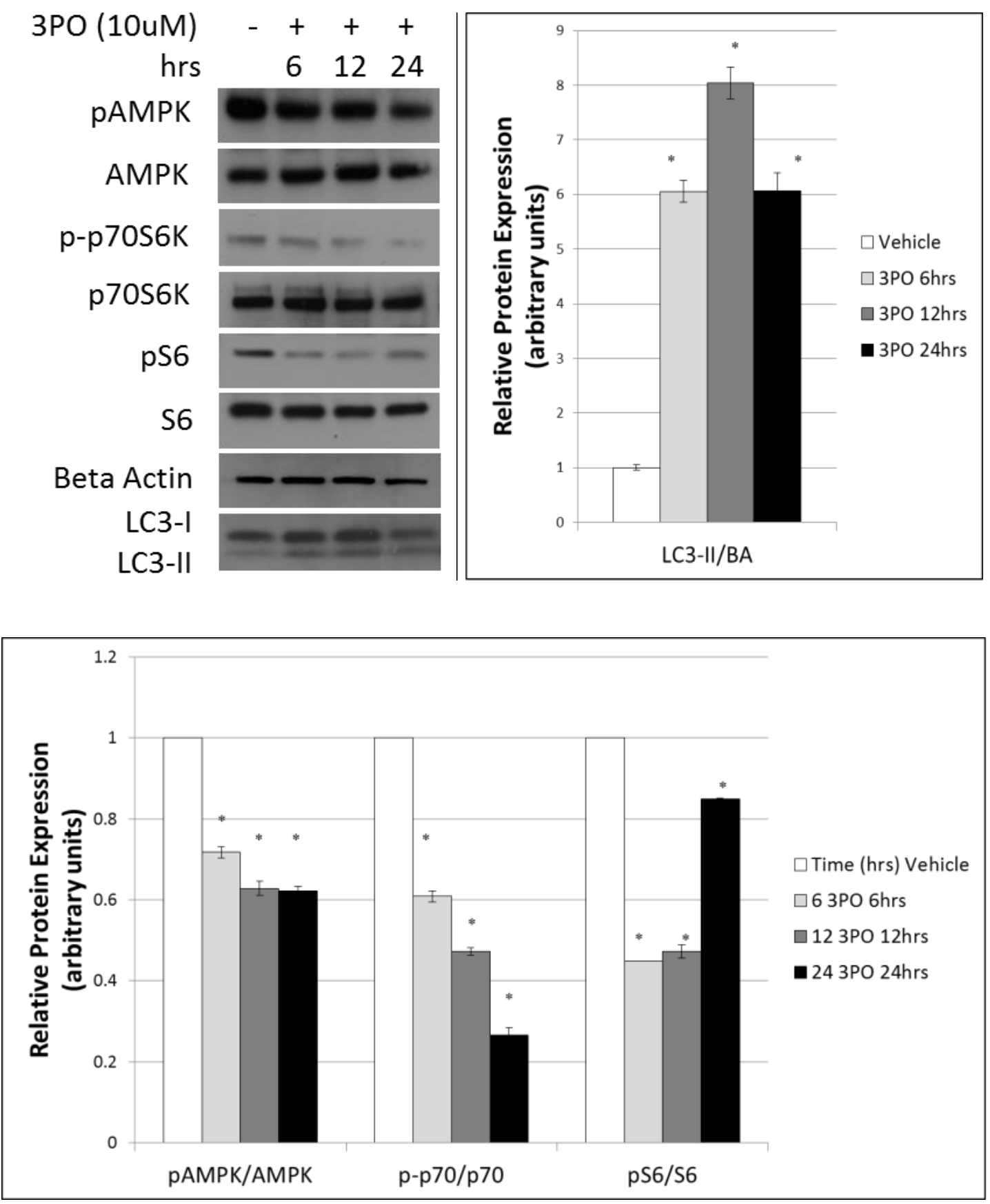
Figure 16: ATP levels are decreased by treatment with 3PO. Cells treated with $10 \mu \mathrm{M}$ 3PO were harvested at specified times and intracellular ATP was measured using a bioluminescence assay. ATP levels are expressed as mean nmol ATP/ug protein relative to control \pm SD from three experiments. ( $p$ value $<$ 0.05) 
Figure 16.

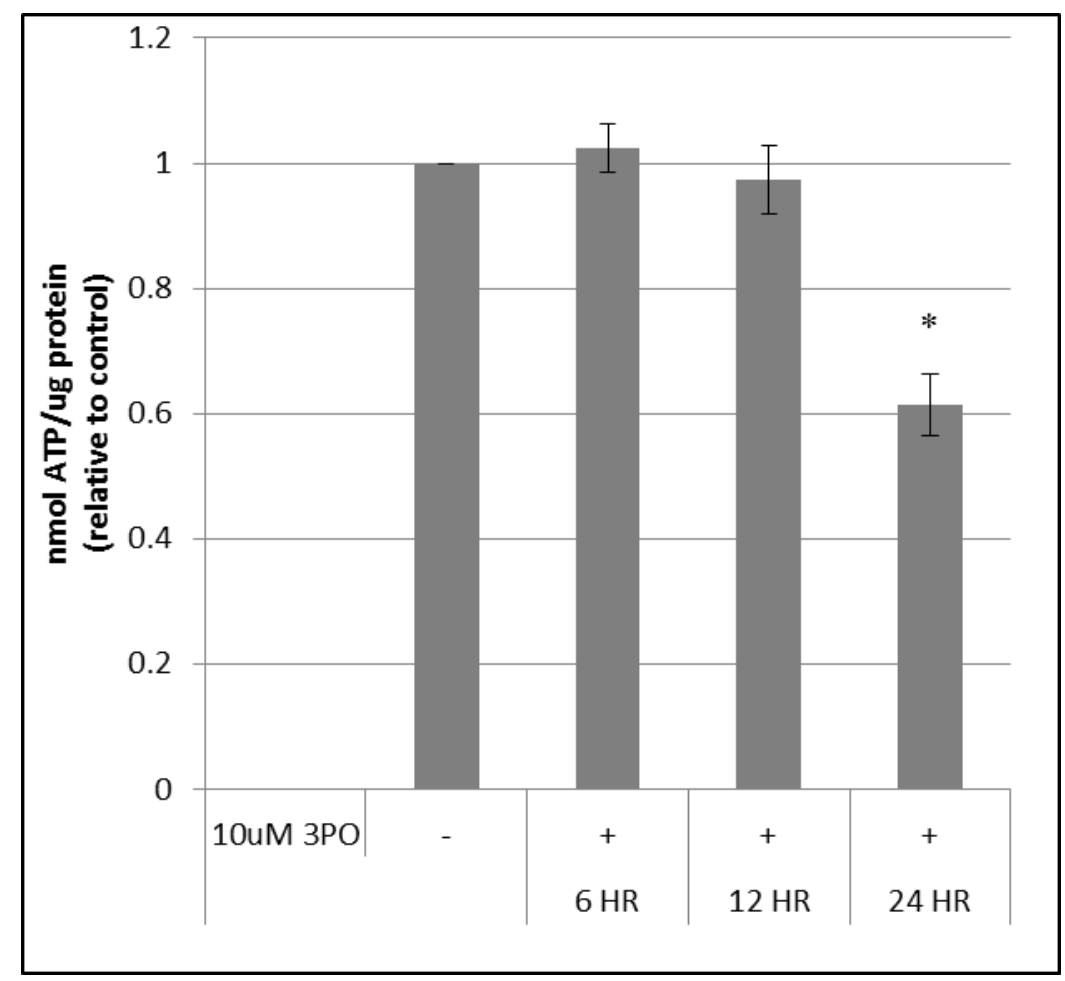




\section{PFKFB3 inhibition with 3PO increases oxidative stress}

Glucose starvation is known to induce oxidative stress [124, 125]. After determining that 3PO reduced fructose 2,6 bisphosphate and glucose uptake very soon after treatment, we sought to determine the effect of 3PO on oxidative stress, focusing on glutathione levels and reactive oxygen species. HCT-116 cells were treated with $10 \mu \mathrm{M} 3 \mathrm{PO}$ and harvested for glutathione measurement using a luminescence-based assay. After 2 hours of treatment, 3PO-treated cells had a $\sim 40 \%$ decrease in glutathione relative to control ( $p$ value $<0.05$ ) (Figure 17). To determine if this prompt change in glutathione had an impact on reactive oxygen species, a flow cytometry approach was employed. Fluorescence of 2',7' -dichlorofluorescein diacetate (DCFDA), a dye that is used to measure hydroxyl, peroxyl and other reactive oxygen species was determined by flow cytometry. Reactive oxygen species were increased by more than $50 \%$ in 3PO-treated cells ( $p$ value $<0.05$ ) (Figure 18). 
Figure 17: $3 \mathrm{PO}$ treatment causes a reduction in glutathione. Glutathione was measured using a luminescence-based assay in HCT-116 cells treated with either vehicle or $10 \mu \mathrm{M}$ 3PO. Glutathione was calculated as $\mu \mathrm{g} \mathrm{GSH} / \mu \mathrm{g}$ protein relative to vehicle treated cells and is expressed as the mean \pm SD from three experiments. ( $p$ value $<0.05)$ 
Figure 17.

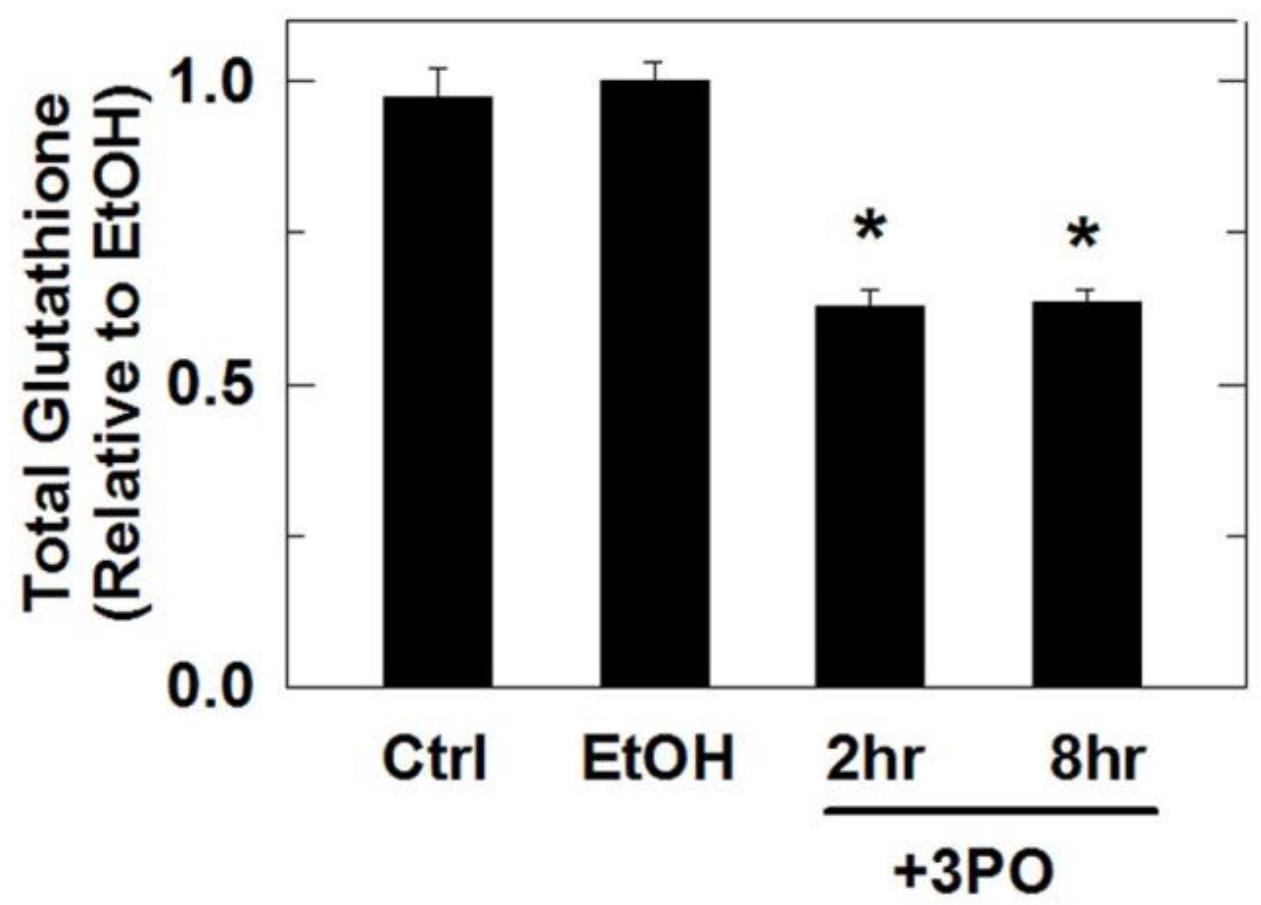


Figure 18: Reactive oxygen species are increased by treatment with 3PO. HCT-116 cells treated with either vehicle or $10 \mu \mathrm{M} 3 \mathrm{PO}$ were harvested at specified time points and loaded with 2',7'-dichlorofluorescein diacetate (DCFDA) for determination of reactive oxygen species by flow cytometry. Average geometric mean of fluorescence intensity calculated as the relative mean $\pm S D$ from three experiments is presented. ( $p$ value $<0.05$ ) 
Figure 18.

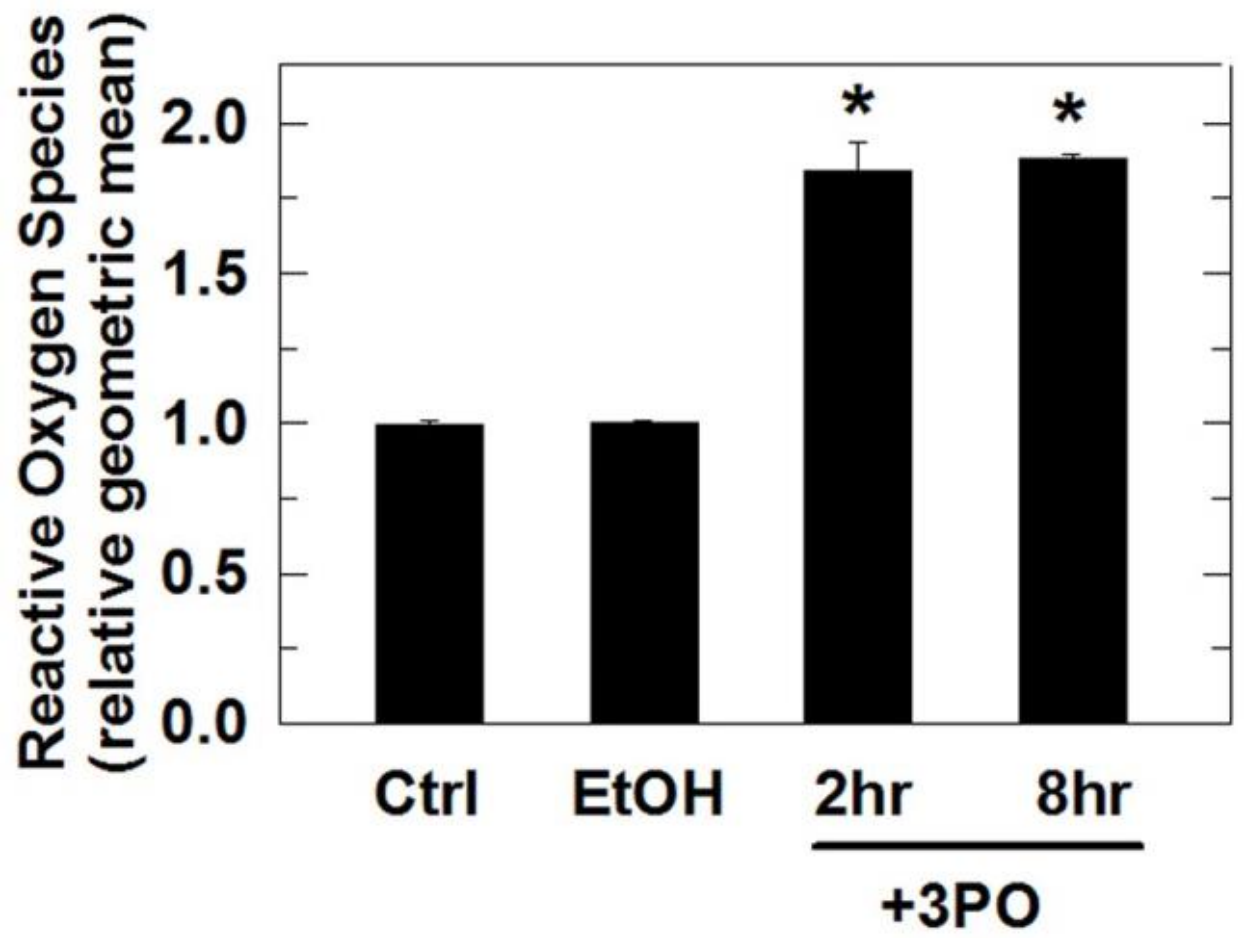




\section{Activation of autophagy due to PFKFB3 inhibition is rescued with NAC}

The correlation between oxidative stress and autophagy was further examined using the anti-oxidant $\mathrm{N}$-acetylcysteine, which can act as a precursor to the antioxidant glutathione. The drop in glutathione after $10 \mu \mathrm{M} 3 \mathrm{PO}$ treatment was partially rescued by the addition of $1 \mathrm{mM} \mathrm{N}$-acetylcysteine (Figure 19). Nacetylcysteine also partially blocked the increase in reactive oxygen species induced by 3PO, as determined by flow cytometric measurement of DCFDA fluorescence (Figure 20). This rescue of glutathione and reduction in ROS following 3PO treatment in combination with NAC also blocked the induction of autophagy as measured by loss of LC3-II (Figure 21) and a reduction in acridine orange immunofluorescence (Figure 22).

$\mathrm{N}$-acetylcysteine also rescued $\mathrm{HCT}-116$ cells from cell death due to treatment with 3PO. Flow cytometric analysis of cell death using Annexin $\mathrm{V}$ and Propidium lodide staining revealed a marked reduction in both early and late apoptotic events when $1 \mathrm{mM}$ NAC was combined with $10 \mu \mathrm{M}$ 3PO (Figure 23). 
Figure 19: N-acetylcysteine prevents 3PO induced reduction in glutathione levels. HCT-116 cells were treated with either vehicle or $10 \mu \mathrm{M} 3 \mathrm{PO} \pm 1 \mathrm{mM}$ NAC and harvested after eight hours of treatment for measurement of glutathione levels using a luminescence-based assay. Glutathione values were calculated as $\mu \mathrm{g} \mathrm{GSH} / \mu \mathrm{g}$ protein relative to control $\pm \mathrm{NAC}$ and are expressed as the mean \pm SD from three experiments. 
Figure 19.

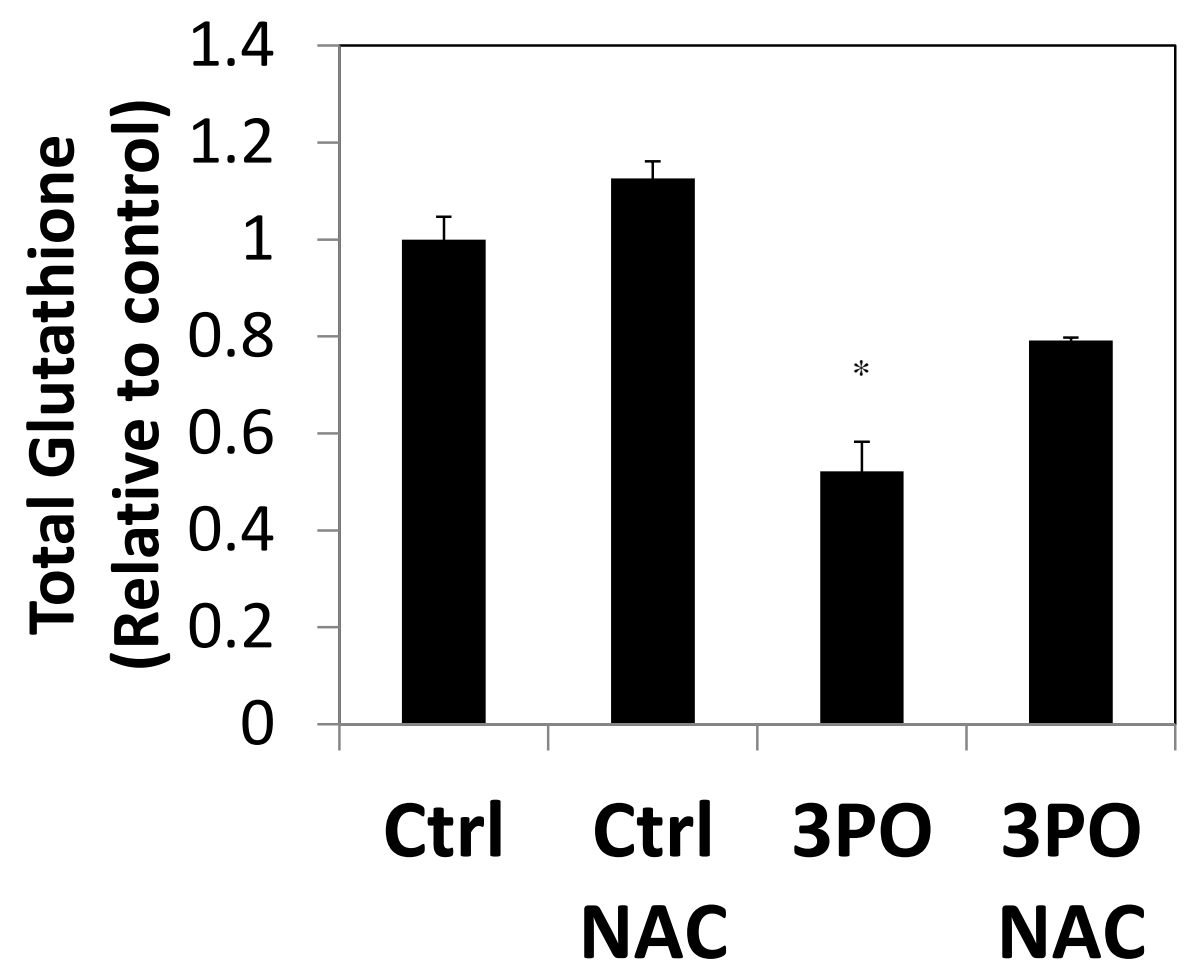


Figure 20: Reactive oxygen species production by $3 \mathrm{PO}$ is rescued by $\mathrm{N}$ acetylcysteine. 2',7'-dichlorofluorescein diacetate (DCFDA) fluorescence was measured using flow cytometry in HCT-116 cells treated for eight hours with either vehicle or $10 \mu \mathrm{M} 3 \mathrm{PO} \pm 1 \mathrm{mM}$ NAC. Average geometric mean was calculated relative to control and is expressed as the relative mean \pm SD from three experiments. $(p$ value $<0.05)$ 
Figure 20.
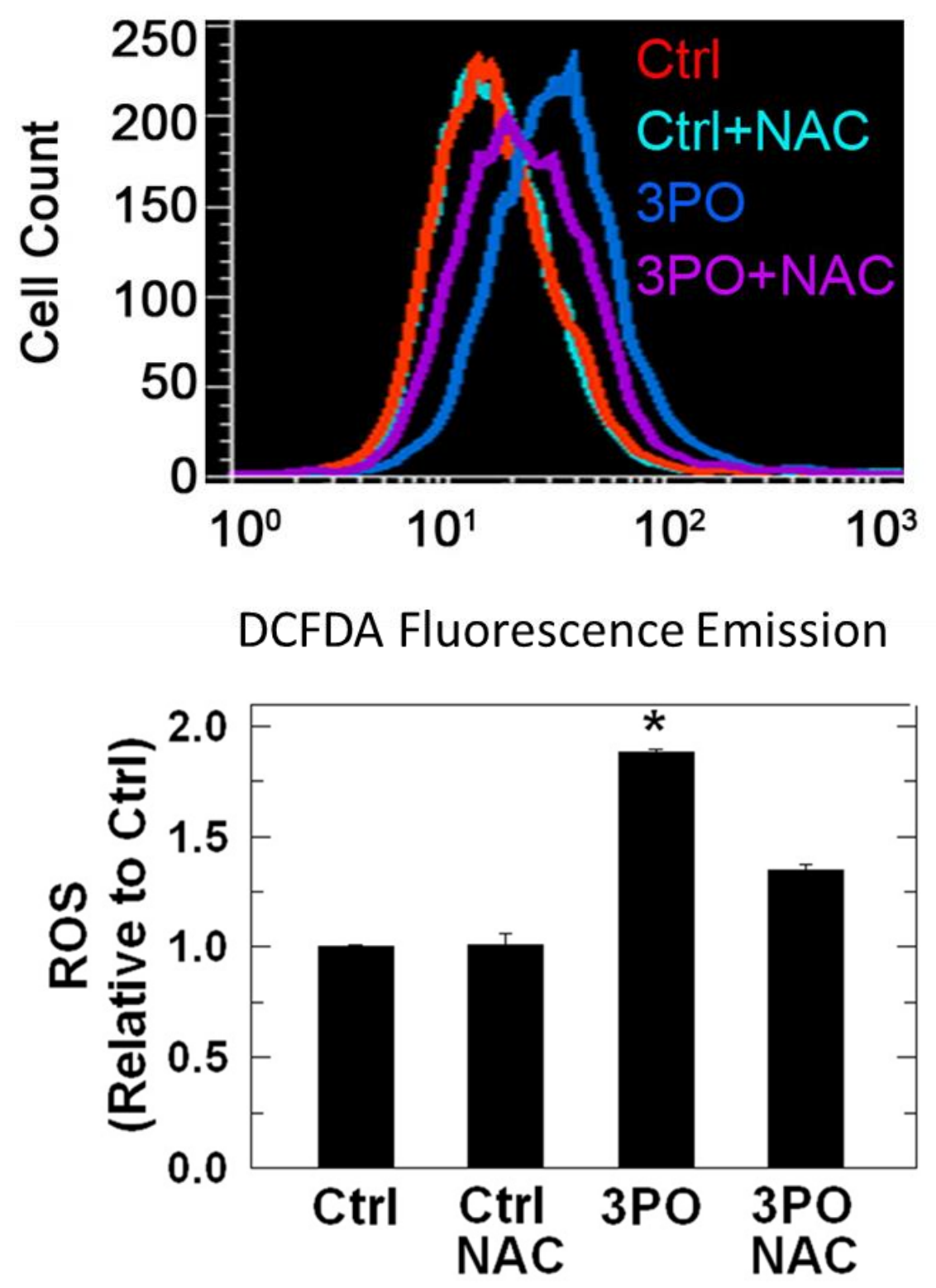
Figure 21: LC3-II induction by $3 \mathrm{PO}$ is blocked by $\mathrm{N}$-acetylcysteine. After eight hours of treatment with either vehicle or $10 \mu \mathrm{M} 3 \mathrm{PO} \pm 1 \mathrm{mM} \mathrm{NAC}$, cell lysates were prepared and LC3-II levels were determined using immunoblotting. Quantitative densitometry was performed and expressed as the fold change relative to $\beta$ actin and to control \pm SD. $(p$ value $<0.05)$ 
Figure 21.
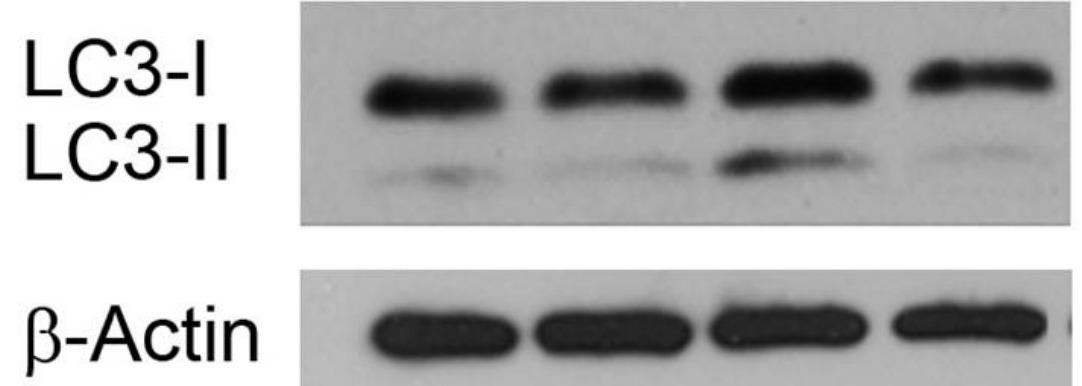

Ctrl Ctrl 3PO 3PO NAC NAC

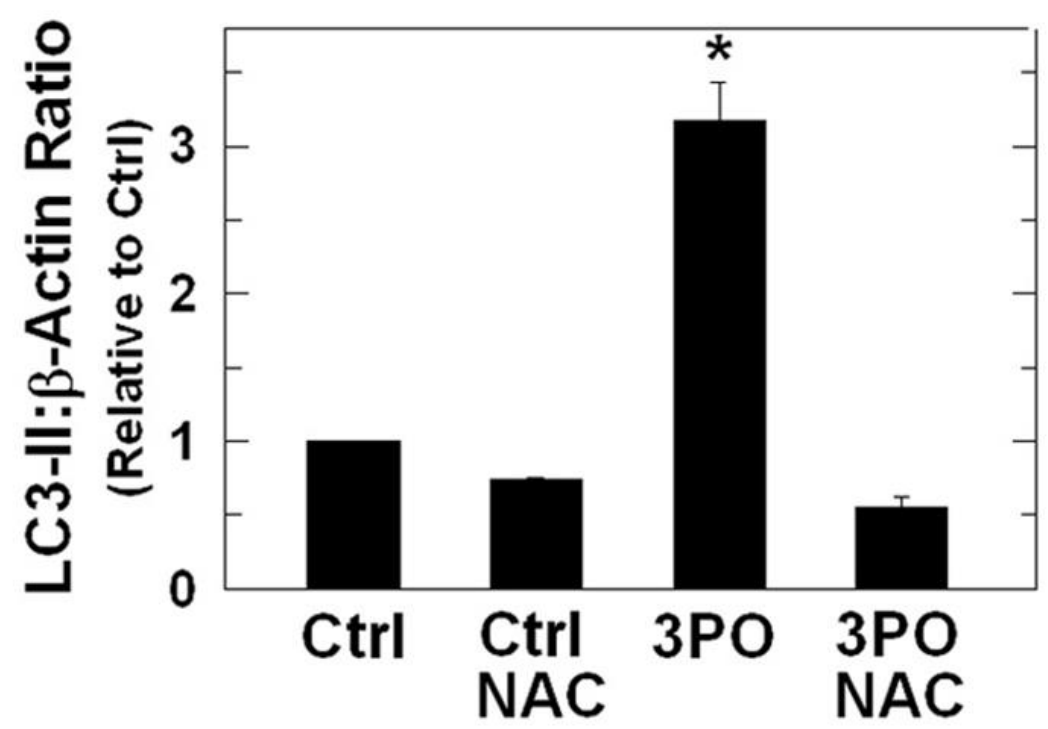


Figure 22: 3PO-induced acridine orange immunofluorescence is blocked by $\mathrm{N}$-acetylcysteine. After 24 hours of treatment with either vehicle or $10 \mu \mathrm{M} 3 \mathrm{PO}$ \pm NAC, HCT-116 cells were stained with $1 \mathrm{ug} / \mathrm{ml}$ acridine orange for 15 minutes. Fluorescence microscopy was used to capture representative images (Figure 22B). Cells were then harvested and acridine orange fluorescence was determined using flow cytometry. Fluorescence intensity was calculated as average relative to control $\pm S D($ Figure $22 A) . \quad(p$ value $<0.05)$ 
Figure 22.
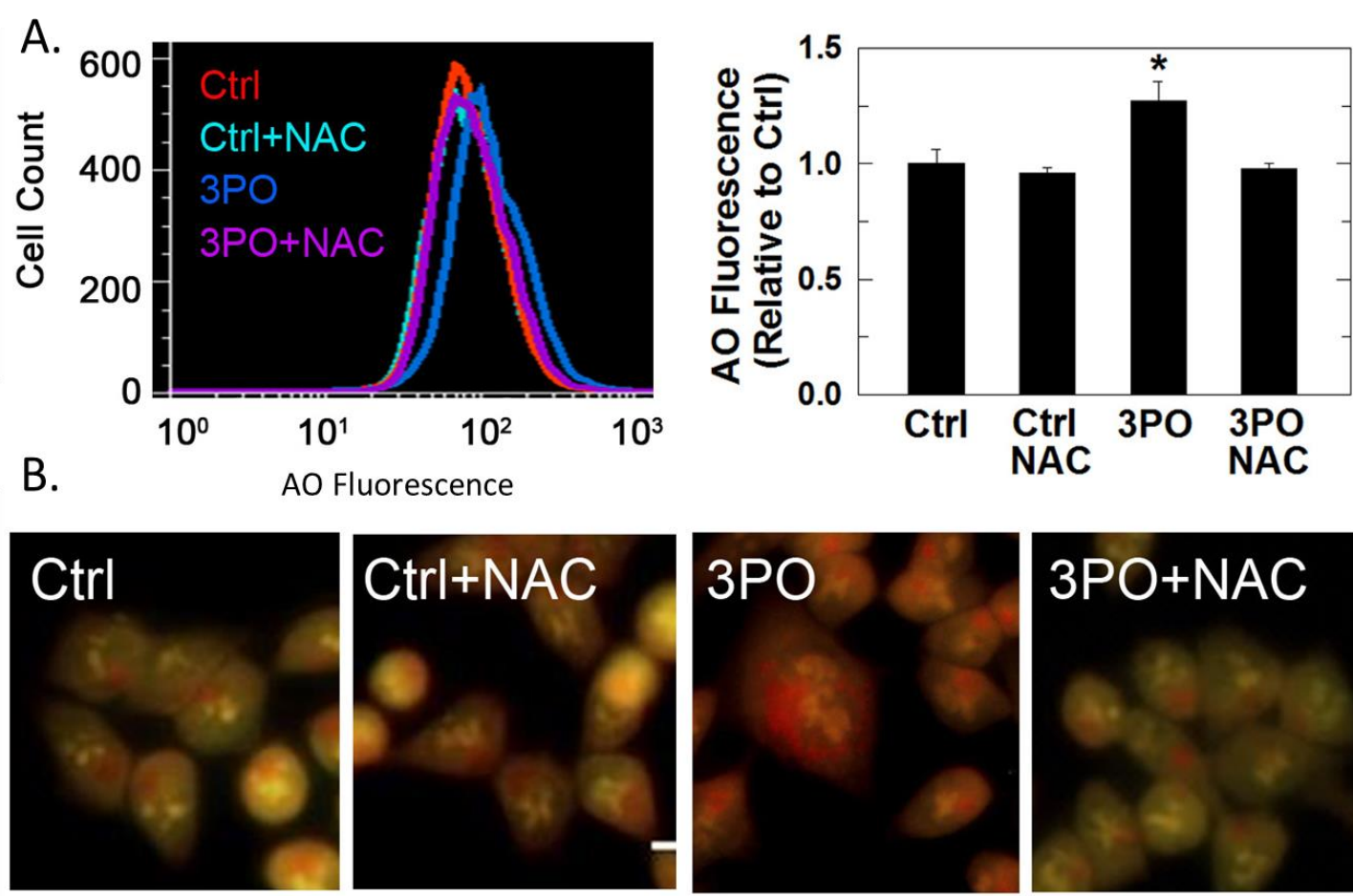

$3 \mathrm{PO}+\mathrm{NAC}$ 
Figure 23: $\mathrm{N}$-acetylcysteine rescues cells from death after treatment with 3PO. Cell death was measured in HCT-116 cells treated with either vehicle or 10 $\mu \mathrm{M} 3 \mathrm{PO} \pm 1 \mathrm{mM}$ NAC. Annexin-V and PI stained cells were collected using flow cytometry (Figure 23A). The percentage of cells with either annexin- $\mathrm{V}$ only staining or annexin-V/PI staining were determined and expressed as a percentage relative to control \pm SD from three experiments (Figure 23B, $C)$. $(p$ value $<0.05$ ) 
Figure 23.
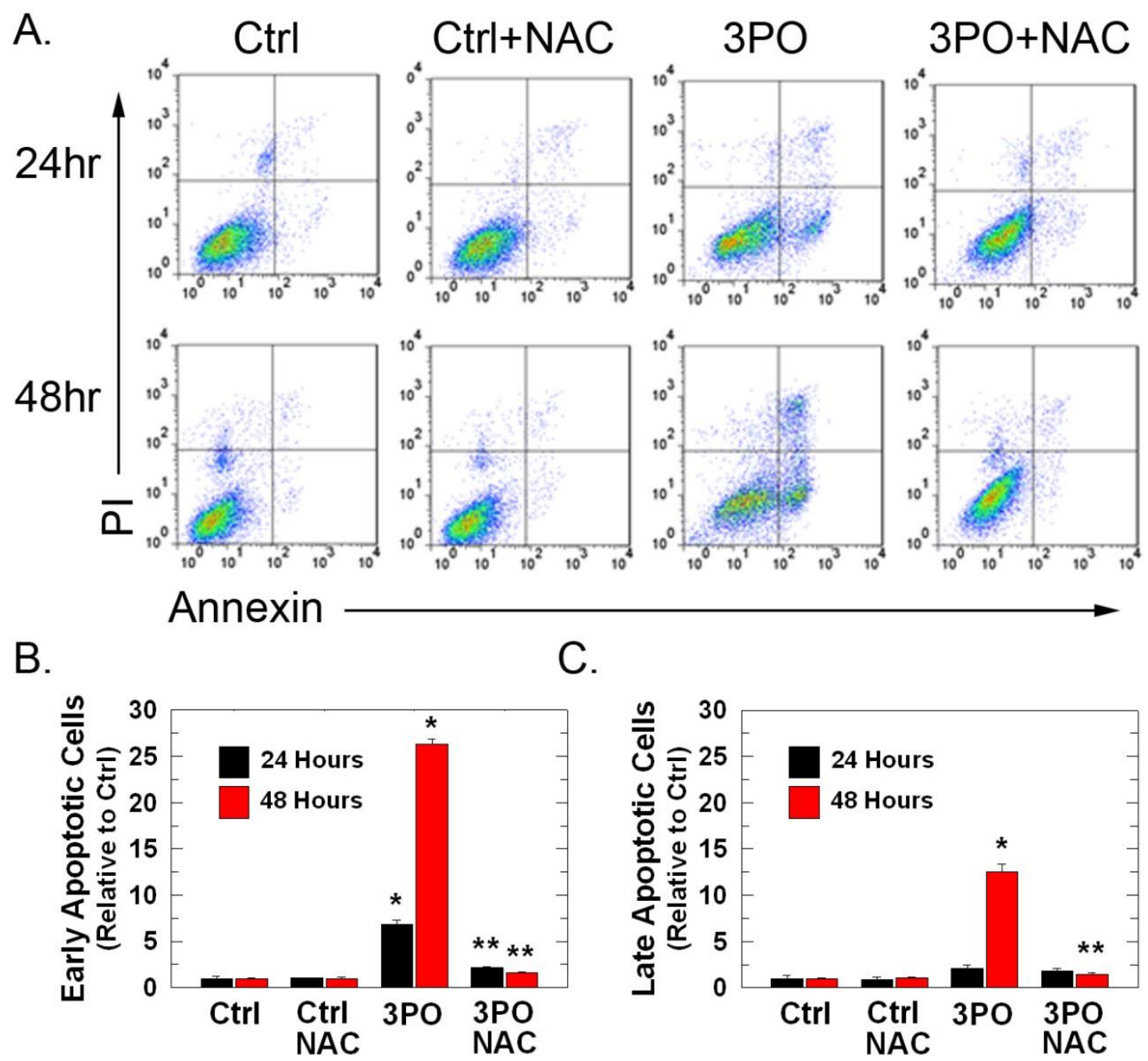


\section{Discussion}

The observation that PFKFB3 is overexpressed in neoplastic relative to normal tissues prompted the concept that targeting PFKFB3 expression may be a useful anti-cancer approach. Genetic manipulation is commonly used as a starting point to understand the importance of specific genes and proteins. Knockdown of PFKFB3 using gene silencing resulted in enzyme inhibition. However, as presented in Chapter II of this dissertation, knockdown of PFKFB3 also led to increased autophagic flux, presumably as a resistance mechanism to the knockdown. To expand these findings in a way that is more relevant and applicable to treating human disease, a drug-based approach was employed.

A drug that had previously been shown to inhibit PFKFB3, 3PO, was used to treat HCT-116 cells. Similar to PFKFB3 knockdown, PFKFB3 inhibition using $3 \mathrm{PO}$ reduced glucose uptake as well as reduced the levels of $\mathrm{F} 2,6 \mathrm{BP}$ in this model system. Corresponding to the changes seen after PFKFB3 knockdown, inhibition with 3PO also resulted in increased autophagic flux, as measured using protein markers, acridine orange staining and electron microscopy.

Knockdown of PFKFB3 resulted in decreased ATP levels and an increase in AMPK activation as measured by phosphorylation. Since knockdown usually 
results in a gradual change in protein expression and drug treatment has a more immediate effect it was not surprising that $3 \mathrm{PO}$ did not seem to activate AMPK as was seen with PFKFB3 knockdown. In fact, ATP levels did not even begin to decrease after 3PO treatment until at least 24 hours. Still, phosphorylated protein markers downstream of mTOR, phospho-p70S6K and phosphoribosomal protein S6 were found to be decreased even in the absence of AMPK activation. This indicated that for the acute response, an alternative mechanism was involved in mTOR suppression and autophagy activation. Autophagy induction, even that induced by metabolic stress, has been shown to occur independently of ATP and AMPK as the result of other stimuli such as endoplasmic reticulum (ER) stress or through SIRT1/FOX01/3 [126-128]. Additionally, starvation can result in decreased glutathione and the accumulation of reactive oxygen species [125, 129], and this oxidative stress is another stimulus for autophagic induction which can serve as a survival mechanism, mitigating damage by degrading oxidized proteins and by degrading damaged mitochondria [36-38, 130-138] There is abundant evidence that oxidative stressinduced autophagy occurs through inhibition of the mTOR pathway [125, 131, 139-142].

Oxidative stress is recognized as a potent activator of autophagy and can also be increased under stressful stimuli such as glucose starvation $[125,141$, 143]. Nutrient deprivation can lead to oxidative stress due to the accumulation of reactive oxygen species (ROS). ROS, reactive molecules that contain oxygen, are produced as a byproduct of normal cellular metabolism, are present at low 
levels in all cells, serving as signaling molecules [144] and playing a role in immune function $[145,146]$. However, when improperly regulated, ROS can cause damage to intracellular macromolecules and organelles [147]. The main defense against ROS is the action of anti-oxidants, including catalases, superoxide dismutases, and glutathione. Glutathione (GSH), a tripeptide formed by glycine, cysteine and glutamate, is the most abundant intracellular non-protein thiol and plays a critical role in limiting oxidative damage by scavenging ROS. Glutamylcysteine synthetase initiates the formation of GSH by catalyzing an amide linkage between glutamate and cysteine. Glycine is then added by GSH synthetase to complete the formation of GSH. Donation of electrons to ROS leads to the formation of an oxidized thiol radical (GS•) which can then form a disulfide bond with another thiol radical to form GSSG. Accumulation of GSSG can be toxic and normally accounts for about $1 \%$ of the total cellular glutathione [148] GSSG can be converted back to GSH by glutathione reductase, which requires reduced nicotinamide adenine dinucleotide phosphate (NADPH) as an electron donor. GSSG can also be transported across the plasma membrane as a way to prevent accumulation [149]. GSH may also have ROS-independent protective effects on cell function, some of which may be due to its function in glutathionylation [150-152]. Data presented here indicate that 3PO treatment results in a rapid decline in GSH as well as ROS accumulation.

Autophagic induction by 3PO seems to depend on oxidative stress, as supported by rescue using $\mathrm{N}$-acetylcysteine. $\mathrm{N}$-acetylcysteine, a compound with a reactive thiol group capable of reducing free radicals and a precursor to 
glutathione, rescues not only the oxidative stress caused by $3 \mathrm{PO}$, seen as a return in GSH and ROS levels towards that seen with vehicle treatment, but also prevents autophagic induction. At this time, the mechanism by which 3PO leads to an increase in oxidative stress, whether by increasing ROS which results in depletion of GSH, or by first causing depletion in the GSH pool resulting in the accumulation in ROS, is unclear. However, the induction of autophagy by the PFKFB3 inhibitor 3PO does appear to depend on oxidative stress and there is evidence that it may be through inhibition of mTOR, a pathway noted to be involved in autophagic induction by oxidative stress $[153,154]$. While $3 P O$ has mostly cytostatic effects at low doses, cytotoxicity is observed at high doses or after long periods of exposure. This cytotoxicity is almost completely abrogated upon the addition of NAC suggesting that inducing oxidative stress is a mechanism by with 3PO can result in cell death. However, the larger percentage of cells that survive treatment with $3 \mathrm{PO}$ suggests that these cells could be responding to the oxidative stress in order to prevent cell death. Autophagy is a well-known defense against oxidative stress $[138,155]$. Thus, inhibiting autophagy in the setting of 3PO treatment could shift this resistant population towards cell death.

Although through two distinct biochemical mechanisms, autophagy was induced by both the knockdown of PFKFB3 using siRNA and by enzyme inhibition using 3PO. The interest in PFKFB3 inhibitors as a new cancer treatment option means that recognition of potential drug resistance mechanisms may lead to more successful combinations when resistance in encountered. 
Autophagy is now a widely recognized process induced by stimuli such as nutrient deprivation or chemotherapeutic drugs. However, there is some disagreement as to the function of autophagy as a tumor suppressive versus as a tumor survival mechanism. In support of a tumor suppressive mechanism, the mammalian orthologue of yeast Atg6, Beclin1, is deleted in large number of human cancers including breast, ovarian and prostate cancers [156]. In this regard, autophagy is theorized to reduce genomic instability. Loss of this process, by deletion of Beclin1, results in tumorigenesis due to increased genomic instability. Additionally, autophagy has been seen to prevent necrotic inflammation which prevents the release of tumor promoting cytokines [90] and the persistent activation of autophagy can eventually lead to death via a caspase-independent mechanism [157]. The oncogenic regulation of autophagy is also not entirely clear. Some tumor suppressors (e.g. PTEN) activate autophagy, and some oncogenes can inhibit autophagy (Akt, Ras, ERK) while others, such as p53, have been shown to both activate and inhibit depending on the context [158-162]. Still, despite these indications that autophagy may suppress tumor initiation or growth, there is more evidence that autophagy is actually a tumor survival mechanism. In order to metastasize, tumor cells must overcome matrix detachment-induced cell death, anoikis. Autophagy has been shown to be protective against cell death during this process which may function in facilitating metastatic spread [163]. Autophagy can also serve to promote survival of dormant tumor cells which could have major implications for adjuvant therapies aimed at preventing recurrence. [164, 
165]. This role of autophagy in dormancy is the result of reduced proliferation, the same function that is also touted as a tumor suppressive function. However, the function to reduce proliferation until adequate nutrients or a more conducive environment are encountered is surely one that can be viewed as tumorigenic rather than suppressive. Additionally, there is now considerable evidence that the combination of inhibitors of autophagy with drugs that induce autophagy in cancer cells can increase tumor cell death $[77,158,166-168]$

The most currently accepted model of autophagy is that of a process with a dual nature, depending on tumor type and stage. Autophagy may suppress tumor initiation via reduced genomic instability and prevention of chronic inflammation and necrosis, while later serving as a cell survival mechanism by allowing stress tolerance through increased production of metabolic precursors, mitigating excessive damage that can be caused by damaged mitochondria, and facilitating metastasis by protecting from anoikis-induced cell death.

To determine if the autophagy induced by knockdown or inhibition of PFKFB3 could be exploited to increase efficacy as an anti-tumor agent, the next step was to evaluate how tumor cells reacted to the combination of PFKFB3 inhibition and autophagy inhibition both in vitro and in vivo. 


\section{CHAPTER IV}

\section{INHIBITION OF PFKFB3 HAS ADDITIVE ANTI-TUMOR EFFECTS WITH PHARMACOLOGIC AUTOPHAGY INHIBITORS}

\section{Introduction}

While there was an indication of cytotoxic activity of 3PO in transformed cells, this toxicity occurred at concentrations that also had toxic effects on untransformed cells. At lower concentrations, when the drug was found to influence transformed cells more than normal cells, the effect was more cytostatic [53]. In vivo tumor experiments using 3PO resulted in a significant reduction in tumor growth relative to vehicle treated animals. However, tumors in animals treated with $3 \mathrm{PO}$, while smaller than the controls, continued to grow through drug treatment. Like most drugs currently used to treat human cancers, 3PO seemed to have restricted efficacy as a single drug option, making rational drug combination an important step to ensure maximal efficacy when used to treat human cancer patients.

The majority of cancers not amenable to surgical removal are treated with combinations of chemotherapeutic drugs. It is well known that neoplastic cells are capable of evading and resisting treatment making the recognition of 
resistance mechanisms imperative to the identification of successful combinations of drugs to counter such defenses. There are numerous mechanisms employed by tumor cells to avoid death caused by chemotherapeutic agents. In some tumors, cells have membrane protein pumps that prevent drug from accumulating inside [149, 169]. Other mechanisms involve changes that reduce the sensitivity to drug-induced apoptosis, allowing for survival even in the presence of pro-apoptotic signals [170]. Another way that tumor cells resist destruction is through the activation of autophagy, a ubiquitous intracellular catabolic processing pathway [41-44]. An increase in autophagic processing results in the degradation of intracellular proteins and organelles for the production of biosynthetic precursors can be used to fuel the continued production of ATP and lead to cell survival under adverse conditions. Autophagy is now recognized as a major resistance mechanism of tumors to a large number of drug therapies $[50-52,55,57,78]$. The cause for increased autophagic flux is not always identified but, nonetheless, can be taken advantage of by the addition of autophagy inhibitors to therapy regimens.

The increased detection of autophagy due to drug treatment in cancer has resulted in great interest in the use of autophagy inhibitors in cancer therapy. While there are now a number of drugs known to inhibit autophagy, the drug that is currently the most widely used for this purpose is chloroquine, a drug that was originally developed as an anti-malarial agent.

Chloroquine is a 4-aminoquinoline drug that has been used to treat malaria for more than half a century. The original observation that malaria could 
be treated using the bark of cinchona trees led to the isolation of quinine. The molecular structure of quinine was determined and later, a derivative with potent anti-malarial activity called Resochin, N'-(7-chloroquinolin-4-yl)-N,N-diethylpentane-1,4-diamine, and later renamed chloroquine, was produced [171, 172]. Chloroquine in an unprotonated form can diffuse across membrane bilayers. However, once protonated in the lysosome, chloroquine is no longer able to exit and accumulates resulting in the inhibition of lysosomal enzymes such as phospholipase A2, lysophospholipid acylhydrolase [173, 174]. This inhibition blocks the final step of autophagy, degradation on autophagosomal contents by lysosomal enzymes. Chloroquine was later shown to be effective in treating other diseases such as rheumatoid arthritis and HIV [175-177]. The observation of a reduced incidence of Burkitt's lymphoma in malaria patients treated with chloroquine was made in 1989 [178]. More recently, chloroquine has been shown to reduce autophagy in vivo and to potentiate the anti-cancer effects of some cancer therapies $[55,167,179,180]$. The relatively mild safety profile and widely accepted use of chloroquine makes this compound attractive for use in a combination drug trial for cancer. As such, chloroquine is currently being used in combination with a number of other agents as part of clinical trials, including carmustine, paclitacel, gefitinib, and temozolomide among others reviewed in [181].

To determine the effect of autophagy inhibition in combination with PFKFB3 inhibition in transformed cells, HCT-116 cells were treated with combinations of PFKFB3 inhibitors and autophagy inhibitors and cell death was 
measured. The addition of autophagy inhibitors to PFKFB3 inhibitors resulted in more cell death than either inhibitor alone. While these results were encouraging, in vitro assays do not always accurately predict the effects of certain treatments when used in vivo. This can be due to a number of factors including the tumor microenvironment, such as access to blood supply and nutrients, or to the heterogeneous nature of tumors. Additionally, systemic effects such as drug metabolism or activation/inactivation and unexpected toxicities are impossible to predict using an in vitro modeling system. As predicted, tumors from animals treated with the combination of $3 \mathrm{PO}$ and chloroquine grew significantly slower relative to animals treated with either drug alone. While a significant amount of work needs to be done before these results can translate into an effective treatment option for human cancer patients, data here support the increased efficacy of PFKFB3 inhibitors when combined with inhibitors of autophagy. 


\section{Materials and Methods}

\section{$\underline{\text { Cell Culture }}$}

Human colorectal carcinoma cells (HCT116) were cultured as described in

Chapter II. Lewis lung carcinoma cells (LLC) obtained from American Type Culture Collection (Manassas, VA) were cultured in Dulbecco's Modified Eagle Medium (Gibco, Grand Island, NY) supplemented with 10\% calf serum and $50 \mathrm{ug} / \mathrm{mL}$ gentamicin. Cells were incubated at $37^{\circ} \mathrm{C}$ with $5 \% \mathrm{CO} 2$.

\section{Drug Treatment}

3-(3-pyridinyl)-1-(4-pyridinyl)-2-propen-1-one (3PO), PF15, PF158 (Advanced Cancer Therapeutics, Louisville, KY). Chloroquine, 3-methyladenine, Spautin-1, Bafilomycin A1 from (Sigma, St. Louis, MO). Samples in which bafilomycin A1 was used were treated with 1nM bafilomycin A1 24 hours prior to harvest.

\section{Protein Extraction and Immunoblotting}

Protein extraction and immunoblotting were performed as described in Chapter II. 


\section{Acridine Orange Immunofluorescence}

Acridine orange staining and immunofluorescence measurements were performed as described in Chapter II.

\section{Flow Cytometry for Cell Death}

Cell death was assessed using method described in Chapter II.

\section{$\underline{\text { Tumor model system }}$}

12 week old female C57/BL6 mice were injected subcutaneously with $1 \times 10^{6}$ Lewis lung carcinoma (LLC) cells and once tumors reached 150-200 mg, mice were randomized into four groups ( $N=6$ per group): Group 1: Vehicle (DMSO+PBS), Group 2: Chloroquine (DMSO+50mg/kg CQ), Group 3: $3 P O$ (0.07mg/g 3PO+PBS), Group 4: (0.07mg/g 3PO+50mg/kg CQ). Drug treatments were based on published tumor models [53, 167, 182]. Mice were given two daily intraperitoneal injections with either vehicle or drug and tumors were measured using calipers for estimation of tumor volume. At the conclusion of the study, mice were euthanized and tumors were removed. Tumors tissues were fixed in paraformaldehyde and prepared for immunohistochemistry. 


\section{Immunohistochemistry}

Tumors excised after completion of all tumor measurements were fixed in paraformaldehyde for 24 hours and then embedded in paraffin, sections and stained with anti-cleaved caspase 3 antibody using standard immunohistochemical methods. 


\section{Results}

\section{Pharmacologic inhibition of autophagy in combination with 3PO increases tumor cell death}

To determine if autophagy following $3 \mathrm{PO}$ treatment serves as a survival mechanism, chloroquine, an agent that accumulates in lysosomes and interferes with autophagy was used in combination with 3PO. Treatment of HCT-116 cells with 15 or $30 \mu \mathrm{M}$ chloroquine led to a dose-dependent increase in cell death when combined with 3PO treatment as measured by flow cytometric analysis of annexin $\mathrm{V}$ and propidium iodide staining (Figure 24).

Two additional inhibitors of autophagy were also used in combination with 3PO to look for increased cell death, 3-methyladenine and Spautin-1. 3methyladenine (3MA), an inhibitor of type III phosphatidylinositol 3-kinases, which blocks the formation of autophagosomes was used to treat HCT-116 cells $(1 \mathrm{mM})$ that were also treated with either vehicle, or 3PO. Cell death was assessed using annexin V and PI staining and quantified by flow cytometry. The number of cells staining positive for both annexin $\mathrm{V}$ and PI was increased in samples treated with the combination of $3 \mathrm{MA}$ and $3 \mathrm{PO}$ relative to either drug alone (Figure 25). Spautin-1, which inhibits autophagy by promoting increased proteasomal degradation of class III PI3 kinase complexes through inhibition of 
ubiquitin-specific peptidases USP10 and USP13 also resulted in greater numbers of annexin- $\mathrm{V}$ and $\mathrm{PI}$ double positive cells when used in combination with 3PO (Figure 26).

To assess whether the combined effects of autophagy inhibitors and the PFKFB3 inhibitor was limited only to the specific inhibitor 3PO, two other derivatives of $3 \mathrm{PO}$ were used to treat cells in combination with the autophagy inhibitor chloroquine. Cell death, assessed using flow cytometry measuring annexin V and PI staining, was increased when chloroquine was combined with 3PO derivative PF-158 (Figure 27) or derivative PF-15 (Figure 28).

This increase in cell death upon the addition of chloroquine was also noted in HCT-116 cells that had been transfected with a siRNA directed against PFKFB3 (Figure 29). 
Figure 24: Synergistic increase in cell death when 3PO is combined with the autophagy inhibitor chloroquine. Annexin-V/PI staining was performed in cells treated with vehicle, 3PO, chloroquine, or the combination of $3 \mathrm{PO}$ and chloroquine for 48 hours. Quantitation of cell staining was performed using flow cytometry (Figure 24A) and the number of cells staining with both annexin- $\mathrm{V}$ and PI was quantitated and is expressed as the percentage relative to control $\pm \mathrm{SD}$ from three experiments (Figure $24 \mathrm{~B}) .(p$ value $<0.05)$ 
Figure 24.

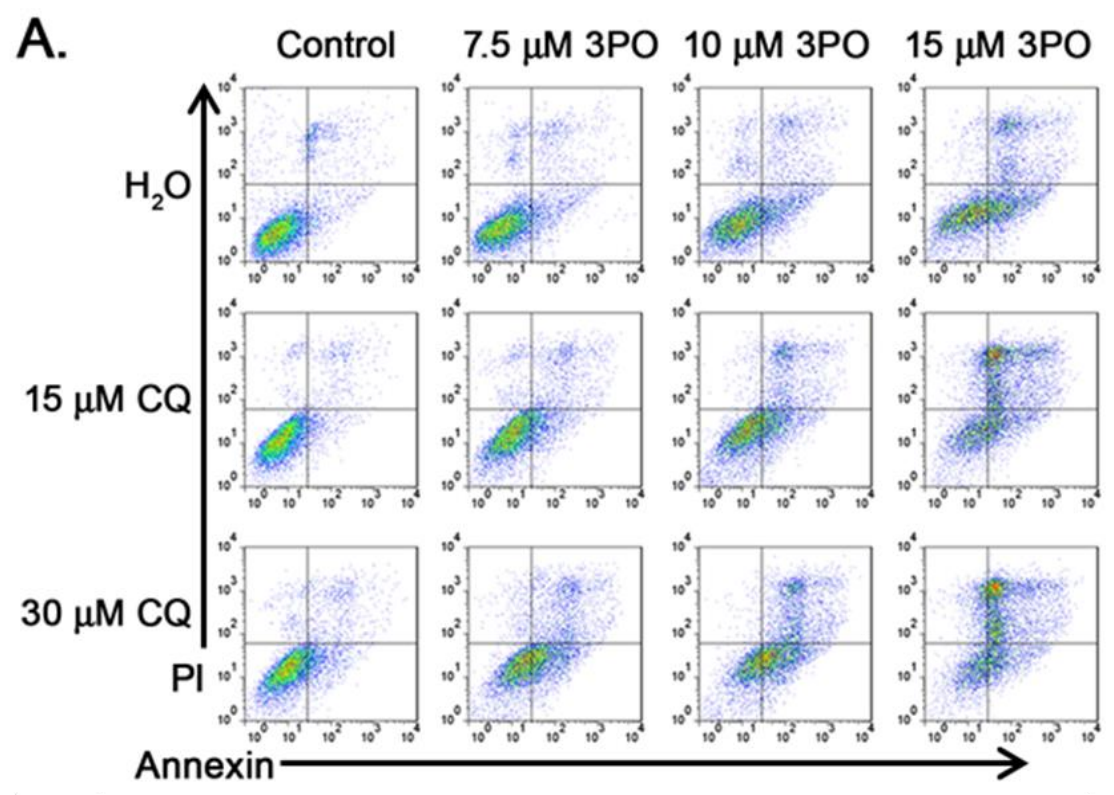

B.

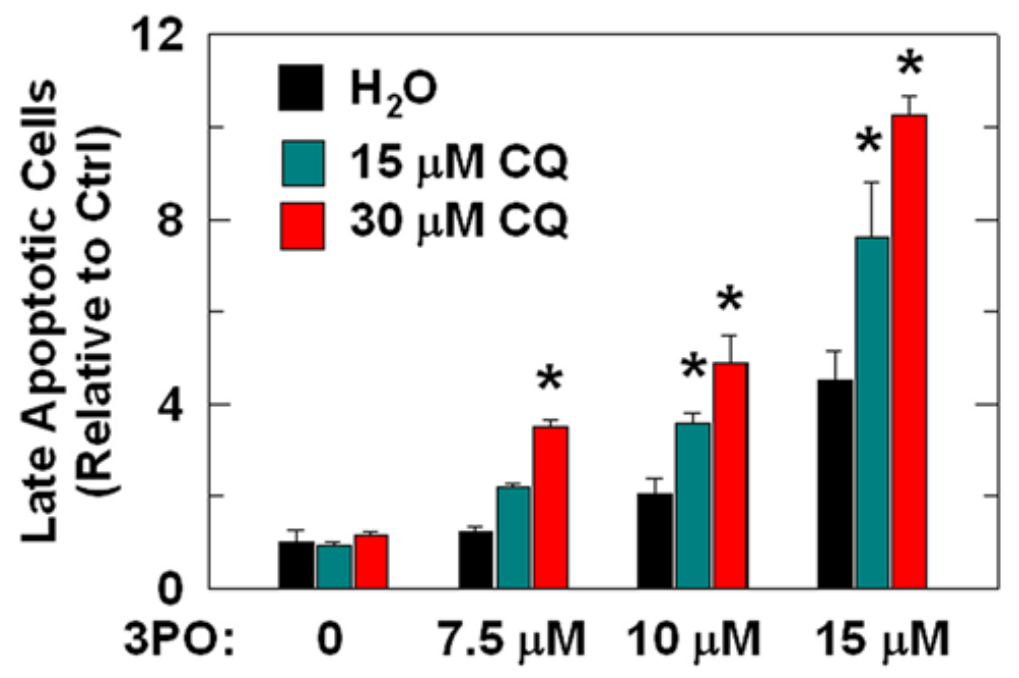


Figure 25: The autophagy inhibitor, 3-methyladenine, increased cell death when combined with 3PO. HCT-116 cells were treated with either vehicle or $7.5 \mu \mathrm{M}$ or $10 \mu \mathrm{M} 3 \mathrm{PO} \pm 1 \mathrm{mM} 3$-methyladenine (3MA). 24 and 48 hours after treatment cells were stained with annexin- $\mathrm{V}$ and $\mathrm{PI}$ and measured using flow cytometry (Figure 25A). Cell death was quantitated as the percentage of cells staining positive for annexin- $\mathrm{V}$ and $\mathrm{PI}$ relative to control $\pm \mathrm{SD}$ from three experiments (Figure 25B). ( $p$ value $<0.05)$ 
Figure 25.

A.

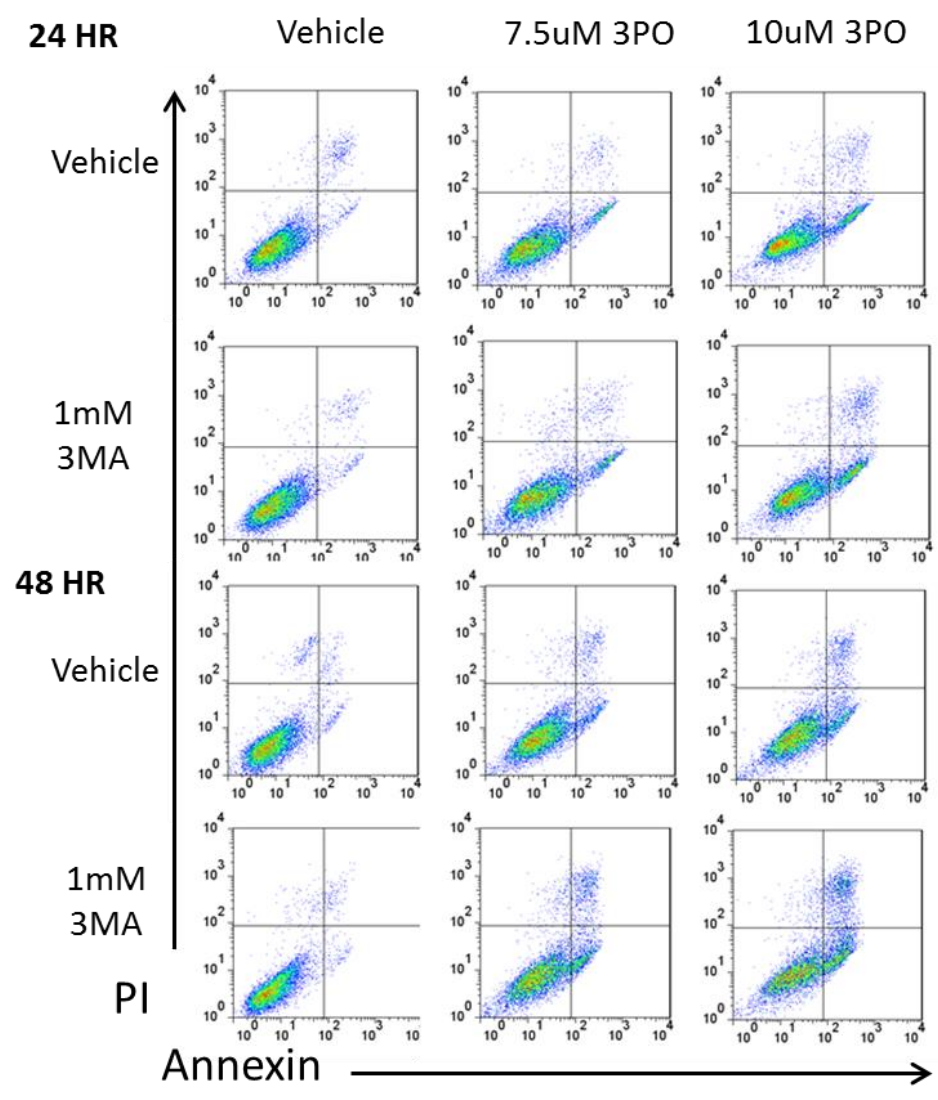

B.

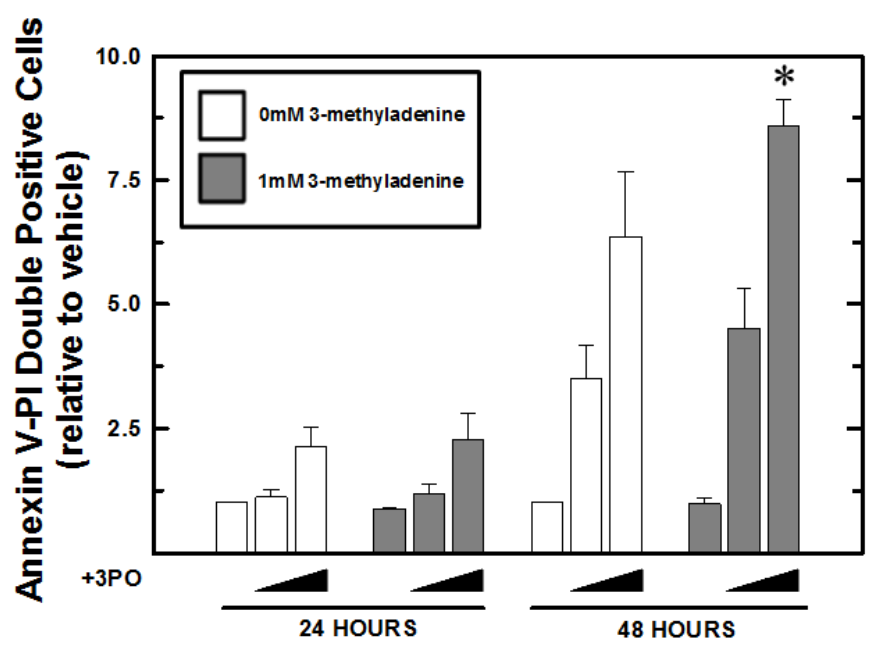


Figure 26. The combination of Spautin-1 and 3PO increased cell death. HCT-116 cells were treated with either vehicle, 7.5 or $10 \mu \mathrm{M} 3 \mathrm{PO} \pm 15 \mu \mathrm{M}$ Spautin-1. After 24 or 48 hours of treatment cells were stained with annexin-V and $\mathrm{PI}$ and collected by flow cytometry (Figure 26A). The percentage of cells stained positive for both annexin- $\mathrm{V}$ and $\mathrm{PI}$ was quantitated and is expressed relative to control \pm SD from three experiments (Figure $26 \mathrm{~B}) .(p$ value $<0.05)$ 
Figure 26.

A.

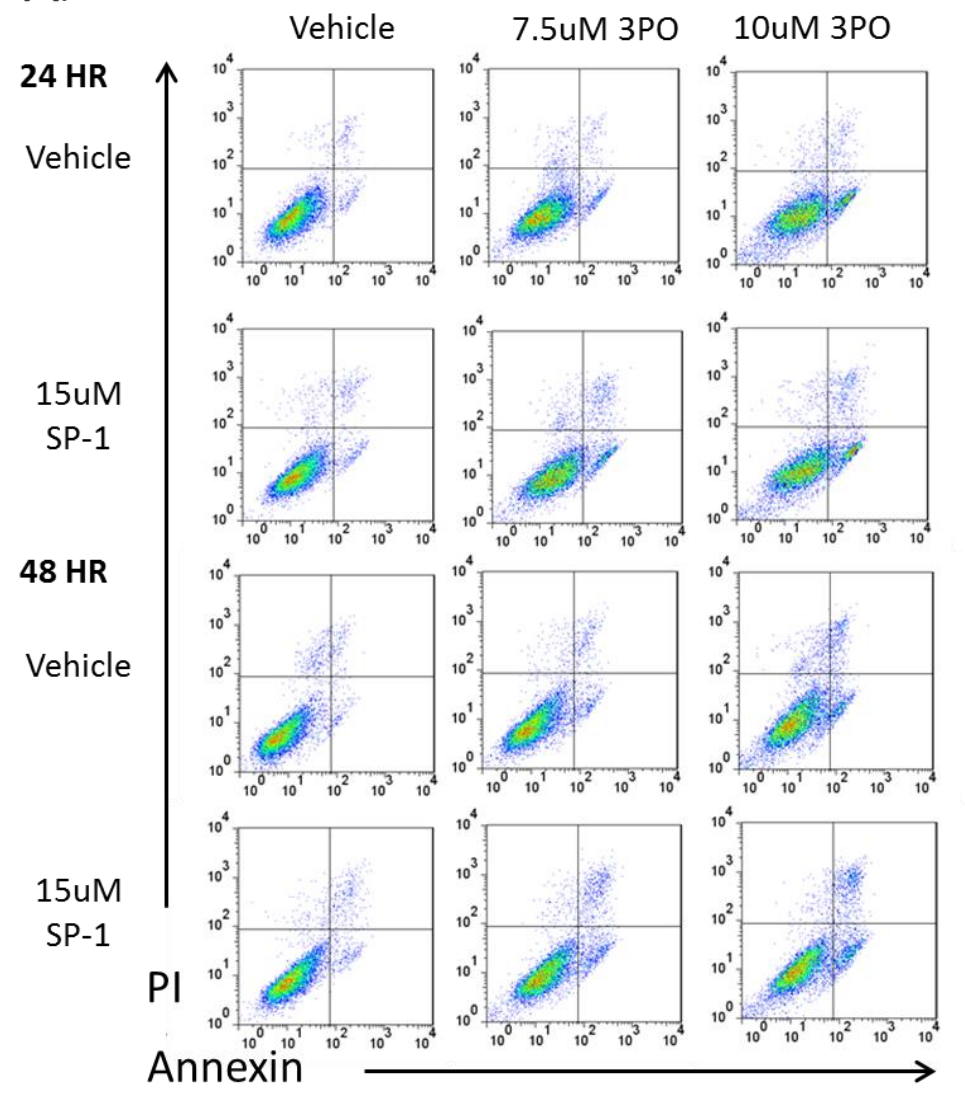

B.

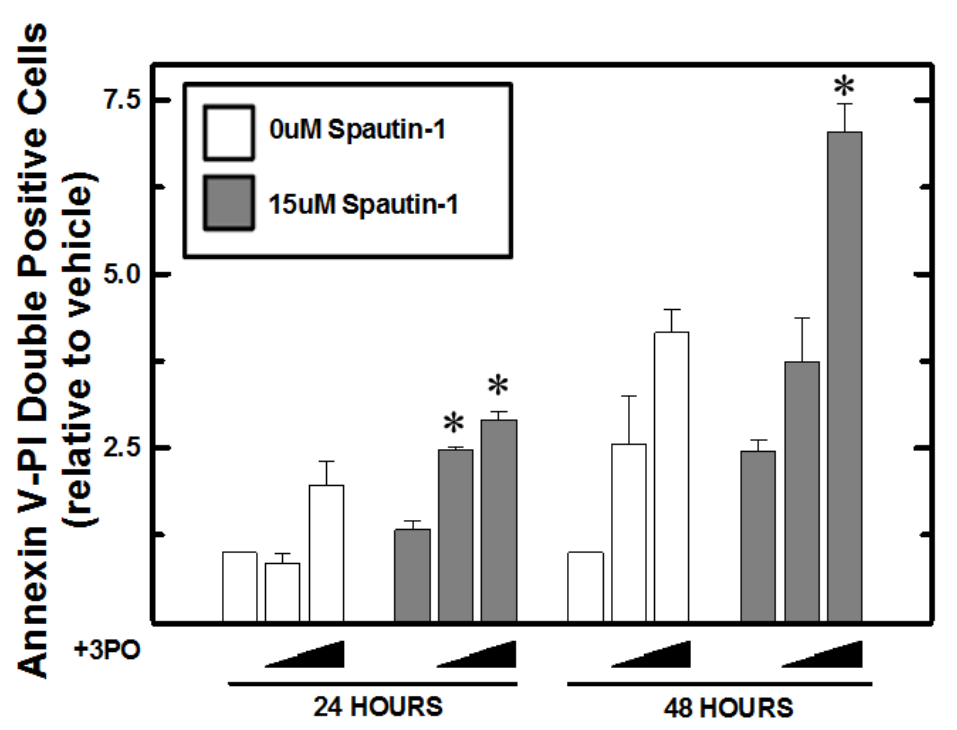


Figure 27. The 3PO derivative PF158 causes increased cell death when combined with chloroquine. HCT-116 cells treated with vehicle or $0.5 \mu \mathrm{M}$ $\mathrm{PF} 158 \pm 30 \mu \mathrm{M} \mathrm{CQ}$ were stained with annexin-V and PI and collected by flow cytometry (Figure 27A). Annexin-V/PI positive cells were quantitated as percentage of total relative to control \pm SD from three experiments (Figure 27B). $(p$ value $<0.05)$ 
Figure 27.
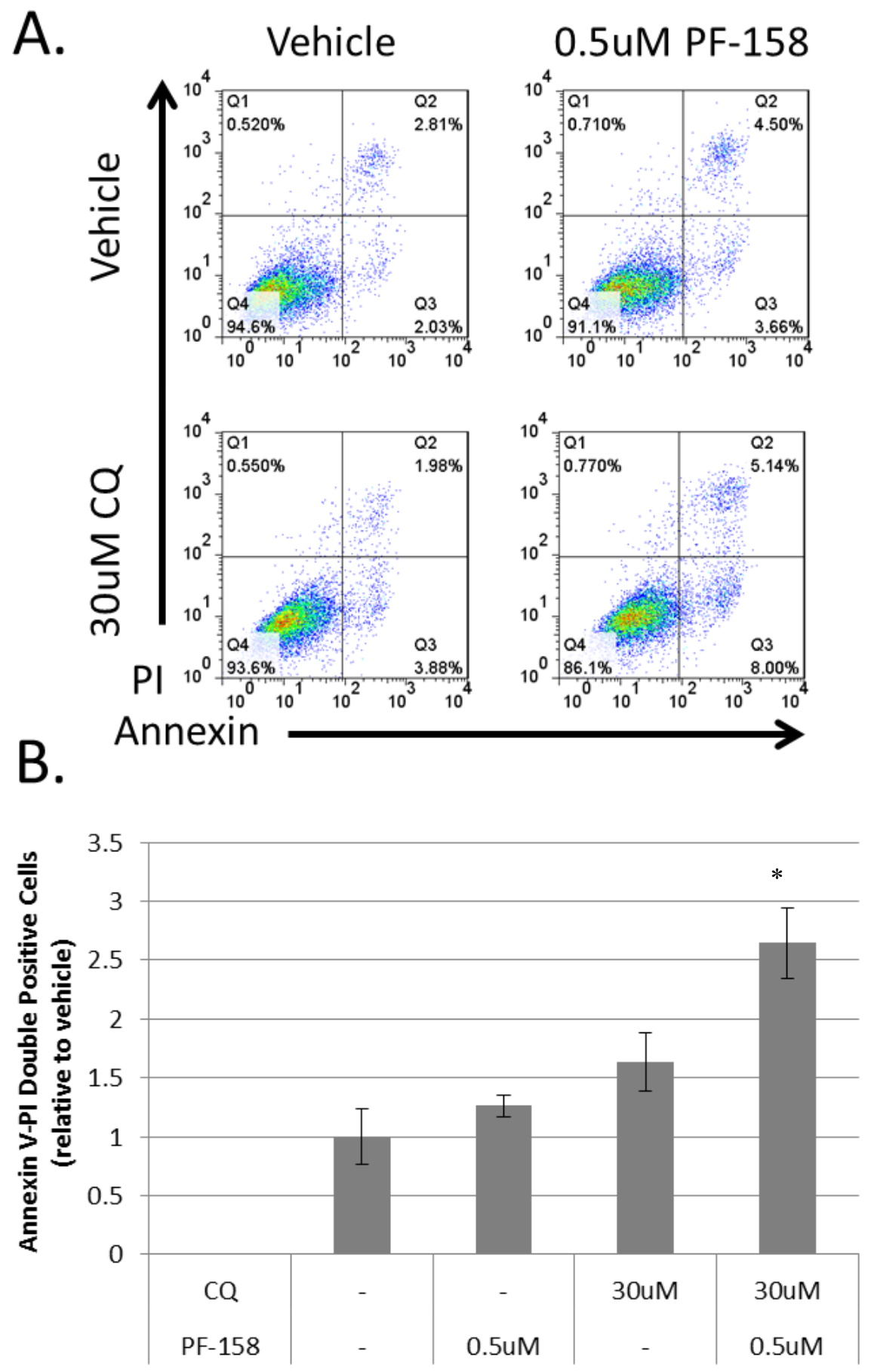
Figure 28. Cell death by PF-15, a derivative of $3 \mathrm{PO}$, is increased when combined with chloroquine. After treatment with either vehicle or $1 \mu \mathrm{M} P F-15 \pm$ $30 \mu \mathrm{M}$ chloroquine, HCT-116 cells were stained with annexin-V and PI and collected by flow cytometry (Figure 28A) (Figure 28A). Cells staining positive for annexin- $\mathrm{V}$ and $\mathrm{PI}$ were quantitated as percentage of total relative to control presented as mean \pm SD from three experiments (Figure $28 B)$. ( $p$ value $<0.05)$ 
Figure 28.

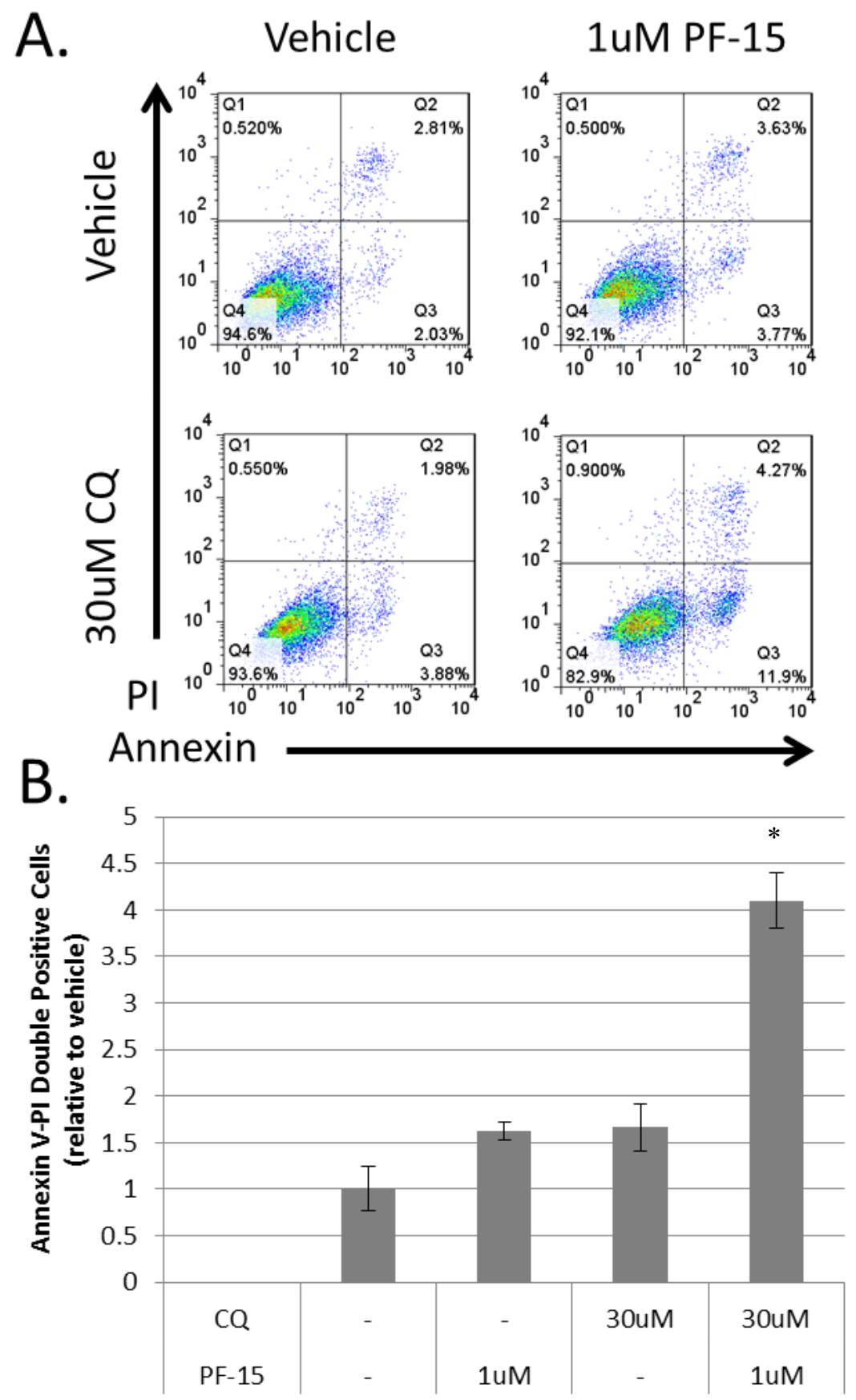


Figure 29: Cell death due to knockdown of PFKFB3 is increased when combined with the autophagy inhibitor chloroquine. HCT-116 cells were transfected with either a negative control siRNA or with a PFKFB3-specific siRNA $\pm 30 \mu \mathrm{M} \mathrm{CQ}$. After 48 hours of transfection, cells were stained with annexin-V and PI and measured using flow cytometry (Figure 29A). Quantitation of cells staining positive for annexin- $\mathrm{V}$ and $\mathrm{PI}$ was performed relative to control and is expressed as the mean \pm SD from three experiments (Figure 29B). ( $p$ value $<$ 0.05) 
Figure 29.

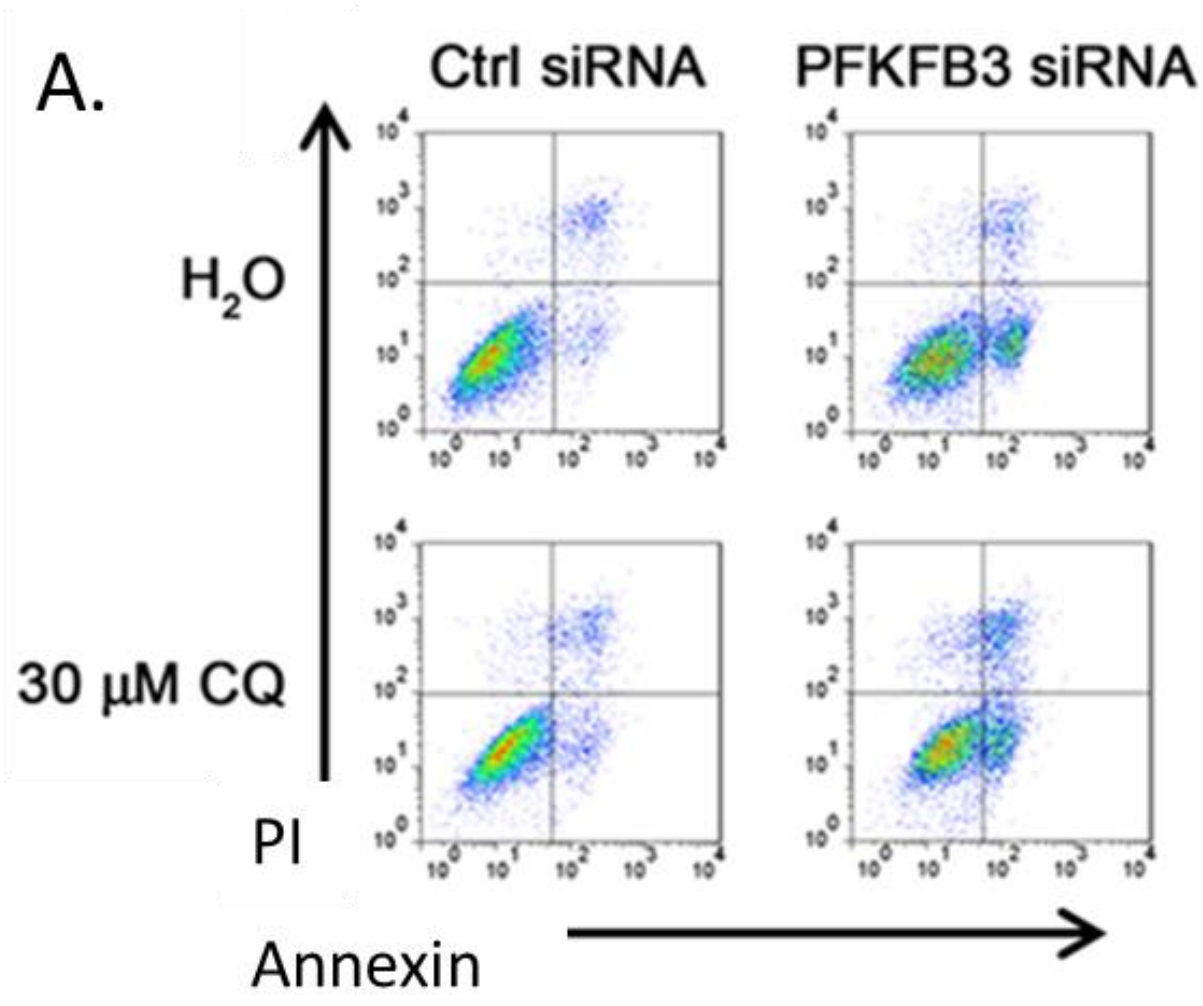

B.

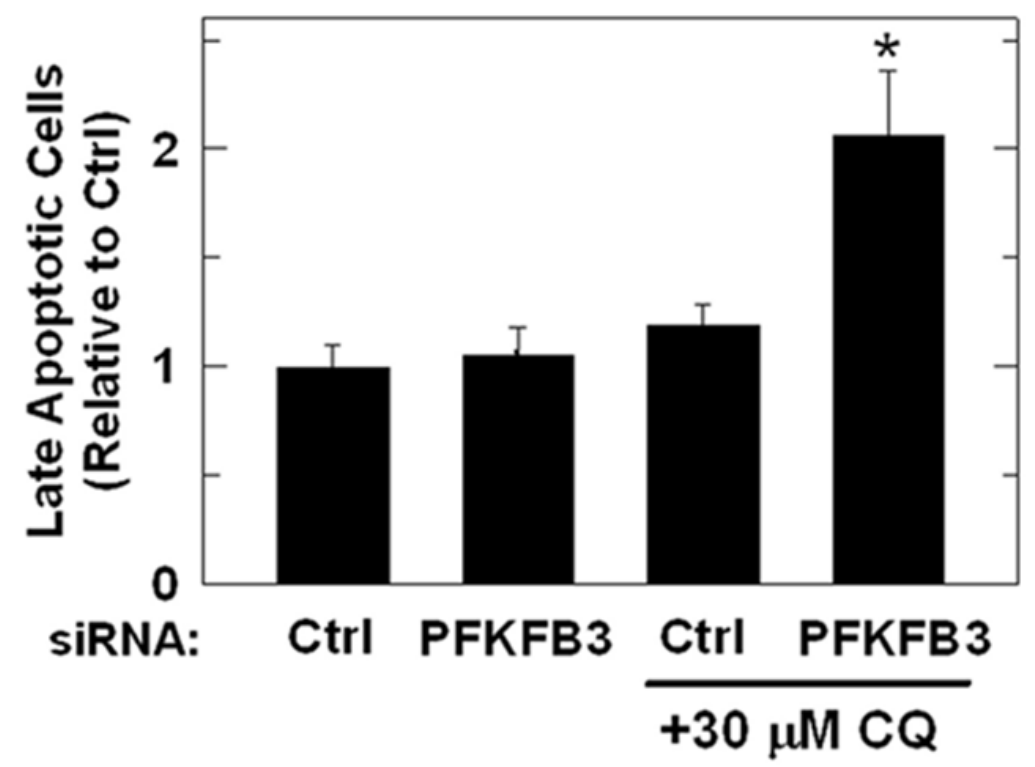




\section{Validation of a mouse tumor cell line prior to in vivo experiment}

Before moving to an in vivo model, a mouse tumor cell line, Lewis lung carcinoma (LLC) was evaluated for autophagy and increased sensitivity to chloroquine following treatment with 3PO. Lewis lung carcinoma cells treated with $10 \mu \mathrm{M}$ 3PO had increased levels of LC3-II relative to control. This increase was further enhanced upon the addition of bafilomycin A1 (Figure 30). Acridine orange immunofluorescence was also increased by $10 \mu \mathrm{M}$ 3PO in LLC cells as determined visually by fluorescence microscopy (Figure 31 A), and quantified by flow cytometry (Figure 31B). Similar to the HCT-116 cells, Lewis lung carcinoma cell death was also increased when chloroquine treatment was used in combination to 3PO treatment relative to either drug alone (Figure 32). 
Figure 30: Autophagic protein marker LC3-II is increased in Lewis lung carcinoma cells exposed to 3PO. LLC cells were treated with $25 \mu \mathrm{M} 3 \mathrm{PO}$ for 24 hours. LC3-II levels were measured using immunoblotting and quantitative densitometry was performed. Levels expressed as mean fold change LC3-II/ $\beta$ actin relative to control $\pm S D($ Figure $30 \mathrm{~B}) .(p$ value $<0.05)$ 
Figure 30.

A.

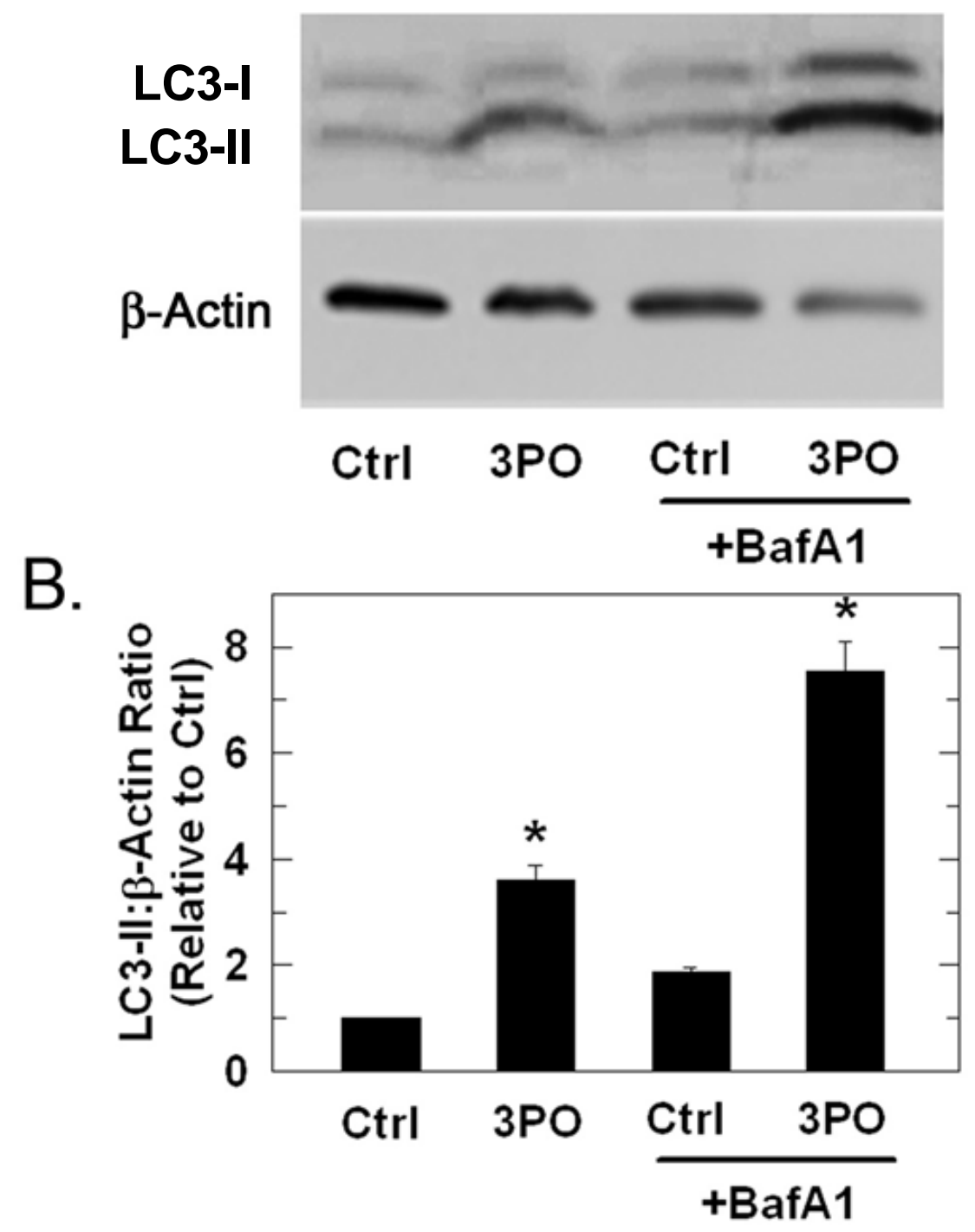


Figure 31: Lewis lung carcinoma cells treated with 3PO have increased acridine orange staining. LLC cells treated with $25 \mu \mathrm{M} 3 \mathrm{PO}$ for 24 hours were stained with acridine orange. Cells were viewed by fluorescent microscopy and representative images were taken (Figure 31A). Flow cytometry was performed and the relative number of cells with a high level of acridine orange staining was quantitated and is expressed as the mean \pm SD from three experiments (Figure 31B). $(p$ value $<0.05)$ 
Figure 31.
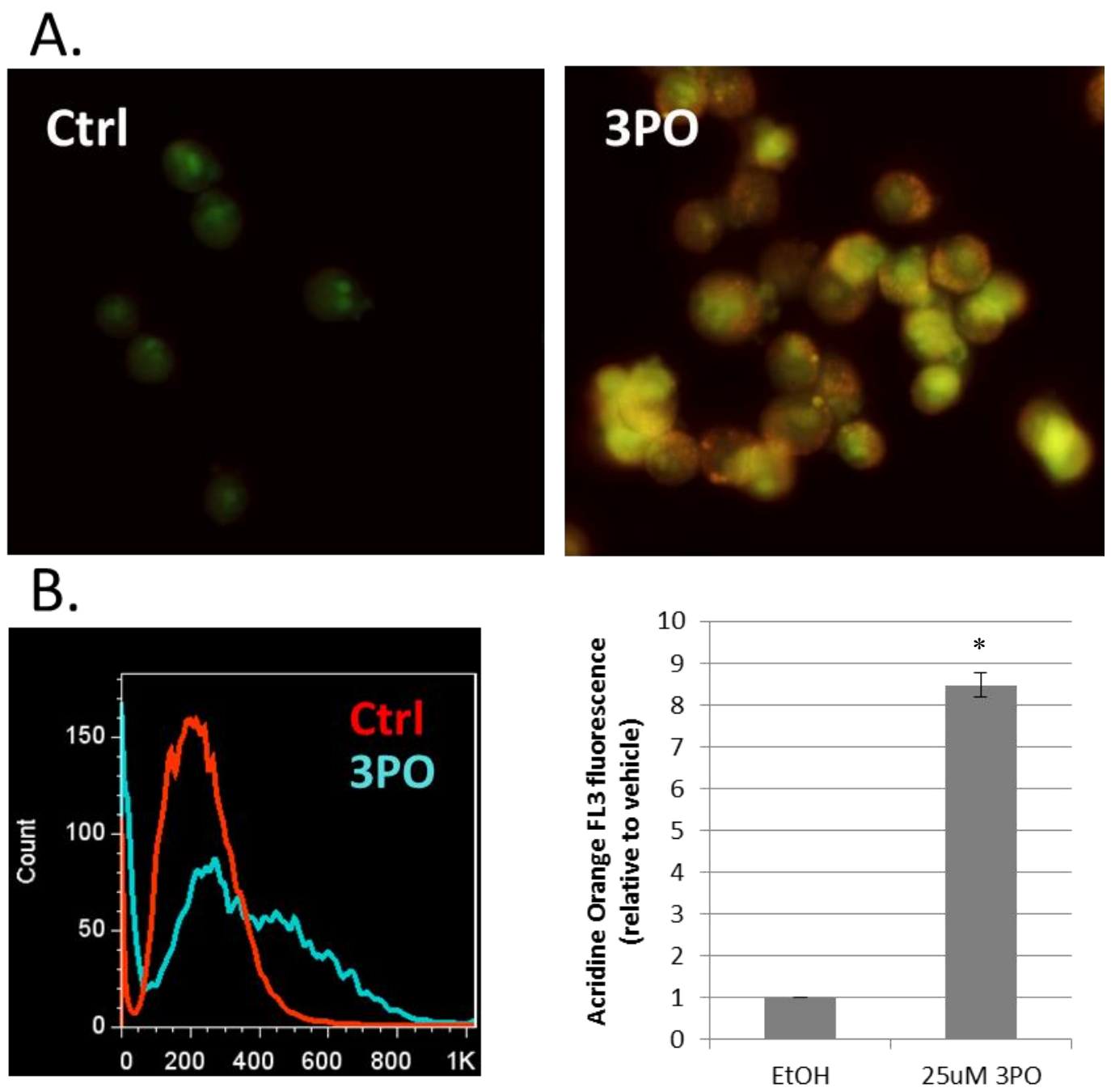
Figure 32: The combination of 3PO and chloroquine has synergistic effects on cell death in Lewis lung carcinoma cells. LLC cells were treated with either vehicle or $10 \mu \mathrm{M} 3 \mathrm{PO} \pm$ either 15 or $30 \mu \mathrm{M} \mathrm{CQ}$. After 24 hours of treatment cells were stained with annexin- $\mathrm{V}$ and $\mathrm{PI}$ and measured using flow cytometry (Figure 32A). Cells staining positive for both annexin-V and PI were quantitated as the percentage of the total relative to control and data is presented as the mean \pm SD from three experiments (Figure $32 \mathrm{~B}) .(p$ value $<0.05)$ 
Figure 32.
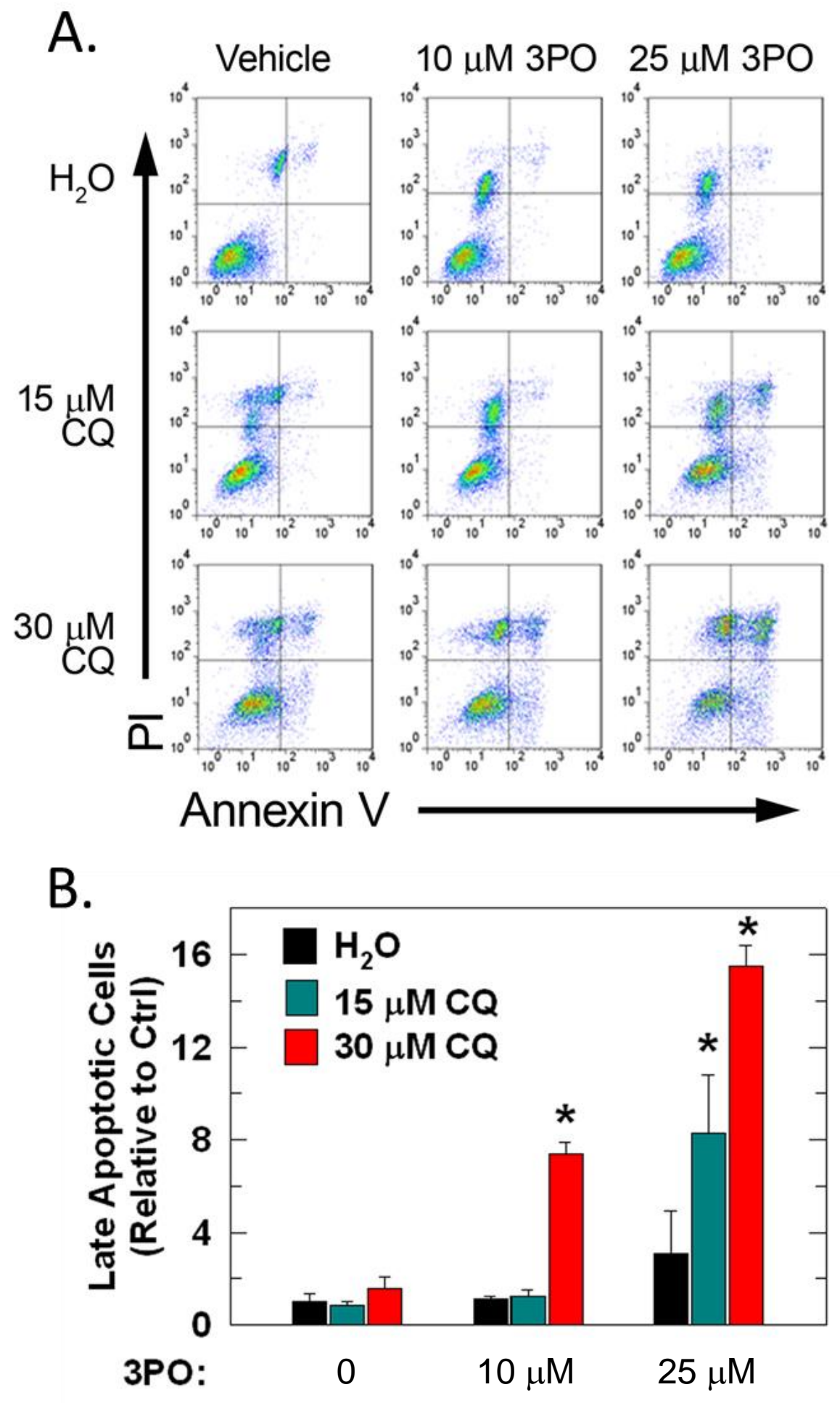


\section{Combination of autophagy inhibition and 3PO decreases tumor growth in}

vivo

12 week old female C57/BL6 mice were used to determine the in vivo effect of the combination of chloroquine and 3PO on tumor growth. Animals were injected subcutaneously with $1 \times 10^{6}$ Lewis lung carcinoma cells. When tumors reached $150-200 \mathrm{~mm}^{3}$, calculated based on volume, mice were randomized into four treatment groups ( $\mathrm{N}=6$ per group). Group 1: Vehicle (DMSO+PBS), Group 2: Chloroquine (DMSO+50mg/kg CQ), Group 3: 3PO (0.07mg/g 3PO+PBS), Group 4: $(0.07 \mathrm{mg} / \mathrm{g} 3 \mathrm{PO}+50 \mathrm{mg} / \mathrm{kg} \mathrm{CQ})$. Daily tumor measurements were obtained using calipers for estimation of tumor mass. The experiment was concluded two weeks from the start of drug treatment and tumors were collected for further examination. The tumor mass was significantly reduced in animals treated with both 3PO and chloroquine relative to either drug treatment alone (Figure 33). Excised tumors were fixed and prepared for immunohistochemistry. The tumor sections were stained with an antibody recognizing cleaved caspase-3, a key protein in the execution phase of apoptosis. Tumors from animals treated with the combination of 3PO and chloroquine were noted to have an increased number of cleaved caspase-3 positive cells relative to tumors from animals treated with either drug alone (Figure 34). 
Figure 33: Combination therapy using 3PO and chloroquine reduces tumor growth in a Lewis lung carcinoma tumor model in vivo. C57/BL6 mice were inoculated with $1 \times 10^{6}$ Lewis lung carcinoma cells by subcutaneous flank injection. Mice were randomized into 4 treatment groups when tumors reached $150-200 \mathrm{~mm}^{3}$ and were treated by i.p. injections with either vehicle, $50 \mathrm{mg} / \mathrm{kg}$ chloroquine, $0.07 \mathrm{mg} / \mathrm{g} 3 \mathrm{PO}$, or a combination of the two drugs. Tumor measurements taken over the course of treatment were used to calculate tumor mass. Data is presented as mean tumor mass \pm SD. ( $p$ value $<0.05)$ 
Figure 33.

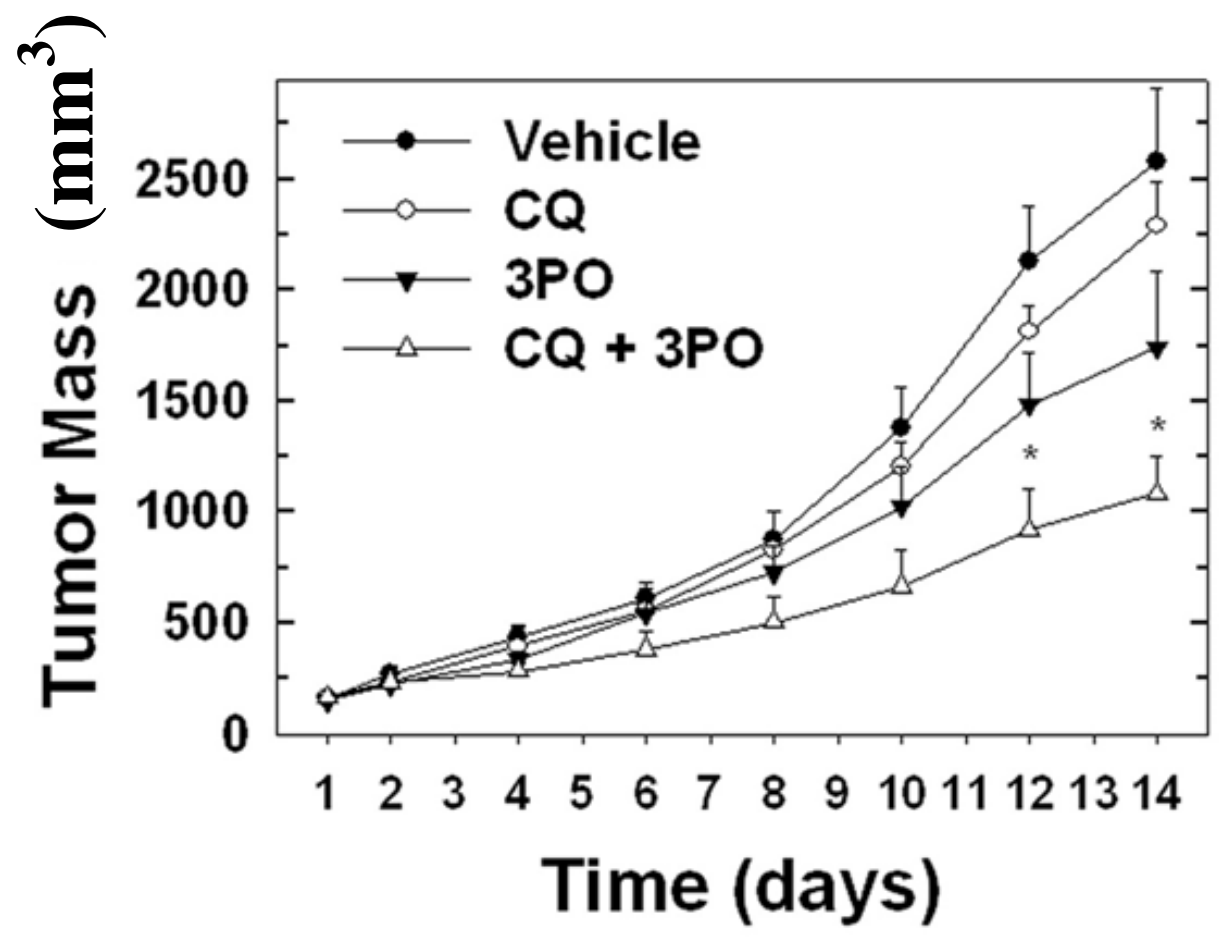


Figure 34: Immunohistochemistry of excised tumors from $3 \mathrm{PO}+$ chloroquine treated animals have increased cleaved-caspase 3 staining. Tumors were fixed, paraffin embedded and stained with an antibody directed against cleaved caspase-3 (CC3). The number of cells staining positive for CC3 was in a 200X field was counted and data is expressed as the mean \pm SD from three counts. $(p$ value $<0.05)$ 
Figure 34.

A.
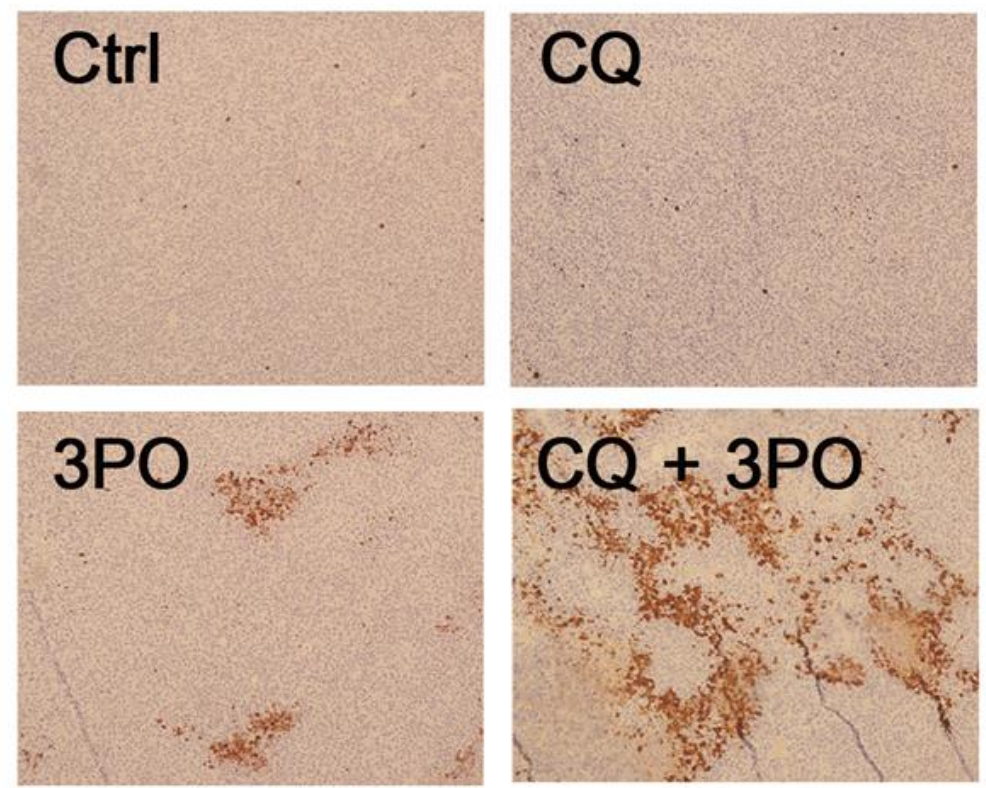

B.

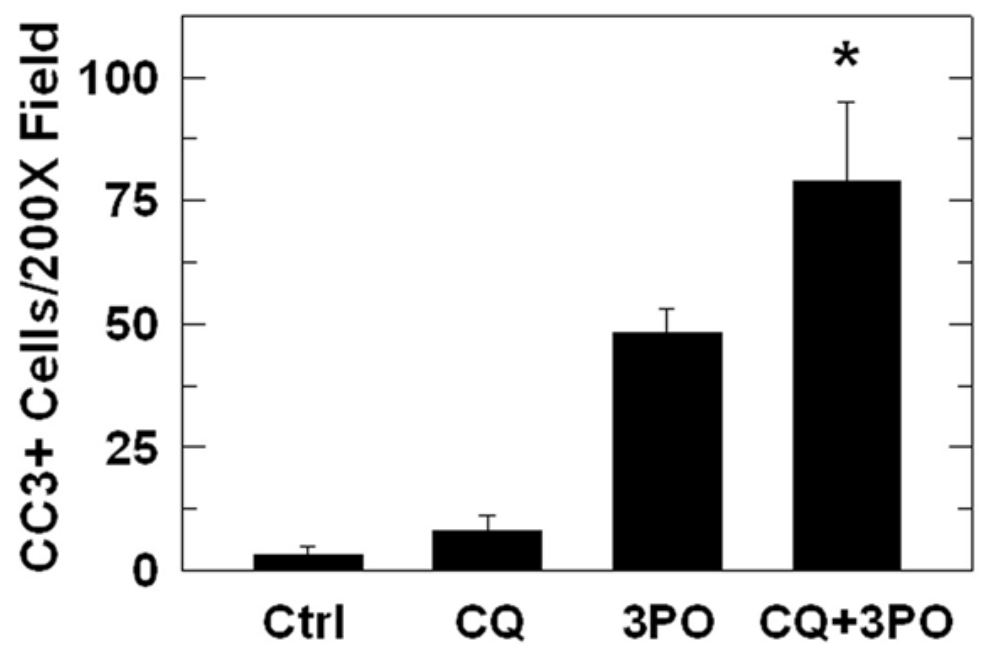




\section{Discussion}

The increasing identification of autophagy as a resistance mechanism utilized by tumor cells to avoid destruction led to postulation of improved antitumor effects by the combination of autophagy inhibitors with PFKFB3 inhibitors. We have shown that cell death following treatment with PFKFB3 inhibitor 3PO was increased when combined with autophagy inhibitors chloroquine, 3methyladeneine or Spautin-1. Additionally, chloroquine increased cell death when combined with other derivatives of the PFKFB3 inhibitor 3PO, PF-158 and PF-15, showing that the combination of chloroquine is not limited to only one PFKFB3 inhibitor. While these inhibitors, 3PO, PF-158 and PF-15, have been shown to inhibit PFKFB3 enzyme activity, these compounds most certainly have some off-target effects. For this reason, chloroquine was also tested in combination with PFKFB3 siRNA and enhanced cell death was noted. These observations support the role of autophagy as a resistance mechanism following the inhibition of PFKFB3 activity.

While in vitro research is absolutely essential to the understanding of cell function, data collected is limited by unforeseen effects that occur only in vivo. Animal studies are laborious, costly, and, even more importantly, result in the expenditure of numerous animal lives. Once sufficient data has been compiled from in vitro work, it is necessary and responsible to expand studies to an animal 
model system in order to increase its relevance. This is especially important when evaluating drugs or combinations of drugs due to unanticipated systemic effects that can occur.

Chloroquine has been safely used as an anti-malarial agent since the 1940's and has more recently been used as an anti-tumor agent. Based on previously reported animal tumor studies using chloroquine, a dose of $50 \mathrm{mg} / \mathrm{kg}$ was selected $[36,54,183,184]$. The dose of $3 P O$ used for this study $(0.07 \mathrm{mg} / \mathrm{g})$ was also selected based on a previously published animal tumor model [53]. The combination of 3PO and chloroquine resulted in significantly smaller tumors relative to either drug treatment alone. While there was a substantial difference in tumor size, it is important to note that $3 \mathrm{PO}$ treatment alone did not have the same anti-tumor effects that were seen in the previously published work [53], although the tumor model (Lewis lung carcinoma tumors in C57/BL6 mice) was the same. Additionally, although the model system was different, tumors from animals treated with chloroquine alone failed to show any difference in tumor size, contrasting with other published tumor studies $[183,184]$. While this may be due to the use of slower growing tumor models that are not as sensitive to chloroquine the LLC tumors grew very quickly, with the first tumors palpable merely three days after inoculation. Drug treatment was started when tumors reached between $150-200 \mathrm{~mm}^{3}$, calculated based on caliper measurements, which was less than one week after inoculation for most animals, in contrast to two to four weeks between inoculation and drug treatment in other models [183, 184]. In fact, in those studies it was not until more than three weeks after tumor 
inoculation that the average tumor mass in chloroquine-treated animals was smaller than the vehicle treated, which is longer than the length of the entire study presented here [184]. In this setting it is reasonable to speculate that the drugs may have had a difficult time competing with the accelerated growth of tumors. Future studies may benefit from using a slower growing model system so that treatment time can be extended.

Tumors that were removed from animals at the conclusion of the study were fixed and stained with a marker of cell death, cleaved-caspase 3. This marker was increased in tumors excised from animals treated with the combination of chloroquine and $3 \mathrm{PO}$ relative to those from animals treated with either drug alone. The smaller tumor size and increased CC3 staining supports the idea that autophagy is serving as a protective mechanism following PFKFB3 inhibition and that the efficacy of PFKFB3 inhibitors as anti-cancer agents may be improved using autophagy inhibitors such as chloroquine. 
Figure 35. Inhibition of glycolysis causes induction of autophagy for survival. Metabolic stress due to PFKFB3 knockdown results in AMPK activation and subsequent mTOR inhibition which relieves autophagy suppression allowing for increased cell survival. PFKFB3 inhibition using 3PO results in a much more rapid inhibition of glycolysis resulting in acute oxidative changes that lead to mTOR inhibition and autophagy induction. Adding chloroquine, an autophagy inhibitor, to either knockdown or to 3PO increases cell death. 
Figure 35.

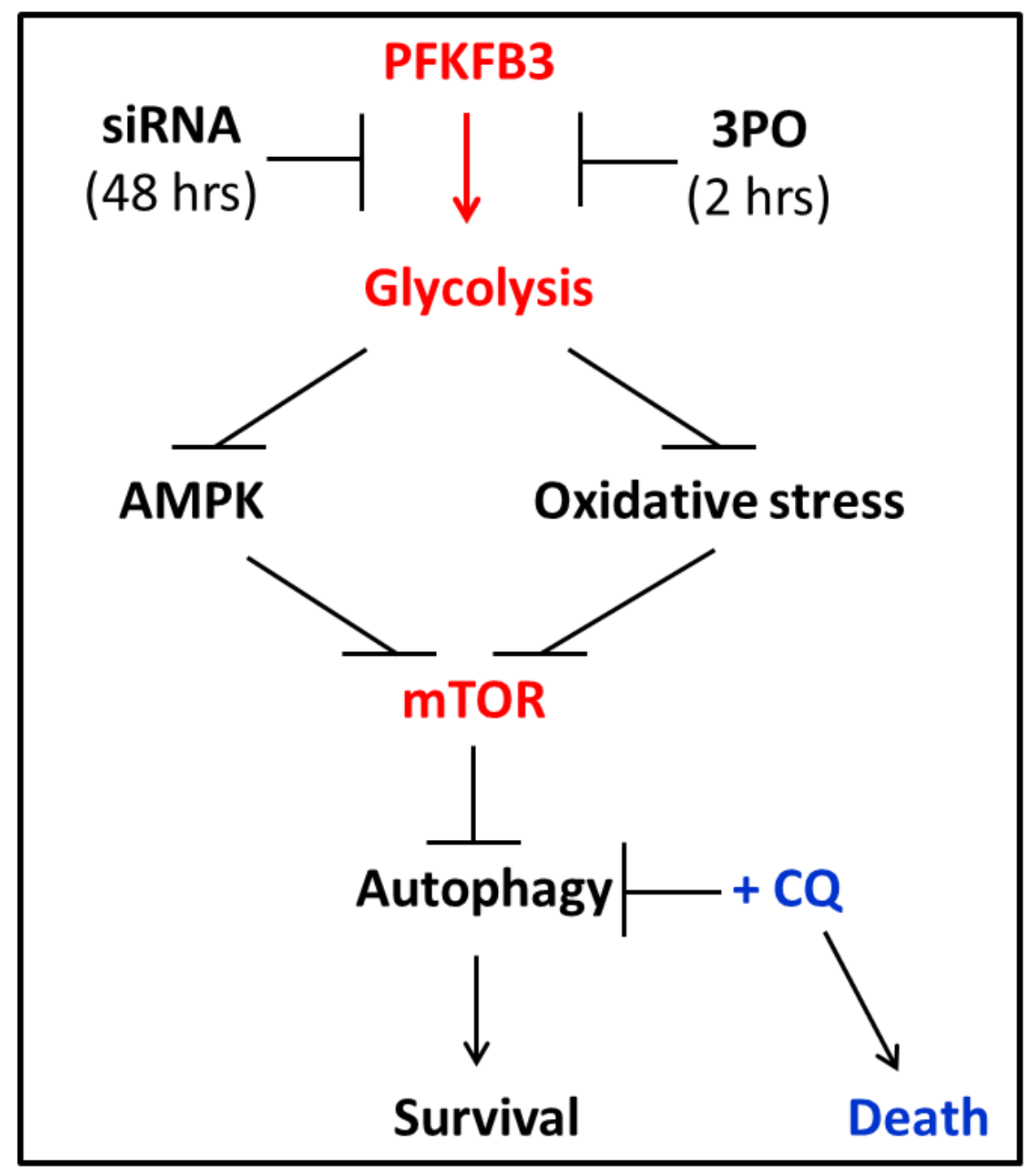




\section{CHAPTER V}

\section{SUMMARY}

Harnessing our knowledge gained from studying cancer cells over the past century in order to determine the traits that distinguish them from normal cells is paramount to developing cancer-specific therapies. Inhibition of 6phosphofructo-2-kinase/fructose-2,6-bisphosphatase 3 (PFKFB3) has proven to effectively and specifically target tumor cells in vitro and to decrease tumor burden in vivo [53]. Clinical trials using PFKFB3 inhibitors are forthcoming. However, like so many chemotherapeutics, it is not unexpected that resistance to these inhibitors could be encountered in the setting of human cancers. Resistance is often battled using combinations of drugs, which have now become standard, making single drug therapies quite rare. Elucidating the specific resistance mechanisms triggered by chemotherapeutic drugs allows for the selection of combinations that might work to combat such resistance with the hope of increasing efficacy. In this work we show that autophagy is induced by PFKFB3 inhibition and that this induction is likely serving as a resistance mechanism as supported by the observations of increased cell death in vitro and 
slowed tumor growth in vivo when combined with pharmacologic inhibitors of autophagy. It is likely that the use of drugs targeting PFKFB3 in human cancers will benefit from combinatorial therapies, such as that supported here with the autophagy inhibitor chloroquine. 


\section{FUTURE DIRECTIONS}

\section{EXPANSION OF MODELING SYSTEMS}

Like Galileo's heliocentric model of the universe, Warburg's theory regarding tumor cell metabolism, while still a major contribution to our understanding of tumor cell metabolism, may be flawed. He originally proposed that cancer cells utilized glycolysis due to damaged mitochondria preventing efficient oxidative respiration [2]. It is now thought that mitochondrial defects are somewhat rare and that most tumor cells not only have the capacity for but also utilize oxidative metabolism, albeit at reduced rates [185]. Still, it is clear that the increased uptake and utilization of glucose is a distinguishing attribute of tumor cells relative to normal cells, a feature that is taken advantage of for visualizing

tumors by PET scanning using 2-deoxy-2- $\left({ }^{18} \mathrm{~F}\right)$ fluoro-D-glucose positron emission tomography (FDG-PET) imaging.

To account for the increased uptake in glucose of tumors despite functional mitochondrial an alternative hypothesis, termed the "Reverse Warburg Effect" was proposed [186]. It postulates that the increased glycolytic flux in tumors is not due to the cancer cells themselves, but to stromal cells in the tumor microenvironment. In this model, oxidative stress resulting from cancer cell release of $\mathrm{H}_{2} \mathrm{O}_{2}$ stimulates stromal cells to undergo autophagy, and, in particular, 
selective mitochondrial autophagy, leaving them dependent on glycolysis for energy production [187, 188]. Thus, it is the stromal cancer-associated fibroblasts that are responsible for increased glucose uptake and utilization. These fibroblasts are then observed to release lactate and pyruvate as a result of increased glycolytic flux, which is taken up as a fuel for mitochondrial respiration in the cancer cells. In support of this theory, cancer cells co-cultured with fibroblasts were observed to have increased mitochondrial mass and decreased glycolytic flux relative to cancer cells cultured alone. Additionally, fibroblasts cocultured with cancer cells had decreased mitochondrial activity [189]. Whether it is due to stromal cells or to cancer cells or both, it is clear that tumors take up more glucose than normal tissues. It is reasonable to speculate that antiglycolytic drugs, such as PFKFB3 inhibitors, will be more effective as cytostatic or cytotoxic agents in cells with increased glycolytic potential. For this reason it would be important to return to the in vitro system and look at PFKFB3 inhibition in the setting of co-cultures of tumor cells and fibroblasts to determine if one cell type seems to be affected more than the other. In this same model system, other consequences of PFKFB3 inhibition, such as the induction of autophagy, could be measured to understand how cells within the tumor environment behave in.

Additionally, the work presented in this dissertation is limited by the model system used to evaluate PFKFB3 knockdown or inhibition. Future studies will also be conducted in multiple cell lines including a variety of tumor cells originating from different organs as well as in normal cells derived from primary cultures. It will also be important to study the combination of PFKFB3 inhibitors 
with more specific autophagy inhibitors. The use of chloroquine in this work was based on its action to inhibit autophagy and its long-standing safety profile in humans. As the autophagy field expands, it is likely that more inhibitors will be developed that have greater specificity for the autophagic process. This could mean that these agents are more tumor-specific and will have greater anti-tumor effects than chloroquine. As well as expanding the variety of drugs used to inhibit autophagy, it will be important to test newer derivatives of PFKFB3 inhibitors with fewer off-target effects and with lower toxicity ranges.

Another limitation of the work presented here is the singular in vivo model system. The animal study was performed using a mouse tumor cell line. More sophisticated, and also more expensive, xenograft modeling systems that utilize human cancer cells in an immunodeficient athymic mouse may more appropriately simulate the effect that PFKFB3 inhibitors will have on human cancers.

\section{UNDERSTANDING THE ROLE FOR GLUTAMINOLYSIS}

Other studies that could further our understanding of the role of PFKFB3 in cancer and the consequences of inhibition as a means to treat cancer should be focused on glutamine metabolism. Glutamine is another substrate, in addition to glucose, that is utilized to a greater extent in cancer cells relative to normal cells [190, 191]. Glucose deprivation is known to induce glutaminolysis which increases the synthesis of TCA intermediates, reduces oxidative stress by production of $\mathrm{NADPH}$, and also causes increased ammonia, a known inducer of 
autophagy [192-194]. Glutamine can also be used to make GSH. Glutaminase converts glutamine to glutamate which can be acted upon by glutathione cysteine ligase to be converted directly to GSH [195]. A gradual reduction in PFKFB3 using siRNA may result in the transition to utilization of glutamine as an alternative energy source. The subsequent metabolism of glutamine could be used to increase GSH, thus limiting reactive oxygen species. In fact, in data not shown, PFKFB3 knockdown was observed to result in less reactive oxygen species and in stabilization of the intracellular GSH pool. Conversely, the rapid decline in glucose uptake resulting from PFKFB3 inhibition using 3PO may lead to excessive reactive oxygen species that causes utilization and a decrease in the GSH pool, occurring before the cell has the time to utilize glutamine and counteract oxidative stress with increased GSH synthesis. Clearly it would be of great interest to study glutaminolysis in the setting of both PFKFB3 knockdown and PFKFB3 inhibition. As part of this endeavor, ${ }^{14} \mathrm{C}$-labeled glutamine might be used to measure glutamine uptake via scintillation counts in cells that treated with either PFKFB3 siRNA or with pharmacologic PFKFB3 inhibitors. Additionally, ${ }^{13} \mathrm{C}$-labeled glutamine could be used by nuclear magnetic resonance studies to understand how glutamine metabolism is altered in cells in which PFKFB3 is manipulated. Depending on the results of these studies, glutaminolytic modulators may be combined with PFKFB3 inhibitors to look for synergistic effects on tumor cell proliferation and viability. 


\section{EXPLOITING OXIDATIVE STRESS}

Another combination approach that may result in more effective cancer therapies is that of PFKFB3 inhibitors and drugs known to cause oxidative stress. The induction of ROS by PFKFB3 inhibition, and the additive effects on tumor cell death upon combination with chloroquine, a compound that, itself, has been shown to increase ROS, may mean that the combinatorial effects on cell death are due to a massive increase in ROS that tumor cells cannot battle [196]. There are a number of examples where utilization of drugs known to increase reactive oxygen species and oxidative stress have been successfully combined with chemotherapeutic drugs, a topic that has previously been reviewed [197]. Thus, it is possible that the combination of PFKFB3 inhibitors with these pro-oxidative agents will have even greater success than the combination with chloroquine. In fact, glucose-withdrawal induced oxidative stress has been observed to be greater in some tumor cells relative to normal cells which could indicate increased tumor specificity of this type of combination with PFKFB3 inhibitors [198]

In order to gain a greater understanding of how PFKFB3 manipulation affects oxidative stress, the source of stress must be elucidated. As previously stated, changes in glutamine metabolism may have a role in the production of GSH and, thus, the mitigation of damage that could be caused by ROS. Additionally, it will be important to measure the changes in mitochondrial mass, and function, by measuring membrane potential, oxygen consumption, and the 
production of mitochondrial-specific reactive oxygen species, after PFKFB3 manipulation.

\section{THE HUMAN PERSPECTIVE}

In vitro culture systems and in vivo animal modeling can only take us so far in the search for a cure to cancer. To treat human cancers we need to better understand their characteristics and functional requirements in their natural environment. Observing that some measure of metabolism or that expression of an activated protein in a pathway presumed to be important in cancer cells is changed after 6,12 , or 24 hours of treatment is not as meaningful as looking at what is happening in the majority of tumor cells at any point in time within the human body. Perhaps even more importantly, determining how tumors, as a whole, react to treatment with chemotherapeutics is critical to how we will move forward to treat tumors and to modify treatments based on a tumor's response to treatment. As part of this mission, it would be of great interest to examine samples taken from a variety of human tumors and measure PFKFB3 expression and markers of autophagy to look for correlations between glycolytic and autophagic activity. Additionally, once PFKFB3 inhibitors enter human cancer trials, tumor samples could be examined after treatment for assessment of autophagy markers. Correlations between the response of particular tumors, growth, shrinkage, or stabilization of tumor size with these autophagic markers could be used to make therapeutic modifications, for example, the addition of chloroquine or another inhibitor of autophagy. 
Work presented herein supports the protective role of autophagy in cancer cells following inhibition of PFKFB3, a key enzyme involved in the regulation of glycolysis. Treatment using PFKFB3 inhibitors and autophagy inhibitors, such as chloroquine, have shown promise as a combinatorial approach to tumor therapy. Still, as detailed above, a considerable amount of work needs to be accomplished to gain a better grasp on how to effectively target tumors by inhibiting PFKFB3. Hopefully the studies presented in this dissertation will serve as the groundwork for the development of novel and successful cancer anticancer strategies. 


\section{REFERENCES}

[1] Hanahan D, Weinberg RA. Hallmarks of cancer: the next generation. Cell 2011; 144:646-674.

[2] WARBURG O. On the origin of cancer cells. Science 1956; 123:309-314.

[3] Semenza GL. Oxygen-dependent regulation of mitochondrial respiration by hypoxia-inducible factor 1 . Biochem J 2007; 405:1-9.

[4] Vazquez A, Liu J, Zhou Y, Oltvai ZN. Catabolic efficiency of aerobic glycolysis: the Warburg effect revisited. BMC Syst Biol 2010; 4:58.

[5] Taylor RW, Turnbull DM. Mitochondrial DNA mutations in human disease. Nat Rev Genet 2005; 6:389-402.

[6] Carew JS, Huang P. Mitochondrial defects in cancer. Mol Cancer 2002; 1:9.

[7] Singh KK. Mitochondrial dysfunction is a common phenotype in aging and cancer. Ann N Y Acad Sci 2004; 1019:260-264.

[8] Brahimi-Horn C, Pouysségur J. When hypoxia signalling meets the ubiquitinproteasomal pathway, new targets for cancer therapy. Crit Rev Oncol Hematol 2005; 53:115-123.

[9] Godoy A, Ulloa V, Rodríguez $\mathrm{F}$ et al. Differential subcellular distribution of glucose transporters GLUT1-6 and GLUT9 in human cancer: ultrastructural localization of GLUT1 and GLUT5 in breast tumor tissues. J Cell Physiol 2006; 207:614-627.

[10] Cairns RA, Harris IS, Mak TW. Regulation of cancer cell metabolism. Nat Rev Cancer 2011; 11:85-95. 
[11] Gottlob K, Majewski N, Kennedy S et al. Inhibition of early apoptotic events by $A k t / P K B$ is dependent on the first committed step of glycolysis and mitochondrial hexokinase. Genes Dev 2001; 15:1406-1418.

[12] Rathmell JC, Fox CJ, Plas DR et al. Akt-directed glucose metabolism can prevent Bax conformation change and promote growth factor-independent survival. Mol Cell Biol 2003; 23:7315-7328.

[13] $\mathrm{Hu} \mathrm{Y,} \mathrm{Lu} \mathrm{W,} \mathrm{Chen} \mathrm{G}$ et al. K-ras(G12V) transformation leads to mitochondrial dysfunction and a metabolic switch from oxidative phosphorylation to glycolysis. Cell Res 2012; 22:399-412.

[14] Bustamante E, Pedersen PL. High aerobic glycolysis of rat hepatoma cells in culture: role of mitochondrial hexokinase. Proc Natl Acad Sci U S A 1977; 74:3735-3739.

[15] Coy JF, Dressler D, Wilde J, Schubert P. Mutations in the transketolase-like gene TKTL1: clinical implications for neurodegenerative diseases, diabetes and cancer. Clin Lab 2005; 51:257-273.

[16] Ramanathan A, Wang C, Schreiber SL. Perturbational profiling of a cell-line model of tumorigenesis by using metabolic measurements. Proc Natl Acad Sci U S A 2005; 102:5992-5997.

[17] Van Schaftingen E, Hue L, Hers HG. Fructose 2,6-bisphosphate, the probably structure of the glucose- and glucagon-sensitive stimulator of phosphofructokinase. Biochem J 1980; 192:897-901.

[18] Atsumi T, Nishio T, Niwa $\mathrm{H}$ et al. Expression of inducible 6-phosphofructo-2kinase/fructose-2,6-bisphosphatase/PFKFB3 isoforms in adipocytes and their potential role in glycolytic regulation. Diabetes 2005; 54:3349-3357.

[19] Bobarykina AY, Minchenko DO, Opentanova IL et al. Hypoxic regulation of PFKFB-3 and PFKFB-4 gene expression in gastric and pancreatic cancer cell lines and expression of PFKFB genes in gastric cancers. Acta Biochim Pol 2006; 53:789-799.

[20] Atsumi T, Chesney J, Metz C et al. High expression of inducible 6phosphofructo-2-kinase/fructose-2,6-bisphosphatase (iPFK-2; PFKFB3) in human cancers. Cancer Res 2002; 62:5881-5887. 
[21] Chen $C Y$, Shyu AB. AU-rich elements: characterization and importance in mRNA degradation. Trends Biochem Sci 1995; 20:465-470.

[22] Fukasawa $\mathrm{M}$, Tsuchiya $\mathrm{T}$, Takayama $\mathrm{E}$ et al. Identification and characterization of the hypoxia-responsive element of the human placental 6phosphofructo-2-kinase/fructose-2,6-bisphosphatase gene. J Biochem 2004; 136:273-277.

[23] Chesney J, Mitchell R, Benigni $\mathrm{F}$ et al. An inducible gene product for 6phosphofructo-2-kinase with an AU-rich instability element: role in tumor cell glycolysis and the Warburg effect. Proc Natl Acad Sci U S A 1999; 96:30473052.

[24] Okar DA, Manzano A, Navarro-Sabatè A et al. PFK-2/FBPase-2: maker and breaker of the essential biofactor fructose-2,6-bisphosphate. Trends Biochem Sci $2001 ; 26: 30-35$.

[25] Kitamura K, Uyeda K, Kangawa K, Matsuo H. Purification and characterization of rat skeletal muscle fructose-6-phosphate,2-kinase:fructose2,6-bisphosphatase. J Biol Chem 1989; 264:9799-9806.

[26] Calvo MN, Bartrons R, Castano E et al. PFKFB3 gene silencing decreases glycolysis, induces cell-cycle delay and inhibits anchorage-independent growth in HeLa cells. FEBS Lett 2006; 580:3308-3314.

[27] Xu RH, Pelicano $\mathrm{H}$, Zhou $\mathrm{Y}$ et al. Inhibition of glycolysis in cancer cells: a novel strategy to overcome drug resistance associated with mitochondrial respiratory defect and hypoxia. Cancer Res 2005; 65:613-621.

[28] Rockwell S, Schulz RJ. Failure of 5-thio-D-glucose to alter cell survival in irradiated or unirradiated EMT6 tumors. Radiat Res 1984; 100:527-535.

[29] Stein RC, Joseph AE, Matlin SA et al. A preliminary clinical study of gossypol in advanced human cancer. Cancer Chemother Pharmacol 1992; 30:480-482.

[30] Raez LE, Papadopoulos K, Ricart AD et al. A phase I dose-escalation trial of 2-deoxy-D-glucose alone or combined with docetaxel in patients with advanced solid tumors. Cancer Chemother Pharmacol 2013; 71:523-530. 
[31] Newell DR, Mansi J, Hardy J et al. The pharmacokinetics of oral lonidamine in breast and lung cancer patients. Semin Oncol 1991; 18:11-17.

[32] Stahl M, Schmoll E, Becker $\mathrm{H}$ et al. Lonidamine versus high-dose tamoxifen in progressive, advanced renal cell carcinoma: results of an ongoing randomized phase II study. Semin Oncol 1991; 18:33-37.

[33] BROWN J. Effects of 2-deoxyglucose on carbohydrate metablism: review of the literature and studies in the rat. Metabolism 1962; 11:1098-1112.

[34] Stein M, Lin H, Jeyamohan $\mathrm{C}$ et al. Targeting tumor metabolism with 2deoxyglucose in patients with castrate-resistant prostate cancer and advanced malignancies. Prostate 2010; 70:1388-1394.

[35] Goldberg SB, Supko JG, Neal JW et al. A phase I study of erlotinib and hydroxychloroquine in advanced non-small-cell lung cancer. $J$ Thorac Oncol 2012; 7:1602-1608.

[36] Sotelo J, Briceño E, López-González MA. Adding chloroquine to conventional treatment for glioblastoma multiforme: a randomized, double-blind, placebo-controlled trial. Ann Intern Med 2006; 144:337-343.

[37] Kyle RA, Seligman BR, Wallace HJ et al. Mutiple myeloma resistant to melphalan (NSC-8806) treated with cyclophosphamide (NSC-26271), prednisone (NSC-10023), and chloroquine (NSC-187208). Cancer Chemother Rep 1975; 59:557-562.

[38] Aki T, Yamaguchi K, Fujimiya T, Mizukami Y. Phosphoinositide 3-kinase accelerates autophagic cell death during glucose deprivation in the rat cardiomyocyte-derived cell line H9c2. Oncogene 2003; 22:8529-8535.

[39] Lock R, Roy S, Kenific CM et al. Autophagy facilitates glycolysis during Rasmediated oncogenic transformation. Mol Biol Cell 2011; 22:165-178.

[40] Mitchener JS, Shelburne JD, Bradford WD, Hawkins HK. Cellular autophagocytosis induced by deprivation of serum and amino acids in HeLa cells. Am J Pathol 1976; 83:485-492. 
[41] Lum JJ, Bauer DE, Kong $M$ et al. Growth factor regulation of autophagy and cell survival in the absence of apoptosis. Cell 2005; 120:237-248.

[42] Onodera J, Ohsumi Y. Autophagy is required for maintenance of amino acid levels and protein synthesis under nitrogen starvation. J Biol Chem 2005; 280:31582-31586.

[43] Singh R, Cuervo AM. Autophagy in the cellular energetic balance. Cell Metab 2011; 13:495-504.

[44] Rabinowitz JD, White E. Autophagy and metabolism. Science 2010; 330:1344-1348.

[45] DiPaola RS, Dvorzhinski D, Thalasila A et al. Therapeutic starvation and autophagy in prostate cancer: a new paradigm for targeting metabolism in cancer therapy. Prostate 2008; 68:1743-1752.

[46] Ben Sahra I, Laurent K, Giuliano S et al. Targeting cancer cell metabolism: the combination of metformin and 2-deoxyglucose induces p53-dependent apoptosis in prostate cancer cells. Cancer Res 2010; 70:2465-2475.

[47] Mai S, Muster B, Bereiter-Hahn J, Jendrach M. Autophagy proteins LC3B, ATG5 and ATG12 participate in quality control after mitochondrial damage and influence lifespan. Autophagy 2012; 8.

[48] Kim JH, Kim HY, Lee YK et al. Involvement of mitophagy in oncogenic KRas-induced transformation: overcoming a cellular energy deficit from glucose deficiency. Autophagy 2011; 7:1187-1198.

[49] Kanzawa T, Germano IM, Komata $\mathrm{T}$ et al. Role of autophagy in temozolomide-induced cytotoxicity for malignant glioma cells. Cell Death Differ 2004; 11:448-457.

[50] Katayama M, Kawaguchi T, Berger MS, Pieper RO. DNA damaging agentinduced autophagy produces a cytoprotective adenosine triphosphate surge in malignant glioma cells. Cell Death Differ 2007; 14:548-558. 
[51] Vazquez-Martin A, Oliveras-Ferraros C, Menendez JA. Autophagy facilitates the development of breast cancer resistance to the anti-HER2 monoclonal antibody trastuzumab. PLoS One 2009; 4:e6251.

[52] Carew JS, Nawrocki ST, Kahue CN et al. Targeting autophagy augments the anticancer activity of the histone deacetylase inhibitor SAHA to overcome BcrAbl-mediated drug resistance. Blood 2007; 110:313-322.

[53] Clem B, Telang S, Clem A et al. Small-molecule inhibition of 6phosphofructo-2-kinase activity suppresses glycolytic flux and tumor growth. Mol Cancer Ther 2008; 7:110-120.

[54] Lee KH, Hsu EC, Guh JH et al. Targeting energy metabolic and oncogenic signaling pathways in triple-negative breast cancer by a novel adenosine monophosphate-activated protein kinase (AMPK) activator. J Biol Chem 2011; 286:39247-39258.

[55] Sasaki K, Tsuno NH, Sunami E et al. Chloroquine potentiates the anticancer effect of 5-fluorouracil on colon cancer cells. BMC Cancer 2010; 10:370.

[56] Guo XL, Li D, Hu F et al. Targeting autophagy potentiates chemotherapyinduced apoptosis and proliferation inhibition in hepatocarcinoma cells. Cancer Lett 2012; 320:171-179.

[57] Wu Z, Chang PC, Yang JC et al. Autophagy Blockade Sensitizes Prostate Cancer Cells towards Src Family Kinase Inhibitors. Genes Cancer 2010; 1:40-49.

[58] Fan C, Wang W, Zhao B et al. Chloroquine inhibits cell growth and induces cell death in A549 lung cancer cells. Bioorg Med Chem 2006; 14:3218-3222.

[59] Gupta A, Roy S, Lazar AJ et al. Autophagy inhibition and antimalarials promote cell death in gastrointestinal stromal tumor (GIST). Proc Natl Acad Sci U S A 2010; 107:14333-14338.

[60] Dang CV. Links between metabolism and cancer. Genes Dev 2012; 26:877890. 
[61] Van Schaftingen E, Jett MF, Hue L, Hers HG. Control of liver 6phosphofructokinase by fructose 2,6-bisphosphate and other effectors. Proc Natl Acad Sci U S A 1981; 78:3483-3486.

[62] Casado M, Boscá L, Martín-Sanz P. Multiple forms of 6-phosphofructo-2kinase/fructose-2,6-bisphosphatase are expressed in perinatal rat liver. Am J Physiol 1996; 270:E244-250.

[63] Crepin KM, Darville MI, Hue L, Rousseau GG. Characterization of distinct mRNAs coding for putative isozymes of 6-phosphofructo-2-kinase/fructose-2,6bisphosphatase. Eur J Biochem 1989; 183:433-440.

[64] Kessler R, Eschrich K. Splice isoforms of ubiquitous 6-phosphofructo-2kinase/fructose-2,6-bisphosphatase in human brain. Brain Res Mol Brain Res 2001; 87:190-195.

[65] Sakakibara R, Kato M, Okamura $\mathrm{N}$ et al. Characterization of a human placental fructose-6-phosphate, 2-kinase/fructose-2,6-bisphosphatase. J Biochem 1997; 122:122-128.

[66] Shaw G, Kamen R. A conserved AU sequence from the 3' untranslated region of GM-CSF mRNA mediates selective mRNA degradation. Cell 1986; 46:659-667.

[67] Minchenko A, Leshchinsky I, Opentanova I et al. Hypoxia-inducible factor-1mediated expression of the 6-phosphofructo-2-kinase/fructose-2,6bisphosphatase-3 (PFKFB3) gene. Its possible role in the Warburg effect. J Biol Chem 2002; 277:6183-6187.

[68] Obach M, Navarro-Sabaté A, Caro J et al. 6-Phosphofructo-2-kinase (pfkfb3) gene promoter contains hypoxia-inducible factor-1 binding sites necessary for transactivation in response to hypoxia. J Biol Chem 2004; 279:53562-53570.

[69] Minchenko O, Opentanova I, Caro J. Hypoxic regulation of the 6phosphofructo-2-kinase/fructose-2,6-bisphosphatase gene family (PFKFB-1-4) expression in vivo. FEBS Lett 2003; 554:264-270.

[70] Riera L, Manzano A, Navarro-Sabaté A et al. Insulin induces PFKFB3 gene expression in HT29 human colon adenocarcinoma cells. Biochim Biophys Acta 2002; 1589:89-92. 
[71] Novellasdemunt L, Obach M, Millán-Ariño L et al. Progestins activate 6phosphofructo-2-kinase/fructose-2,6-bisphosphatase 3 (PFKFB3) in breast cancer cells. Biochem J 2012; 442:345-356.

[72] Telang S, Yalcin A, Clem AL et al. Ras transformation requires metabolic control by 6-phosphofructo-2-kinase. Oncogene 2006; 25:7225-7234.

[73] Manes NP, El-Maghrabi MR. The kinase activity of human brain 6phosphofructo-2-kinase/fructose-2,6-bisphosphatase is regulated via inhibition by phosphoenolpyruvate. Arch Biochem Biophys 2005; 438:125-136.

[74] Garcia-Cao I, Song MS, Hobbs RM et al. Systemic elevation of PTEN induces a tumor-suppressive metabolic state. Cell 2012; 149:49-62.

[75] Hamilton JA, Callaghan MJ, Sutherland RL, Watts CK. Identification of PRG1, a novel progestin-responsive gene with sequence homology to 6phosphofructo-2-kinase/fructose-2,6-bisphosphatase. Mol Endocrinol 1997; 11:490-502.

[76] Seo M, Kim JD, Neau D et al. Structure-based development of small molecule PFKFB3 inhibitors: a framework for potential cancer therapeutic agents targeting the Warburg effect. PLoS One 2011; 6:e24179.

[77] Fan QW, Cheng C, Hackett C et al. Akt and autophagy cooperate to promote survival of drug-resistant glioma. Sci Signal 2010; 3:ra81.

[78] Li J, Hou N, Faried A et al. Inhibition of autophagy augments 5-fluorouracil chemotherapy in human colon cancer in vitro and in vivo model. Eur $\mathrm{J}$ Cancer 2010; 46:1900-1909.

[79] Klionsky DJ. Autophagy revisited: a conversation with Christian de Duve. Autophagy 2008; 4:740-743.

[80] Huang J, Klionsky DJ. Autophagy and human disease. Cell Cycle 2007; 6:1837-1849.

[81] Tooze SA, Yoshimori T. The origin of the autophagosomal membrane. Nat Cell Biol 2010; 12:831-835. 
[82] Militello RD, Colombo MI. A membrane is born: origin of the autophagosomal compartment. Curr Mol Med 2011; 11:197-203.

[83] Mijaljica D, Prescott M, Devenish RJ. Endoplasmic reticulum and Golgi complex: Contributions to, and turnover by, autophagy. Traffic 2006; 7:15901595.

[84] Hamasaki M, Furuta N, Matsuda A et al. Autophagosomes form at ERmitochondria contact sites. Nature 2013; 495:389-393.

[85] Hailey DW, Rambold AS, Satpute-Krishnan P et al. Mitochondria supply membranes for autophagosome biogenesis during starvation. Cell 2010; 141:656-667.

[86] Girardi JP, Pereira L, Bakovic M. De novo synthesis of phospholipids is coupled with autophagosome formation. Med Hypotheses 2011; 77:1083-1087.

[87] Ravikumar B, Moreau K, Jahreiss L et al. Plasma membrane contributes to the formation of pre-autophagosomal structures. Nat Cell Biol 2010; 12:747-757.

[88] Kabeya Y, Mizushima N, Ueno T et al. LC3, a mammalian homologue of yeast Apg8p, is localized in autophagosome membranes after processing. EMBO J 2000; 19:5720-5728.

[89] Pankiv S, Clausen TH, Lamark T et al. p62/SQSTM1 binds directly to Atg8/LC3 to facilitate degradation of ubiquitinated protein aggregates by autophagy. J Biol Chem 2007; 282:24131-24145.

[90] Degenhardt K, Mathew R, Beaudoin B et al. Autophagy promotes tumor cell survival and restricts necrosis, inflammation, and tumorigenesis. Cancer Cell 2006; 10:51-64.

[91] Brown EJ, Albers MW, Shin TB et al. A mammalian protein targeted by G1arresting rapamycin-receptor complex. Nature 1994; 369:756-758.

[92] Kim DH, Sarbassov DD, Ali SM et al. mTOR interacts with raptor to form a nutrient-sensitive complex that signals to the cell growth machinery. Cell 2002; 110:163-175. 
[93] Holz MK, Ballif BA, Gygi SP, Blenis J. mTOR and S6K1 mediate assembly of the translation preinitiation complex through dynamic protein interchange and ordered phosphorylation events. Cell 2005; 123:569-580.

[94] Kim J, Kundu M, Viollet B, Guan KL. AMPK and mTOR regulate autophagy through direct phosphorylation of Ulk1. Nat Cell Biol 2011; 13:132-141.

[95] Hawley SA, Selbert MA, Goldstein EG et al. 5'-AMP activates the AMPactivated protein kinase cascade, and $\mathrm{Ca} 2+$ /calmodulin activates the calmodulindependent protein kinase I cascade, via three independent mechanisms. J Biol Chem 1995; 270:27186-27191.

[96] Suter M, Riek U, Tuerk R et al. Dissecting the role of 5'-AMP for allosteric stimulation, activation, and deactivation of AMP-activated protein kinase. J Biol Chem 2006; 281:32207-32216.

[97] Xiao B, Sanders MJ, Underwood E et al. Structure of mammalian AMPK and its regulation by ADP. Nature $2011 ; 472: 230-233$.

[98] Woods A, Johnstone SR, Dickerson K et al. LKB1 is the upstream kinase in the AMP-activated protein kinase cascade. Curr Biol 2003; 13:2004-2008.

[99] Lee JW, Park S, Takahashi Y, Wang HG. The association of AMPK with ULK1 regulates autophagy. PLoS One 2010; 5:e15394.

[100] Inoki K, Zhu T, Guan KL. TSC2 mediates cellular energy response to control cell growth and survival. Cell 2003; 115:577-590.

[101] Egan DF, Shackelford DB, Mihaylova MM et al. Phosphorylation of ULK1 (hATG1) by AMP-activated protein kinase connects energy sensing to mitophagy. Science 2011; 331:456-461.

[102] Kim J, Kundu M, Viollet B, Guan KL. AMPK and mTOR regulate autophagy through direct phosphorylation of Ulk1. Nat Cell Biol 2011; 13:132-141.

[103] Yin L, Kharbanda S, Kufe D. MUC1 oncoprotein promotes autophagy in a survival response to glucose deprivation. Int J Oncol 2009; 34:1691-1699. 
[104] Van Schaftingen E, Lederer B, Bartrons R, Hers HG. A kinetic study of pyrophosphate: fructose-6-phosphate phosphotransferase from potato tubers. Application to a microassay of fructose 2,6-bisphosphate. Eur J Biochem 1982; 129:191-195.

[105] Nixon RA, Wegiel J, Kumar A et al. Extensive involvement of autophagy in Alzheimer disease: an immuno-electron microscopy study. J Neuropathol Exp Neurol 2005; 64:113-122.

[106] Cordero MD, De Miguel M, Moreno Fernandez AM et al. Mitochondrial dysfunction and mitophagy activation in blood mononuclear cells of fibromyalgia patients: implications in the pathogenesis of the disease. Arthritis Res Ther 2010; 12:R17.

[107] Tasdemir E, Galluzzi L, Maiuri MC et al. Methods for assessing autophagy and autophagic cell death. Methods Mol Biol 2008; 445:29-76.

[108] Mizushima N, Yoshimori T. How to interpret LC3 immunoblotting. Autophagy 2007; 3:542-545.

[109] Yoshimori T, Yamamoto A, Moriyama $Y$ et al. Bafilomycin A1, a specific inhibitor of vacuolar-type $\mathrm{H}(+)$-ATPase, inhibits acidification and protein degradation in lysosomes of cultured cells. J Biol Chem 1991; 266:17707-17712.

[110] Bjørkøy G, Lamark T, Brech A et al. p62/SQSTM1 forms protein aggregates degraded by autophagy and has a protective effect on huntingtininduced cell death. J Cell Biol 2005; 171:603-614.

[111] Ylä-Anttila P, Vihinen H, Jokitalo E, Eskelinen EL. Monitoring autophagy by electron microscopy in Mammalian cells. Methods Enzymol 2009; 452:143-164.

[112] Bolster DR, Crozier SJ, Kimball SR, Jefferson LS. AMP-activated protein kinase suppresses protein synthesis in rat skeletal muscle through downregulated mammalian target of rapamycin (mTOR) signaling. J Biol Chem 2002; 277:23977-23980.

[113] Newsholme EA, Rolleston FS, Taylor K. Factors affecting the glucose 6phosphate inhibition of hexokinase from cerebral cortex tissue of the guinea pig. Biochem J 1968; 106:193-201. 
[114] Sutherland R, Freyer J, Mueller-Klieser W et al. Cellular growth and metabolic adaptations to nutrient stress environments in tumor microregions. Int J Radiat Oncol Biol Phys 1986; 12:611-615.

[115] Hoftiezer V, Berggren PO, Hellman B. Effects of glucose deprivation and altered $\mathrm{Ca} 2+$ concentrations on clonal insulin-producing cells (RINm5F). Biomed Biochim Acta 1985; 44:77-84.

[116] Zang Y, Yu LF, Nan FJ et al. AMP-activated protein kinase is involved in neural stem cell growth suppression and cell cycle arrest by 5 -aminoimidazole-4carboxamide-1-beta-D-ribofuranoside and glucose deprivation by downregulating phospho-retinoblastoma protein and cyclin D. J Biol Chem 2009; 284:6175-6184.

[117] Martinez-Outschoorn UE, Trimmer C, Lin Z et al. Autophagy in cancer associated fibroblasts promotes tumor cell survival: Role of hypoxia, HIF1 induction and NFKB activation in the tumor stromal microenvironment. Cell Cycle 2010; 9:3515-3533.

[118] Kim JH, Kim HY, Lee YK et al. Involvement of mitophagy in oncogenic KRas-induced transformation: overcoming a cellular energy deficit from glucose deficiency. Autophagy 2011; 7:1187-1198.

[119] Qu X, Zou Z, Sun $Q$ et al. Autophagy gene-dependent clearance of apoptotic cells during embryonic development. Cell 2007; 128:931-946.

[120] Kabeya Y, Mizushima N, Yamamoto A et al. LC3, GABARAP and GATE16 localize to autophagosomal membrane depending on form-II formation. J Cell Sci 2004; 117:2805-2812.

[121] Klionsky DJ, Elazar Z, Seglen PO, Rubinsztein DC. Does bafilomycin A1 block the fusion of autophagosomes with lysosomes? Autophagy 2008; 4:849950.

[122] Tanida I, Minematsu-Ikeguchi N, Ueno T, Kominami E. Lysosomal turnover, but not a cellular level, of endogenous LC3 is a marker for autophagy. Autophagy 2005; 1:84-91. 
[123] Vadlamudi RK, Shin J. Genomic structure and promoter analysis of the p62 gene encoding a non-proteasomal multiubiquitin chain binding protein. FEBS Lett 1998; 435:138-142.

[124] Moruno-Manchón JF, Pérez-Jiménez E, Knecht E. Glucose induces autophagy under starvation conditions by a p38 MAPK-dependent pathway. Biochem J 2013; 449:497-506.

[125] Marambio P, Toro B, Sanhueza C et al. Glucose deprivation causes oxidative stress and stimulates aggresome formation and autophagy in cultured cardiac myocytes. Biochim Biophys Acta 2010; 1802:509-518.

[126] Xi H, Kurtoglu M, Liu $\mathrm{H}$ et al. 2-Deoxy-D-glucose activates autophagy via endoplasmic reticulum stress rather than ATP depletion. Cancer Chemother Pharmacol 2011; 67:899-910.

[127] Hariharan N, Maejima $Y$, Nakae J et al. Deacetylation of FoxO by Sirt1 Plays an Essential Role in Mediating Starvation-Induced Autophagy in Cardiac Myocytes. Circ Res 2010; 107:1470-1482.

[128] Kalender A, Selvaraj A, Kim SY et al. Metformin, independent of AMPK, inhibits mTORC1 in a rag GTPase-dependent manner. Cell Metab 2010; 11:390401.

[129] Liu Y, Song XD, Liu W et al. Glucose deprivation induces mitochondrial dysfunction and oxidative stress in PC12 cell line. J Cell Mol Med 2003; 7:49-56.

[130] Scherz-Shouval R, Elazar Z. Regulation of autophagy by ROS: physiology and pathology. Trends Biochem Sci 2011; 36:30-38.

[131] Scherz-Shouval R, Shvets E, Fass E et al. Reactive oxygen species are essential for autophagy and specifically regulate the activity of Atg4. EMBO J 2007; 26:1749-1760.

[132] Zhang H, Bosch-Marce M, Shimoda LA et al. Mitochondrial autophagy is an HIF-1-dependent adaptive metabolic response to hypoxia. J Biol Chem 2008; 283:10892-10903. 
[133] Nakatogawa H, Ishii J, Asai E, Ohsumi Y. Atg4 recycles inappropriately lipidated Atg8 to promote autophagosome biogenesis. Autophagy 2012; 8:177186.

[134] Chen Y, Azad MB, Gibson SB. Superoxide is the major reactive oxygen species regulating autophagy. Cell Death Differ 2009; 16:1040-1052.

[135] Frank M, Duvezin-Caubet S, Koob S et al. Mitophagy is triggered by mild oxidative stress in a mitochondrial fission dependent manner. Biochim Biophys Acta 2012; 1823:2297-2310.

[136] Guo H, Chen Y, Liao L, Wu W. Resveratrol Protects HUVECs from Oxidized-LDL Induced Oxidative Damage by Autophagy Upregulation via the AMPK/SIRT1 Pathway. Cardiovasc Drugs Ther 2013; 27:189-198.

[137] Xiong Y, Contento AL, Nguyen PQ, Bassham DC. Degradation of oxidized proteins by autophagy during oxidative stress in Arabidopsis. Plant Physiol 2007; 143:291-299.

[138] Dutta D, Xu J, Kim JS et al. Upregulated autophagy protects cardiomyocytes from oxidative stress-induced toxicity. Autophagy 2013; 9:328344.

[139] Zhang $\mathrm{H}$, Kong $\mathrm{X}$, Kang $\mathrm{J}$ et al. Oxidative stress induces parallel autophagy and mitochondria dysfunction in human glioma U251 cells. Toxicol Sci 2009; 110:376-388.

[140] Wang Q, Liang B, Shirwany NA, Zou MH. 2-Deoxy-D-glucose treatment of endothelial cells induces autophagy by reactive oxygen species-mediated activation of the AMP-activated protein kinase. PLoS One 2011; 6:e17234.

[141] Byun YJ, Kim SK, Kim YM et al. Hydrogen peroxide induces autophagic cell death in C6 glioma cells via BNIP3-mediated suppression of the mTOR pathway. Neurosci Lett 2009; 461:131-135.

[142] Vucicevic L, Misirkic M, Janjetovic $\mathrm{K}$ et al. Compound $\mathrm{C}$ induces protective autophagy in cancer cells through AMPK inhibition-independent blockade of Akt/mTOR pathway. Autophagy 2011; 7:40-50. 
[143] Essick EE, Sam F. Oxidative stress and autophagy in cardiac disease, neurological disorders, aging and cancer. Oxid Med Cell Longev 2010; 3:168177.

[144] McCubrey JA, Franklin RA. Reactive oxygen intermediates and signaling through kinase pathways. Antioxid Redox Signal 2006; 8:1745-1748.

[145] Rada B, Hably C, Meczner A et al. Role of Nox2 in elimination of microorganisms. Semin Immunopathol 2008; 30:237-253.

[146] Sareila O, Kelkka T, Pizzolla A et al. NOX2 complex-derived ROS as immune regulators. Antioxid Redox Signal 2011; 15:2197-2208.

[147] Hamanaka RB, Chandel NS. Mitochondrial reactive oxygen species regulate cellular signaling and dictate biological outcomes. Trends Biochem Sci 2010; 35:505-513.

[148] Filomeni G, Aquilano K, Civitareale P et al. Activation of c-Jun-N-terminal kinase is required for apoptosis triggered by glutathione disulfide in neuroblastoma cells. Free Radic Biol Med 2005; 39:345-354.

[149] Minich T, Riemer J, Schulz JB et al. The multidrug resistance protein 1 (Mrp1), but not Mrp5, mediates export of glutathione and glutathione disulfide from brain astrocytes. J Neurochem 2006; 97:373-384.

[150] Han YH, Kim SH, Kim SZ, Park WH. Apoptosis in arsenic trioxide-treated Calu-6 lung cells is correlated with the depletion of GSH levels rather than the changes of ROS levels. J Cell Biochem 2008; 104:862-878.

[151] Xie $Y$, Kole S, Precht $P$ et al. S-glutathionylation impairs signal transducer and activator of transcription 3 activation and signaling. Endocrinology 2009; 150:1122-1131.

[152] Huang Z, Pinto JT, Deng H, Richie JP. Inhibition of caspase-3 activity and activation by protein glutathionylation. Biochem Pharmacol 2008; 75:2234-2244.

[153] Liu L, Wise DR, Diehl JA, Simon MC. Hypoxic reactive oxygen species regulate the integrated stress response and cell survival. J Biol Chem 2008; 283:31153-31162. 
[154] Alexander A, Cai SL, Kim J et al. ATM signals to TSC2 in the cytoplasm to regulate mTORC1 in response to ROS. Proc Natl Acad Sci U S A 2010; 107:4153-4158.

[155] Bhogal RH, Weston CJ, Curbishley SM et al. Autophagy: a cyto-protective mechanism which prevents primary human hepatocyte apoptosis during oxidative stress. Autophagy 2012; 8:545-558.

[156] Liang $\mathrm{XH}$, Jackson S, Seaman $\mathrm{M}$ et al. Induction of autophagy and inhibition of tumorigenesis by beclin 1. Nature 1999; 402:672-676.

[157] Xie HJ, Noh JH, Kim JK et al. HDAC1 inactivation induces mitotic defect and caspase-independent autophagic cell death in liver cancer. PLoS One 2012; 7:e34265.

[158] Degtyarev M, De Mazière A, Orr C et al. Akt inhibition promotes autophagy and sensitizes PTEN-null tumors to lysosomotropic agents. J Cell Biol 2008; 183:101-116.

[159] Furuta S, Hidaka E, Ogata A et al. Ras is involved in the negative control of autophagy through the class I PI3-kinase. Oncogene 2004; 23:3898-3904.

[160] Arico S, Petiot A, Bauvy C et al. The tumor suppressor PTEN positively regulates macroautophagy by inhibiting the phosphatidylinositol 3-kinase/protein kinase B pathway. J Biol Chem 2001; 276:35243-35246.

[161] Crighton D, Wilkinson S, Ryan KM. DRAM links autophagy to p53 and programmed cell death. Autophagy 2007; 3:72-74.

[162] Tasdemir E, Maiuri MC, Galluzzi L et al. Regulation of autophagy by cytoplasmic p53. Nat Cell Biol 2008; 10:676-687.

[163] Fung C, Lock R, Gao S et al. Induction of autophagy during extracellular matrix detachment promotes cell survival. Mol Biol Cell 2008; 19:797-806.

[164] Lu Z, Luo RZ, Lu Y et al. The tumor suppressor gene ARHI regulates autophagy and tumor dormancy in human ovarian cancer cells. J Clin Invest 2008; 118:3917-3929. 
[165] Sosa MS, Bragado P, Debnath J, Aguirre-Ghiso JA. Regulation of tumor cell dormancy by tissue microenvironments and autophagy. Adv Exp Med Biol 2013; 734:73-89.

[166] Kanematsu S, Uehara N, Miki $\mathrm{H}$ et al. Autophagy inhibition enhances sulforaphane-induced apoptosis in human breast cancer cells. Anticancer Res 2010; 30:3381-3390.

[167] Amaravadi RK, Yu D, Lum JJ et al. Autophagy inhibition enhances therapyinduced apoptosis in a Myc-induced model of lymphoma. J Clin Invest 2007; 117:326-336.

[168] Ding WX, Ni HM, Gao W et al. Oncogenic transformation confers a selective susceptibility to the combined suppression of the proteasome and autophagy. Mol Cancer Ther 2009; 8:2036-2045.

[169] Burger H, van Tol H, Boersma AW et al. Imatinib mesylate (STI571) is a substrate for the breast cancer resistance protein (BCRP)/ABCG2 drug pump. Blood 2004; 104:2940-2942.

[170] Pommier Y, Sordet O, Antony S et al. Apoptosis defects and chemotherapy resistance: molecular interaction maps and networks. Oncogene 2004; 23:29342949.

[171] Andersag $\mathrm{H}$, Breitner $\mathrm{S}$, Jung $\mathrm{H}$. Quinoline compound and process of making the same. German Pat. 683 692. Chem. Abstr. 36, 4973. In.

[172] Woodward RB, Doering WE. The total synthesis of quinine. In: J. Am. Chem. Soc.: 1944. pp. 849-849.

[173] Kunze H, Hesse B, Bohn E. Effects of antimalarial drugs on several rat-liver lysosomal enzymes involved in phosphatidylethanolamine catabolism. Biochim Biophys Acta 1982; 713:112-117.

[174] Nosál R, Jancinová V. Cationic amphiphilic drugs and platelet phospholipase A(2) (cPLA(2)). Thromb Res 2002; 105:339-345.

[175] O'Neill PM, Bray PG, Hawley SR et al. 4-Aminoquinolines--past, present, and future: a chemical perspective. Pharmacol Ther 1998; 77:29-58. 
[176] Kremer JM. Rational use of new and existing disease-modifying agents in rheumatoid arthritis. Ann Intern Med 2001; 134:695-706.

[177] Romanelli F, Smith KM, Hoven AD. Chloroquine and hydroxychloroquine as inhibitors of human immunodeficiency virus (HIV-1) activity. Curr Pharm Des 2004; 10:2643-2648.

[178] Geser A, Brubaker G, Draper CC. Effect of a malaria suppression program on the incidence of African Burkitt's lymphoma. Am J Epidemiol 1989; 129:740752.

[179] Dang CV. Antimalarial therapy prevents Myc-induced lymphoma. J Clin Invest 2008; 118:15-17.

[180] Krmpot AJ, Janjetovic KD, Misirkic MS et al. Protective effect of autophagy in laser-induced glioma cell death in vitro. Lasers Surg Med 2010; 42:338-347.

[181] Solomon VR, Lee H. Chloroquine and its analogs: a new promise of an old drug for effective and safe cancer therapies. Eur J Pharmacol 2009; 625:220233.

[182] Yang S, Wang X, Contino G et al. Pancreatic cancers require autophagy for tumor growth. Genes Dev 2011; 25:717-729.

[183] Zheng $Y$, Zhao $Y L$, Deng $X$ et al. Chloroquine inhibits colon cancer cell growth in vitro and tumor growth in vivo via induction of apoptosis. Cancer Invest 2009; 27:286-292.

[184] Jiang PD, Zhao YL, Deng XQ et al. Antitumor and antimetastatic activities of chloroquine diphosphate in a murine model of breast cancer. Biomed Pharmacother 2010; 64:609-614.

[185] Hamanaka RB, Chandel NS. Cell biology. Warburg effect and redox balance. Science 2011; 334:1219-1220.

[186] Pavlides S, Whitaker-Menezes D, Castello-Cros R et al. The reverse Warburg effect: aerobic glycolysis in cancer associated fibroblasts and the tumor stroma. Cell Cycle 2009; 8:3984-4001. 
[187] Martinez-Outschoorn UE, Pavlides S, Whitaker-Menezes D et al. Tumor cells induce the cancer associated fibroblast phenotype via caveolin-1 degradation: implications for breast cancer and DCIS therapy with autophagy inhibitors. Cell Cycle 2010; 9:2423-2433.

[188] Martinez-Outschoorn UE, Pavlides S, Howell A et al. Stromal-epithelial metabolic coupling in cancer: integrating autophagy and metabolism in the tumor microenvironment. Int J Biochem Cell Biol 2011; 43:1045-1051.

[189] Martinez-Outschoorn UE, Lin Z, Trimmer C et al. Cancer cells metabolically "fertilize" the tumor microenvironment with hydrogen peroxide, driving the Warburg effect: implications for PET imaging of human tumors. Cell Cycle 2011; 10:2504-2520.

[190] Bode BP, Souba WW. Modulation of cellular proliferation alters glutamine transport and metabolism in human hepatoma cells. Ann Surg 1994; 220:411422; discussion 422-414.

[191] Kovacević Z, Morris HP. The role of glutamine in the oxidative metabolism of malignant cells. Cancer Res 1972; 32:326-333.

[192] Eng CH, Abraham RT. Glutaminolysis yields a metabolic by-product that stimulates autophagy. Autophagy 2010; 6:968-970.

[193] Le A, Lane AN, Hamaker $M$ et al. Glucose-independent glutamine metabolism via TCA cycling for proliferation and survival in B cells. Cell Metab 2012; 15:110-121.

[194] Mariño G, Kroemer G. Ammonia: a diffusible factor released by proliferating cells that induces autophagy. Sci Signal 2010; 3:pe19.

[195] Meister A. On the enzymology of amino acid transport. Science 1973; 180:33-39.

[196] Park J, Choi K, Jeong $\mathrm{E}$ et al. Reactive oxygen species mediate chloroquine-induced expression of chemokines by human astroglial cells. Glia 2004; 47:9-20. 
[197] Trachootham D, Alexandre J, Huang P. Targeting cancer cells by ROSmediated mechanisms: a radical therapeutic approach? Nat Rev Drug Discov 2009; 8:579-591.

[198] Jelluma N, Yang X, Stokoe D et al. Glucose withdrawal induces oxidative stress followed by apoptosis in glioblastoma cells but not in normal human astrocytes. Mol Cancer Res 2006; 4:319-330. 


\section{APPENDIX}




\title{
DNA Polymerase Eta Participates in the Mutagenic Bypass of Adducts Induced by Benzo[a]pyrene Diol Epoxide in Mammalian Cells
}

\author{
Alden C. Klarer ${ }^{1,29}$, L. Jay Stallons ${ }^{4 * 9 \times a}$, Tom J. Burke ${ }^{3}$, Robert L. Skaggs ${ }^{4 \times b}$, W. Glenn McGregor ${ }^{2,4}$ \\ 1 Department of Biochemistry and Molecular Biology, University of Louisville, Louisville, Kentucky, United States of America, 2 James Graham Brown Cancer Center, \\ University of Louisville, Louisville, Kentucky, United States of America, 3 Department of Medicine, Division of Gastroenterology, Hepatology and Nutrition, University of \\ Louisville, Louisville, Kentucky, United States of America, 4Department of Pharmacology and Toxicology, University of Louisville, Louisville, Kentucky, United States of \\ America
}

\begin{abstract}
Y-family DNA-polymerases have larger active sites that can accommodate bulky DNA adducts allowing them to bypass these lesions during replication. One member, polymerase eta (pol eta), is specialized for the bypass of UV-induced thymidine-thymidine dimers, correctly inserting two adenines. Loss of pol eta function is the molecular basis for xeroderma pigmentosum (XP) variant where the accumulation of mutations results in a dramatic increase in UV-induced skin cancers. Less is known about the role of pol eta in the bypass of other DNA adducts. A commonly encountered DNA adduct is that caused by benzo[a]pyrene diol epoxide (BPDE), the ultimate carcinogenic metabolite of the environmental chemical benzo[a]pyrene. Here, treatment of pol eta-deficient fibroblasts from humans and mice with BPDE resulted in a significant decrease in Hprt gene mutations. These studies in mammalian cells support a number of in vitro reports that purified pol eta has error-prone activity on plasmids with site-directed BPDE adducts. Sequencing the Hprt gene from this work shows that the majority of mutations are $\mathrm{G}>\mathrm{T}$ transversions. These data suggest that pol eta has error-prone activity when bypassing BPDE-adducts. Understanding the basis of environmental carcinogen-derived mutations may enable prevention strategies to reduce such mutations with the intent to reduce the number of environmentally relevant cancers.

Citation: Klarer AC, Stallons U, Burke TJ, Skaggs RL, McGregor WG (2012) DNA Polymerase Eta Participates in the Mutagenic Bypass of Adducts Induced by Benzo[a]pyrene Diol Epoxide in Mammalian Cells. PLoS ONE 7(6): e39596. doi:10.1371/journal.pone.0039596

Editor: John R. Battista, Louisiana State University and A \& M College, United States of America

Received January 25, 2012; Accepted May 28, 2012; Published June 20, 2012

Copyright: @ 2012 Klarer et al. This is an open-access article distributed under the terms of the Creative Commons Attribution License, which permits unrestricted use, distribution, and reproduction in any medium, provided the original author and source are credited.

Funding: This work was partially supported by USPHS Grant T32-ES011564, by a predoctoral fellowship to US, F30-ES017730 to ACK, and by R01-CA112197 and R03-CA112664 to WGM. The funders had no role in study design, data collection and analysis, decision to publish, or preparation of the manuscript.

Competing Interests: The authors have declared that no competing interests exist.

*E-mail: stallons@musc.edu

These authors contributed equally to this work.

aa Current address: Department of Pharmaceutical and Biomedical Sciences, Medical University of South Carolina, Charleston, South Carolina, United States of America

ab Current address: University of Kentucky College of Medicine, Lexington, Kentucky, United States of America
\end{abstract}

\section{Introduction}

Environmental mutagens such as the ultraviolet (UV) component of sunlight and chemical mutagens in food and cigarette smoke are well-established human carcinogens. One such compound is benzo[a]pyrene $(\mathrm{B}[\mathrm{a}] \mathrm{P})$, which is a polycyclic aromatic hydrocarbon present in cigarette smoke, diesel exhaust and wellcooked meat. The carcinogenic effects of $\mathrm{B}[\mathrm{a}] \mathrm{P}$ are largely due to its metabolism to the highly reactive product benzo[a]pyrene diol epoxide (BPDE), which principally binds to the exocyclic amine of guanine [1]. Nucleotide excision repair recognizes and removes adducts induced by BPDE using transcription-coupled and global genomic repair mechanisms $[2,3]$. However, if a cell enters Sphase with persistent damage, helix-distorting lesions halt the progression of the replication apparatus. Data indicate that specialized DNA polymerases that have relaxed base-pairing requirements may facilitate bypass of bulky DNA adducts in a process called translesion DNA synthesis (TLS), but with potentially mutagenic consequences. A subset of these proteins has been classified as Y-family polymerases based on structural similarities [4].

There are four members of the Y-family: REVl, pol eta, pol iota, and pol kappa. The role of these proteins in human disease is best characterized for pol eta. Loss of pol eta activity is the molecular defect underlying the XP variant syndrome $[5,6]$. These patients have a very high incidence of sunlight-induced skin cancer but are nucleotide-excision repair proficient unlike the classic XP complementation groups A G [7]. Cells from these patients are extremely hypermutable after exposure to UV due to the deficiency of pol eta which normally bypasses the most common UV-induced photoproducts, thymine-thymine cyclobutane pyrimidine dimers, in an error-free manner $[8,9]$. In the absence of pol eta, the highly error-prone pol iota assumes this bypass function resulting in the accumulation of UV-induced mutations and an increased susceptibility to skin cancer [ $\left[\begin{array}{ll}10 & 12\end{array}\right]$.

The role of the Y-family proteins in the bypass of BPDEinduced adducts is considerably less clear. Pol kappa has been shown to participate in error-free bypass of lesions induced by 
BPDE in vitro using purified mouse and human enzymes [ $\left.\begin{array}{ll}13 & 15\end{array}\right]$. Similar conclusions were reported using mouse cells lacking pol kappa activity $[15,16]$. While there are some data concerning the role of pol eta in TLS of BPDE-induced DNA adducts, the data are somewhat contradictory. Purified pol eta has been shown to be capable of performing error-prone bypass of BPDE-adducted plasmids in vitro $[13,17,18]$ and in mammalian cells using shuttle vectors to measure TLS efficiency and fidelity [19]. However, measurements of mutations at the endogenous hypoxanthineguanine phosphoribosyltransferase (HPRT) locus in pol etadeficient human cells treated with BPDE suggest that pol eta is error-free in bypassing BPDE-induced DNA adducts [20].

The $\mathrm{B}$ family member pol zeta is also implicated in the bypass of BPDE lesions. Purified pol zeta has alternatively been shown to have error-free [21] and error-prone [22,23] bypass activity on BPDE-adducted DNA templates in vitro. In addition, pol zeta deficiency has been shown to affect TLS of BPDE lesions in mammalian cells $[24,25]$.

Here, BPDE treatment of both cells derived from pol etaknockout mice and pol eta-deficient cells from an XP variant patient resulted in lower induced mutant frequencies. Our results suggest that pol eta is involved in error-prone bypass of BPDEinduced lesions and may be partially responsible for the mutagenic effects of this carcinogen. Understanding the mechanisms by which environmental chemicals induce mutations in DNA increases our understanding of how cancers can be linked to the environment and will increase our ability to develop strategies to prevent such cancers, for example through tissue-specific selective inhibition of one or more of these enzymes.

\section{Results}

Cytotoxicity from BPDE exposure is independent of pol eta status

The survival of mouse primary fibroblasts after treatment with $150 \mathrm{nM}$ BPDE was assessed using clonogenic assays. Wild-type and pol eta knockout cells exhibited similar levels of cytotoxicity when exposed to $150 \mathrm{nM}$ BPDE $(41 \pm 5 \%$ and $37 \pm 8 \%$ survival, respectively, $p=0.67$ ) (Figure 1). Human cells with wild-type pol eta and XP variant cells without functional pol eta exhibited similar sensitivities to $150 \mathrm{nM}$ BPDE $(46 \pm 9 \%$ and $36 \pm 10 \%$, respectively, $p=0.51$ ) (Figure 1). Mutagenic responses could

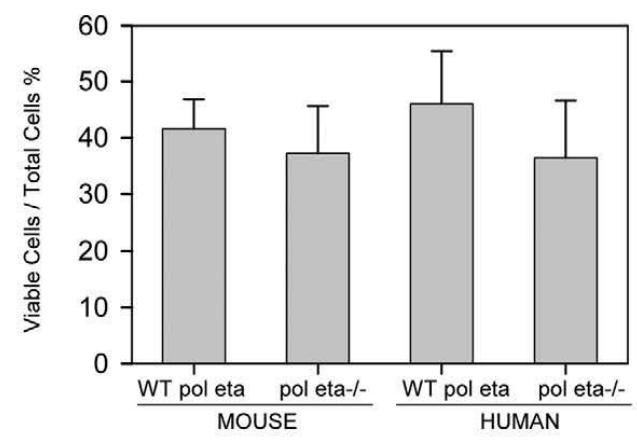

Figure 1. Polymerase eta does not affect cell survival after 15OnM BPDE treatment. Viable cell calculations were made based on the number of clones present relative to the number of cells plated. This value was adjusted based on the number of clones observed in vehicle-only treated cells and expressed as average \pm SEM. doi:10.1371/journal.pone.0039596.g001 therefore be assessed in comparable numbers of surviving cells after treatment

Reduced BPDE-induced mutant frequency was observed in the absence of pol eta

Fibroblasts deficient for pol eta were treated with $150 \mathrm{nM}$ BPDE and mutant frequency was assessed by the formation of 6 thioguanine-(TG) resistant clones. The induced mutant frequency of wild type mouse fibroblasts in response to $150 \mathrm{nM}$ BPDE was $213 \pm 48$ mutants per $1 \times 10^{6}$ cells. Relative to wild-type, the BPDE-induced mutant frequency in pol eta null mouse fibroblasts was significantly decreased, $34 \pm 10$ mutants per $1 \times 10^{6}$ cells $(p<0.05)$ (Figure 2).

Similarly, wild type human fibroblasts had a much higher induced mutant frequency relative to $\mathrm{XP}$ variant fibroblasts containing a non-functional pol eta. Normal human fibroblasts had an induced mutant frequency of $337 \pm 56$ mutants per $1 \times 10^{6}$ cells while the induced mutant frequency in XP variant fibroblasts was $96 \pm 24$ mutants per $1 \times 10^{6}$ cells $(p<0.05)$ (Figure 2). Both human and mouse cells had low background mutation frequency which was not affected by the loss of pol eta (data not shown).

\section{Sequencing of Hprt mutant clones}

After two weeks of growth in selective medium, macroscopic colonies of murine fibroblasts were isolated and lysed for RT-PCR amplification of Hprt cDNA and subsequent sequence analysis. Overall, the majority of the mutations in these mutant clones were guanine to thymine transversions which accounted for $50 \%$ and $70 \%$ of the mutations in wild-type and pol eta-null fibroblasts, respectively (Table 1).

\section{Discussion}

The somatic mutation hypothesis of cancer asserts that changes arising from mutations in the genome can result in cells with a growth advantage and thus tumorigenic potential. While it is currently estimated that the spontaneous mutation rate in human cells is around $12 \times 10^{-8}$ per nucleotide per generation [26], the overwhelming evidence for environmental carcinogen-induced cancers is attributed to the ability of these agents to cause DNA damage that results in the increased accumulation of mutations far

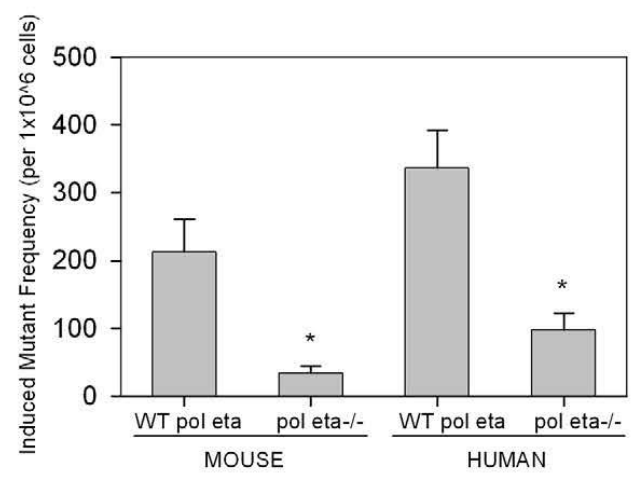

Figure 2. Induced mutant frequency is reduced in polymerase eta-deficient cells after BPDE treatment. Mutant frequency induced by $150 \mathrm{nM}$ BPDE was calculated relative to the frequency of vehicle treated cells and expressed as average \pm SEM. ${ }^{*} p<0.05$. doi:10.1371/journal.pone.0039596.g002 
Table 1. Kinds of Mutations Induced in the Hprt Gene of Murine Dermal Fibroblasts.

\begin{tabular}{|c|c|c|c|c|}
\hline & \multicolumn{2}{|c|}{ Pol eta+/+ } & \multicolumn{2}{|c|}{ Pol eta-/- } \\
\hline & No. & $\%$ & No. & $\%$ \\
\hline Deletion of one Purine & 1 & 4.55 & 1 & 4.35 \\
\hline Tandems & 2 & 9.09 & 0 & - \\
\hline \multicolumn{5}{|l|}{ Transitions } \\
\hline $\mathrm{G} \cdot \mathrm{C} \rightarrow \mathrm{A} \cdot \mathrm{T}$ & 4 & 18.18 & 3 & 13.04 \\
\hline $\mathrm{A} \cdot \mathrm{T} \rightarrow \mathrm{G} \cdot \mathrm{C}$ & 1 & 4.55 & 2 & 8.70 \\
\hline \multicolumn{5}{|l|}{ Transversions } \\
\hline $\mathrm{G} \cdot \mathrm{C} \rightarrow \mathrm{T} \cdot \mathrm{A}$ & 11 & 50.00 & 16 & 69.57 \\
\hline $\mathrm{G} \cdot \mathrm{C} \rightarrow \mathrm{C} \cdot \mathrm{G}$ & 0 & - & 1 & 4.35 \\
\hline$A \cdot T \rightarrow T \cdot A$ & 1 & 4.55 & 0 & - \\
\hline$A \cdot T \rightarrow C \cdot G$ & 2 & 9.09 & 0 & - \\
\hline Total & 22 & 100 & 23 & 100 \\
\hline
\end{tabular}

doi:10.1371/journal.pone.0039596.t001

exceeding those that occur spontaneously. The ability of a cell to bypass DNA-damage during replication prevents prolonged stalling of replication fork complexes that could signal cell death. However, depending on both the lesion encountered and the polymerase recruited, this bypass could result in replication errors that become mutations upon subsequent cell divisions. Polymerases of the Y-family are well known to participate in bypass replication and research has been focused on determining the accuracy of each enzyme when a particular DNA lesion is encountered. While polymerase kappa is likely specialized for protection against endogenously produced DNA adducts including those resulting from reactive oxygen species, this enzyme has also been implicated in the error-free bypass of BPDE lesions [ $\left.\begin{array}{ll}13 & 15\end{array}\right]$. Other members of this polymerase family however, namely pol eta and pol iota, appear to have lower fidelity when they encounter BPDE-adducted bases. It was previously reported that pol eta may be involved in error-prone bypass of BPDE adducts using in vitro and yeast systems $[18,22,27]$. The only data examining pol eta in mammalian cells showed that human fibroblasts without a functional copy of pol eta were equally mutable relative to their wild-type counterparts after exposure to BPDE, indicating that pol eta does not participate in BPDE adduct bypass [20]. However, in this investigation, the eta-null XP variant cells and the normal human fibroblasts were not treated simultaneously, rather the normal fibroblast results were obtained from a different study in which a modified treatment protocol was used [28]. In the current study both murine and human fibroblasts without a functional pol eta accumulated fewer BPDE-induced mutations indicating an error-prone role for this enzyme in the bypass of BPDE-adducted DNA. Additionally, the current study used an asynchronous population of cells to determine mutant frequency while the previous studies used synchronized cells. Another possible reason for discrepancies in the induced mutant frequency in pol etadeficient human cells between these two studies may be due to differences in the cell lines used as they were derived from different XP variant patients. XP4BE cells used by Watanabe et al [20] express a truncated version of pol eta that is 27 amino acids long due to a deletion of four nucleotides in the gene, while the XP115LO cells used in this study express a 127 amino acid truncated form as a result of a nonsense mutation. However, neither enzyme has been shown to have measurable enzyme activity. Data presented here from mouse cells support the low mutant frequency seen in human XP variant cells in a second species. In addition, pol eta was disrupted in these mice by removal of exon 4 and the generation of a frameshift mutation $30 \mathrm{bp}$ downstream. The resultant mutant transcript would therefore encode a 92 amino acid truncated form of pol eta. This deletion is similar to that found in XP4BE cells and supports our hypothesis that loss of functional pol eta reduces BPDE-induced mutagenesis.

The observation of a reduced mutant frequency in pol eta-null mouse fibroblasts and in human fibroblasts deficient for pol eta relative to their pol eta wild-type counterparts in response to BPDE exposure indicates that this enzyme is involved in errorprone bypass of BPDE-adducts, in contrast to its error-free role in the bypass of UV-induced lesions. Thus, while it would be catastrophic to target pol eta in the body as a whole as an anticancer strategy, increasing the risk of UV-induced mutations and cancers, it may be possible to selectively inhibit this enzyme in tissues other than the skin that accumulate bulky lesions like those induced by BPDE. It was recently shown that ribozyme-mediated knockdown of the Y-family polymerase REVl in the lung via aerosol delivery reduced the multiplicity of lung tumors in $\mathrm{B}[\mathrm{a}] \mathrm{P}-$ treated mice [29]. Lung-directed targeting of pol eta may be useful for those with increased exposure to environmental BPDE, as is the case for smokers. Determining the role of pol eta in the bypass of BPDE-adducts will further our knowledge of environmental carcinogen-linked cancers with the intent to determine possible prevention strategies.

\section{Materials and Methods}

\section{Ethics Statement}

This study was carried out in strict accordance with the recommendations in the Guide for the Care and Use of Laboratory Animals of the National Institutes of Health. The protocol was approved by the Institutional Animal Care and Use Committee at the University of Louisville protocol number 09059. All efforts were made to minimize animal suffering.

\section{Cells and Cell Culture}

Pol eta knockout mice were a gift from the Kucherlapati laboratory [30]. These mice were backcrossed with G57BL/ 6 mice and were congenic in the $\mathrm{C} 57 \mathrm{BL} / 6$ genetic background. Mice were tested for mutations in pol eta and pol iota using PCR-based protocols $[31,32]$. All mice used were proficient for pol iota. Primary murine fibroblasts were established from 8- to 9-week-old mice using standard techniques as published [33]. Briefly, using an autoclaved ear punch, small pieces of ear from anesthetized mice were removed and plated in MEM-a (Life Technologies) supplemented with $10 \%$ fetal calf serum (Hyclone), $2 \mathrm{mmol} / \mathrm{L}$ glutamine, nonessential amino acids (Life Technologies), penicillin (100 units $/ \mathrm{mL}$ ), streptomycin $(100 \mathrm{ug} / \mathrm{mL})$, and fungizone (Life Technologies; $1 \mathrm{ug} / \mathrm{mL}$ ). Fibroblasts were grown at $37^{\circ} \mathrm{C}$ under hypoxic conditions (2 $3 \% \mathrm{O} 2,5 \% \mathrm{CO} 2$ ) previously reported to increase the number of population doublings and lengthen the time before senescence of primary murine cells [34]. The medium was changed within $48 \mathrm{~h}$, and the fungizone was omitted.

NF 1604 human lung embryonic fibroblasts human fibroblasts were a gift from Dr. Lisa McDaniels [35] at the University of Texas Southwestern Medical Branch under the terms of an MTA between Greon Corporation and WGM. XP115LO fibroblasts were a gift from Dr. Veronica Maher [36]. Human fibroblasts were grown in DMEM (Gibco) supplemented with $10 \%$ supple- 
mented calf serum (Atlanta Biologicals), penicillin (100 units $/ \mathrm{mL}$ ), and streptomycin $\left(100 \mathrm{ug} / \mathrm{mL}\right.$ ) (Sigma) at $37^{\circ} \mathrm{C}$ in $5 \% \mathrm{CO}_{2}$.

\section{Exposure to BPDE}

BPDE was purchased from the National Cancer Institute Chemical Carcinogen Repository. Powdered BPDE was dissolved in anhydrous tetrahydrofuran (THF) (Sigma). For cytotoxic and mutagenic studies the culture medium was aspirated and the cells were washed with sterile PBS (pH 7.4) and replaced with serumfree medium. BPDE was added to the culture medium to a final concentration of $150 \mathrm{nM}$. The control cells were exposed to solvent (THF) only. After 1 hour incubation at $37^{\circ} \mathrm{C}$, the cells were washed twice with PBS and the medium was replaced with complete medium.

\section{Cytotoxic and Mutagenic Assays}

Primary mouse fibroblasts were assayed for cytotoxic and mutagenic responses to BPDE at early passages (six to eight population doublings) after primary cultures were established. A series of independent populations $\left(1.5 \times 10^{6}\right.$ cells each, plated on 150-mm-diameter plates) were allowed to attach overnight before exposure to BPDE. Cytotoxic responses to BPDE were established by exposing the cells at the same density as used for the mutagenesis experiments, namely $1 \times 10^{4}$ cells $/ \mathrm{cm}^{2}$. After exposure, the cells were plated at cloning density to measure colony-

\section{References}

1. Cosman M, de los Santos C, Fiala R, Hingerty BE, Singh SB, et al. (1992) Solution conformation of the major adduct between the carcinogen $(+)$-antibenzo[a]pyrene diol epoxide and DNA. Proc Nat Acad Sci U S A 89: 1914 benzo[
1918.

2. Yang LL, Maher VM, McCormick J (1980) Error-free excision of the cytotoxic, mutagenic N2-deoxyguanosine DNA adduct formed in human fibroblasts by ( + ) -)-7 beta, 8 alpha-dihydroxy- 9 alpha, 10 alpha-epoxy-7,8,9,10-tetrahydrobenzo[a]pyrene. Proc Natl Acad Sci U S A 77: 59335937.

3. Dreij K, Seidel A, Jernstrom B (2005) Differential removal of DNA adducts derived from anti-diol epoxides of dibenzo[a, 1]pyrene and benzo[a]pyrene in human cells. Chem Res Toxicol 18: 655664 .

4. Ohmori H, Friedberg EG, Fuchs RP, Goodman MF, Hanaoka F, et al. (2001) The Y-family of DNA polymerases. Mol Cell 8: 78 .

5. Johnson RE, Kondratick CM, Prakash S, Prakash L (1999) hRAD30 mutations in the variant form of xeroderma pigmentosum. Science 285: 263265.

6. Broughton BG, Cordonnier A, Kleijer WJ, Jaspers NG, Fawcett H, et al. (2002) Molecular analysis of mutations in DNA polymerase eta in xeroderma pigmentosum-variant patients. Proc Natl Acad Sci U S A 99: 815820.

7. Tung BS, McGregor WG, Wang YG, Maher VM, McCiormick JJ (1996) Comparison of the rate of excision of major UV photoproducts in the strands of the human HPRT gene of normal and xeroderma pigmentosum variant cells. Mutat Res 362: 65 74 .

8. Maher VM, Ouellette LM, Gurren RD, McGormick JJ (1976) Frequency of ultraviolet light-induced mutations is higher in xeroderma pigmentosum variant cells than in normal human cells. Nature 261: 593595.

9. Washington MT, Johnson RE, Prakash L, Prakash S (2001) Accuracy of lesion bypass by yeast and human DNA polymerase eta. Proc Natl Acad Sci U S A 98 : 83558360 .

10. Dumstorf CA, Clark AB, Lin Q, Kissling GE, Yuan T, et al. (2006) Participation of mouse DNA polymerase iota in strand-biased mutagenic bypass of UV photoproducts and suppression of skin cancer. Proc Natl Acad Sci U S A 103 1808318088.

11. Gueranger Q, Stary A, Aoufouchi S, Faili A, Sarasin A, et al. (2008) Role of DNA polymerases eta, iota and zeta in UV resistance and UV-induced mutagenesis in a human cell line. DNA Repair (Amst) 7: 15511562.

12. Wang Y, Woodgate R, McManus TP, Mead S, McCormick JJ, et al. (2007) Evidence that in xeroderma pigmentosum variant cells, which lack DNA polymerase eta, DNA polymerase iota causes the very high frequency and unique spectrum of UV-induced mutations. Cancer Res 67: 30183026.

13. Zhang $Y$, Wu $X$, Guo D, Rechkoblit O, Wang $Z$ (2002) Activities of human DNA polymerase kappa in response to the major benzo[a]pyrene DNA adduct: error-free lesion bypass and extension synthesis from opposite the lesion. DNA Repair (Amst) 1: 559569.

14. Ogi T, Mimura J, Hikida M, Fujimoto H, Fujii-Kuriyama Y, et al. (2001) Expression of human and mouse genes encoding polkappa: testis-specific
developmental regulation and AhR-dependent inducible transcription. Genes developmental reg
Cells 6: 943953 . forming ability. Fresh media was added 7 days after treatment and cells were stained with $1 \%$ crystal violet (Fisher Scientific) at 14 days. For mutagenesis, cells were allowed 35 days of growth before trypsinization and passage of $1.5 \times 10^{6}$ cells. After 89 days of post-treatment growth, cells were selected in $20 \mathrm{uM} 6$ thioguanine (TG) at a density of 450 cells $/ \mathrm{cm}^{2}$. Colony-forming ability was also determined at the time of TG selection by plating the cells at cloning density in nonselective medium and was used to correct for the mutant frequency. After 1420 days, TG resistant clones were isolated for Hprt coding region amplification. After colony isolation, plates were stained, and mutant frequency was determined, defined as the number of observed TG clones per $10^{6}$ clonable cells (corrected for cloning efficiency). Data were analyzed using a student's t-test assuming unequal variance.

Amplification and Sequencing of $\boldsymbol{H p r t}$ cDNA. Isolation of thioguanine clones, reverse transcriptase-PCR of the Hprt cDNA, and sequence analysis of the PCR products were performed as previously described $[10,33]$.

\section{Author Contributions}

Conceived and designed the experiments: ACK LJS WGM. Performed the experiments: ACK LJS TJB RLS. Analyzed the data: ACK LJS TJB RLS WGM. Contributed reagents/materials/analysis tools: TJB WGM. Wrote the paper: ACK LJS.

15. Avkin S, Goldsmith M, Velasco-Miguel S, Geacintov N, Friedberg EG, et al. (2004) Quantitative analysis of translesion DNA synthesis across a benzo[a]pyrene-guanine adduct in mammalian cells: the role of DNA polymerase kappa. J Biol Chem 279: 5329853305.

16. Ogi T, Shinkai Y, Tanaka K, Ohmori H (2002) Polkappa protects mammalian cells against the lethal and mutagenic effects of benzo[a]pyrene. Proc Natl Acad Sci U S A 99: 1554815553

17. Rechkoblit $\mathrm{O}$, Zhang $\mathrm{Y}$, Guo D, Wang Z, Amin S, et al. (2002) trans-Lesion synthesis past bulky benzo[a]pyrene diol epoxide N2-dG and N6-dA lesions catalyzed by DNA bypass polymerases. J Biol Chem 277: 3048830494 .

18. Chiapperino D, Kroth H, Kramarczuk IH, Sayer JM, Masutani C, et al. (2002) Preferential misincorporation of purine nucleotides by human DNA polymerase eta opposite benzo[a]pyrene 7,8-diol 9,10-epoxide deoxyguanosine adducts. J Biol Chem 277: 1176511771.

19. Shachar S, Ziv O, Avkin S, Adar S, Wittschieben J, et al. (2009) Twopolymerase mechanisms dictate error-free and error-prone translesion DNA polymerase mechanisms dictate error-free and
synthesis in mammals. EMBO J 28: $383 \quad 393$.

20. Watanabe M, Maher VM, McCormick JJ (1985) Excision repair of UV- or benzo[a] pyrene diol epoxide-induced lesions in xeroderma pigmentosum variant cells is 'error free'. Mutat Res 146: 285294.

21. Simhadri S, Kramata P, Zajc B, Sayer JM, Jerina DM, et al. (2002) Benzo[a]pyrene diol epoxide-deoxyguanosine adducts are accurately bypassed by yeast DNA polymerase zeta in vitro. Mutat Res 508: 137145.

22. Xie Z, Braithwaite E, Guo D, Zhao B, Geacintov NE, et al. (2003) Mutagenesis of benzo[a]pyrene diol epoxide in yeast: requirement for DNA polymerase zeta and involvement of DNA polymerase eta. Biochemistry 42: 1125311262.

23. Zhao B, Wang J, Geacintov NE, Wang Z (2006) Poleta, Polzeta and Rev1 together are required for $\mathrm{G}$ to $\mathrm{T}$ transversion mutations induced by the $(+)$ - and (-)-trans-anti-BPDE-N2-dG DNA adducts in yeast cells. Nucleic Acids Res 34: 417425 .

24. Neal JA, Fletcher KL, McCormick JJ, Maher VM (2010) The role of hRev7, the accessory subunit of $h$ Polzeta, in translesion synthesis past DNA damage induced accessory subunit of hPolzeta, in translesion synthesis past DNA da
by benzo[a]pyrene diol epoxide (BPDE). BMC Cell Biol 11: 97.

25. Hashimoto K, Cho Y, Yang IY, Akagi J, Ohashi E, et al. (2012) The Vital Role of Polymerase $\zeta$ and REV1 in Mutagenic, but Not Correct, DNA Synthesis across Benzo[a]pyrene-dG and Recruitment of Polymerase $\zeta$ by REV1 to Replication-stalled Site. J Biol Chem 287: 96139622.

26. Crow JF (1995) Spontaneous mutation as a risk factor. Exp Clin Immunogenet 12: 121128.

27. Chiapperino D, Ciai M, Sayer JM, Yagi H, Kroth H, et al. (2005) Error-prone translesion synthesis by human DNA polymerase eta on DNA-containing deoxyadenosine adducts of 7,8-dihydroxy-9,10-epoxy-7,8,9,10-tetrahydrobenzo[a]pyrene. J Biol Chem 280: 3968439692.

28. Yang LL, Maher VM, McCormick JJ (1982) Relationship between excision repair and the cytotoxic and mutagenic effect of the 'anti' 7,8-diol-9,10-epoxide of benzo[a]pyrene in human cells. Mutat Res 94: 435447. 
29. Dumstorf CA, Mukhopadhyay S, Krishnan E, Haribabu B, McGregor WG (2009) REV1 is implicated in the development of carcinogen-induced lung cancer. Mol Ciancer Res 7: 247254

30. Lin Q, Clark AB, McCulloch SD, Yuan T, Bronson RT, et al. (2006) Increased susceptibility to UV-induced skin carcinogenesis in polymerase eta-deficient mice. Ciancer Res 66: 8794

31. Lin Q, Clark AB, McCulloch SD, Yuan T, Bronson RT, et al. (2006) Increased Susceptibility to UV-Induced Skin Carcinogenesis in Polymerase \{eta\}-deficien Mice. Ciancer Research 66: 8794.

32. McDonald JP, Frank EG, Plosky BS, Rogozin IB, Masutani C, et al. (2003) 129 derived strains of mice are deficient in DNA polymerase iota and have normal immunoglobulin hypermutation. The Journal of Experimental Medicine 198: 635643 .

33. Diaz M, Watson NB, Turkington G, Verkoczy LK, Klinman NR, et al. (2003) Decreased frequency and highly aberrant spectrum of ultraviolet-induced mutations in the hprt gene of mouse fibroblasts expressing antisense RNA to DNA polymerase zeta. Mol Cancer Res 1: 836847.

34. Parrinello S, Samper E, Krtolica A, Goldstein J, Melov S, et al. (2003) Oxygen sensitivity severely limits the replicative lifespan of murine fibroblasts. Nat Gell Biol 5: 741747 .

35. Ouellette MM, McDaniel LD, Wright WE, Shay JW, Schultz RA (2000) The chrablishment of telomerase-inmes. Human molecular genetics 9. 403411.

36. Wang Y, Woodgate R, McManus TP, Mead S, McCormick JJ, et al. (2007) Evidence that in Xeroderma Pigmentosum Variant Gells, which Lack DNA Polymerase \{eta\}, DNA Polymerase \{iota\} Causes the Very High Frequency and Unique Spectrum of UV-Induced Mutations. Cancer Research 67: 3018 3026. 


\section{CURRICULUM VITAE}

Name: $\quad$ Alden Klarer

Education/Training:

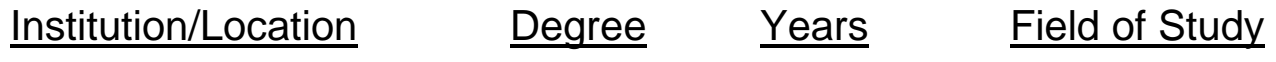

University of Kentucky $\quad$ B.S. 2000-2004 Biological Sciences

University of Louisville - 2007-2009 Medicine

University of Louisville M.S. 2009-2011 Biochemistry and Molecular Biology

Research and Job Experience:

\begin{tabular}{|c|c|c|}
\hline Position/Title & Year & Employer/Location \\
\hline Student Laboratory Technician & $2002-2004$ & $\begin{array}{l}\text { University of Kentucky, } \\
\text { College of Agriculture }\end{array}$ \\
\hline Laboratory Technician III & 2004-2007 & $\begin{array}{l}\text { University of Louisville, } \\
\text { Department of Surgery }\end{array}$ \\
\hline $\begin{array}{l}\text { Graduate Research Assistant } \\
\text { Lexington, KY }\end{array}$ & 2009-Present & $\begin{array}{l}\text { University of Louisville, } \\
\text { Department of } \\
\text { Biochemistry and } \\
\text { Molecular Biology }\end{array}$ \\
\hline
\end{tabular}




\section{Awards/Honors:}

2009-present: NIEHS, Ruth L. Kirschstein National Research Service Awards for Individual Predoctoral MD/PhD and Other Dual Doctoral Degree Fellows F30). Award amount: \$153,451. Start date 07-01-2009, end date 06/30/2015.

2007-present: M.D./Ph.D. Program, University of Louisville School of Medicine, full tuition scholarship and stipend during Medical School and Graduate School.

2000-2004: Academic Scholarships: (i) University of Kentucky Bluegrass Scholarship; (ii) University of Kentucky Valedictorian Scholarship; and (iii) Kentucky Educational Excellence Scholarship (Commonwealth of Kentucky).

2000-2004: University of Kentucky Honors Program

2000-2004: Deans List, University of Kentucky

2003: Beta Beta Beta Biological Honors Society, in recognition of academic scholarship in Biology.

\section{Publications:}

Clem BF, O'Neal J, Tapolsky G et al. Targeting 6-Phosphofructo-2-Kinase (PFKFB3) as a Therapeutic Strategy against Cancer. Mol Cancer Ther 2013.

Klarer AC, Stallons LJ, Burke TJ et al. DNA Polymerase Eta Participates in the Mutagenic Bypass of Adducts Induced by Benzo[a]pyrene Diol Epoxide in Mammalian Cells. PLoS One 2012; 7:e39596.

Telang S, Nelson KK, Siow DL et al. Cytochrome c oxidase is activated by the oncoprotein Ras and is required for A549 lung adenocarcinoma growth. Mol Cancer 2012; 11:60.

Telang S, Clem BF, Klarer AC et al. Small Molecule Inhibition of 6Phosphofructo-2-Kinase Suppresses T Cell Activation. J Transl Med 2012; 10:95

Telang S, Rasku MA, Clem AL et al. Phase II trial of the regulatory $\mathbf{T}$ celldepleting agent, denileukin diftitox, in patients with unresectable stage IV melanoma. BMC Cancer 2011; 11:515. 
T. R. Stefaniak, D. L. Hyten, V. R. Pantalone, A. Klarer, and T. W. Pfeiffer. Soybean Cultivars Resulted from More Recombination Events Than Unselected Lines in the Same Population. Crop Science 2006 46: 43-51.

\section{Presentations:}

Biochemistry and Molecular Biology Seminar Series. December 14, 2009. Homberger Library. "The Nucleoside Analogue, 8-Aminoadenosine, has a Novel Metabolic Mechanism in Multiple Myeloma."

MD/PhD Monthly Seminar Series. November 17 $17^{\text {th }}$ 2010. "Effect of Environmental Insults on Tumor Initiation and Progression".

Biochemistry and Molecular Biology Seminar Series. May 9, 2011. Homberger Library. "Activated Ras requires autophagy to maintain oxidative metabolism and tumorigenesis".

Biochemistry and Molecular Biology, EXAM IIA. February $1^{\text {st }}$, 2012. Homberger Library.

Biochemistry and Molecular Biology PhD Defense, May 21 ${ }^{\text {st }}$, 2013. Homberger Library. "6-Phosphofructo-2-Kinase Inhibition Induces Autophagy as a Survival Mechanism".

\section{Poster presentations:}

Research Louisville 2006. "Clustering of Papillary Thyroid Carcinomas from African-American and Caucasian Female Subjects into Distinctly Different Microarray Expression Profiles". A. Klarer, R. Goldstein. University of Louisville Department of Surgery.

J. Graham Brown Cancer Center Annual Retreat 2007 and Research!Louisville Conference 2007. "Targeting Transaldolase In Cancer". Klarer, A., Nelson, K., Clem, A., Yalcin, A., Telang, S., Clem, B., Thornburg, J., Rasku, M., and Chesney, J. Molecular Targets Group, Brown Cancer Center, University of Louisville

Research!Louisville Conference 2008 and J. Graham Brown Cancer Center Annual Retreat 2008. "DNA polymerase eta is error-prone when bypassing adducts induced in DNA by benzo[a]pyrene diol epoxide". Alden C. Klarer ${ }^{1}$, Tom Burke ${ }^{1}$, and W. Glenn McGregor ${ }^{1,2}{ }^{1}$ Department of Pharmacology and Toxicology, ${ }^{2}$ Molecular Targets Program, ${ }^{2}$ Cancer Prevention and Control Program, J.G Brown Cancer Center University of Louisville School of Medicine 
2010 Keystone Symposia Cell Death Pathways: Apoptosis, Autophagy and Necrosis and Metabolism and Cancer Progression March 12-17, 2010. "Role of DNA polymerases eta and iota in mutagenic translesion synthesis and a potential suppressor function against skin cancer". A Klarer ${ }^{1}$, LJ Stallons ${ }^{2}$, and W.G. McGregor ${ }^{2}$. ${ }^{1}$ Department of Biochemistry, ${ }^{2}$ Department of Pharmacology and Toxicology, University of Louisville.

Lung Cancer Symposium at the University of Kentucky, 3-27-2010. "Role of DNA polymerases eta and iota in mutagenic translesion synthesis and a potential suppressor function against skin cancer". A Klarer ${ }^{1}$, LJ Stallons ${ }^{2}$, and W.G. McGregor ${ }^{2}$. 'Department of Biochemistry, ${ }^{2}$ Department of Pharmacology and Toxicology, University of Louisville.

Southern Regional Meeting, New Orleans, Louisiana, February 25-27 ${ }^{\text {th }}, 2010$. "Role of DNA polymerases eta and iota in mutagenic translesion synthesis and a potential suppressor function against skin cancer". A Klarer ${ }^{1}$, LJ Stallons ${ }^{2}$, and W.G. McGregor ${ }^{2}$. ${ }^{1}$ Department of Biochemistry, ${ }^{2}$ Department of Pharmacology and Toxicology, University of Louisville.

J. Graham Brown Cancer Center Retreat, November 5 ${ }^{\text {th }}, 2010$ and Research!Louisville Conference October 11-15 ${ }^{\text {th }}$ 2010. "PFKFB3 is required for resistance to apoptosis under hypoxia". Alden Klarer ${ }^{1}$, Kavitha Yaddanapudi ${ }^{1}$, William G. McGregor ${ }^{2}$, Sucheta Telang ${ }^{1}$. University of Louisville, Department of Biochemistry and Molecular Biology ${ }^{1}$, Department of Pharmacology and Toxicology 2 .

University of Louisville, Department of Biochemistry and Molecular Biology Program Retreat, August $26^{\text {th }}, 2011$ and Research!Louisville Conference October $11^{\text {th }}, 2011$. "Starvation induced by glycolytic inhibition promotes autophagy in neoplastic cells". A Klarer ${ }^{1}$, W McGregor ${ }^{2}$, S Ellis ${ }^{1}$, S Telang ${ }^{1}$ University of Louisville, Department of Biochemistry and Molecular Biology ${ }^{1}$, Department of Pharmacology and Toxicology ${ }^{2}$.

South-Central Regional MD/PhD Conference, September $8^{\text {th }}, 2012$. "Inhibition of 6-phosphofructo-2-kinase (PFKFB3) induces autophagy in neoplastic cells". A Klarer ${ }^{1}$ S Ellis ${ }^{1}$, S Telang ${ }^{1}$ University of Louisville, Department of Biochemistry and Molecular Biology ${ }^{1}$ 